Universidade de São Paulo

Instituto de Física

\title{
Quantização Covariante de Sistemas Mecânicos
}

\author{
João Luis Meloni Assirati
}

Orientador: Prof. Dr. Dmitri Maximovitch Guitman

Tese de doutorado apresentada ao

Instituto de Física para a obtenção

do título de Doutor em Ciências.

Banca Examinadora

Prof. Dr. Dmitri Maximovitch Guitman (IF-USP)

Prof. Dr. Josif Frenkel (IF-USP)

Prof. Dr. Oscar José Pinto Éboli (IFUSP)

Prof. Dr. Andrei Bytsenko (UEL)

Prof. Dr. Jeferson de Lima Tomazelli (UFSC)

São Paulo 


\section{FICHA CATALOGRÁFICA}

\section{Preparada pelo Serviço de Biblioteca e Informação}

do Instituto de Física da Universidade de São Paulo

Assirati, João Luis Meloni

Quantização covariante de sistemas mecânicos. - São Paulo, 2010.

Tese (Doutorado) - Universidade de São Paulo. Instituto de Física, Depto. de Física Nuclear

Orientador: Prof. Dr. Dmitri Maximovitch Guitman

Área de Concentração: Física

Unitermos: 1. Sistema quântico; 2.Teoria quântica relativística; 3. Física teórica; 4. Relatividade (Física); 5. Física matemática. 


\section{Resumo}

Estudamos as restrições impostas pelo princípio da covariância sobre o procedimento de quantização em espaços planos e curvos. Mostramos que o conjunto de todas as quantizações covariantes em espaços planos em coordenadas retangulares é composto de ordenamentos de operadores de posição e momento e exibimos uma parametrização funcional deste conjunto. Deduzimos regras para a quantização covariante em espaços planos em coordenadas gerais. Generalizamos estas quantizações para espaços curvos e mostramos que nestes espaços, além da ambiguidade de ordenamento, surge uma nova ambiguidade relacionada à curvatura. Este novo tipo de ambiguidade explica o surgimento de uma classe grande de "potenciais quânticos" no problema da quantização de uma partícula não relativística em um espaço curvo. 


\begin{abstract}
We study the restrictions imposed by the covariance principle on the quantization procedure in flat and curved spaces. We show that the set of all covariant quantizations in flat spaces in rectangular coordinates is composed of position and momentum operator orderings and exhibit a functional parametrization of this set. We deduce rules for the covariant quantization in flat spaces in general coordinates. We generalize these quantizations for curved spaces and show that in such spaces, besides the ordering ambiguity, it appears a new ambiguity related to the curvature. This new kind of ambiguity explains the appearence of a wide class of "quantum potentials" in the problem of quantization of a non-relativistic particle in curved space.
\end{abstract}


Agradeço aos que tornaram possível a existência deste trabalho:

Ao meu orientador, Dmitri Maximovitch Gitman.

Ao Conselho Nacional de Desenvolvimento Científico e Tecnológico - CNPq.

Aos meus pais, Emílio e Catarina.

Aos professores Josif Frenkel, Oscar José Pinto Éboli, Andrei Bytsenko e Jeferson de Lima Tomazelli, membros da banca examinadora.

Ao Instituto de Física da USP e seus funcionários.

Aos meus amigos, especialmente Rodrigo Fresneda e Suiane Inês da Costa Fernandes, Mário César Baldiotti, Arnaldo Gomes de Oliveira, Márcio Pinheiro Beck Eichler e Rosângela Aparecida Santos Eichler, Ivan Yasuda, Thiago dos Santos Pereira, Milton Alexandre da Silva Júnior e Ronaldo Carlotto Batista. 


\section{Sumário}

1 Introdução 3

1.1 Definição geral de quantização . . . . . . . . . . . . . . . . . 5

1.2 Quantização e covariância . . . . . . . . . . . . . . 6

2 Ordenamento de operadores no espaço plano $\quad 9$

2.1 Algumas propriedades razoáveis para quantizações . . . . . . . . . . 10

2.2 Covariância em espaços euclidianos ou pseudo-euclidianos . . . . . . . 13

2.3 Exemplos de ordenamentos em espaços euclidianos ou pseudo-euclidianos. 18

2.4 Símbolos de operadores e limite clássico em espaços euclidianos ou pseudo-euclidianos . . . . . . . . . . . . . . . . 21

2.5 Uma forma alternativa para $Q^{\theta}$ e $Q_{\omega} \ldots \ldots . \ldots . . \ldots . . \ldots 23$

2.6 Quantização em coordenadas gerais . . . . . . . . . . . . 26

2.7 Representação da posição em coordenadas gerais e exemplos de operadores . . . . . . . . . . . . . . . . . . . 32

3 Generalização de $Q_{\omega}$ a espaços curvos 36

3.1 Primeira generalização de $Q_{\omega}$ em espaços curvos . . . . . . . . . . . 36

3.2 Covariância de $Q_{\omega, 1}$ em espaços curvos . . . . . . . . . . . . . . . . . 40

3.3 Elemento de matriz de $Q_{\omega, 1}(F) \ldots \ldots$. . . . . . . . . . . . . . 42

$3.4 \omega, 1$-símbolos . . . . . . . . . . . . . . . . 47

3.5 Propriedades da função $\mathcal{M}(x, \xi) \ldots \ldots . \ldots$. . . . . . . . . . . . . . . . . . . 51

3.6 Forma geral de $Q_{\omega}$ em espaços curvos . . . . . . . . . . . . . 56

3.7 Covariância de $Q_{\omega, \mathcal{F}} \quad \ldots \ldots \ldots$. . . . . . . . . . . . . . . 58

3.8 Produto de símbolos e limite clássico . . . . . . . . . . . . . . . . 60

3.9 Quantização da partícula livre em um espaço curvo . . . . . . . . . . 67

$\begin{array}{ll}\text { A Propriedades do operador } e^{i(\eta \hat{x}+\hat{p} \xi)} & \mathbf{7 0}\end{array}$

B Definições e fórmulas da geometria riemanniana $\quad 72$ 
C A função exponencial $\quad \mathbf{7 5}$

C.1 Definição e propriedades da função exponencial . . . . . . . . . . . . 75

C.2 Coordenadas normais e geodésica mínima . . . . . . . . . . . . . . 80

C.3 Determinante jacobiano do fluxo geodésico . . . . . . . . . . . . . 82

D Uma fórmula para a derivada do produto 84 


\section{Capítulo 1}

\section{Introdução}

O problema geral da quantização de um sistema físico clássico em um espaço de coordenadas $(x, p)$, que consiste em associar operadores em um espaço de Hilbert adequado a funções do espaço de fase $F(x, p)$ (ver a seção 1.1) define um conjunto mínimo de propriedades cujo objetivo é assegurar que no limite clássico (quando o parâmetro $\hbar \rightarrow 0$ ) o sistema quantizado corresponda ao sistema clássico original. Havendo uma "perda de detalhamento" no processo limite $\hbar \rightarrow 0$, pode-se apenas esperar que o processo de quantização não seja unívoco mas, antes, a um dado sistema clássico correspondam muitos sistemas quânticos, todos consistentes com o mecanismo geral de quantização.

Esta ambigüidade se manifesta primariamente no bem conhecido problema de ordenamento de operadores na quantização canônica. De fato, se os operadores $\hat{x}$ e $\hat{p}$ obedecem às relações de comutação $(1.2)$, à função clássica $F(x, p)=x^{2} p^{2}$ poderiam corresponder vários operadores: $\hat{F}_{1}=\hat{x} \hat{p}^{2} \hat{x}, \hat{F}_{2}=\hat{p} \hat{x}^{2} \hat{p}, \hat{F}_{1}=\left(\hat{x}^{2} \hat{p}^{2}+\hat{p}^{2} \hat{x}^{2}\right) / 2$ etc. No entanto, tais ambuigüidades podem e devem ser resolvidas ou ao menos restringidas pela aplicação de outros princípios físicos. Neste trabalho, estudamos as restrições que o princípio de covariância impõe sobre as ambiguidades de ordenamento.

Começamos por definir o que significa covariância no contexto da quantização (seção 1.2). Definimos que sistemas quânticos derivados de um mesmo sistema clássico expresso em diferentes coordenadas (aqui, coordenadas sempre significarão as coordenadas $x$ espaciais, e não coordenadas do espaço de fase $(x, p)$ ) devem se relacionar por um isomorfismo isométrico $U$ que relaciona os espaços de Hilbert resultantes e, de forma consistente, os operadores. Desta maneira, operadores correspondentes à mesma função clássica em coordenadas diferentes possuirão o mesmo espectro. Esta discussão é fundamental para todo o trabalho e é importante, em particular, pelo fato de que é possível definir quantizações "ingênuas" para o problema da partícula 
livre não relativística em um espaço curvo (ou mesmo sem curvatura, mas em coordenadas gerais) que dependem do sistema de coordenadas usado [6]. Neste caso, obtém-se um espectro para o hamiltoniano que dependerá essencialmente do sistema de coordenadas usado.

Em seguida, aplicamos o conceito de quantização covariante a espaços planos em coordenadas retilíneas (espaços euclidianos ou pseudo-euclidianos). Nestes espaços, devido à simetria do grupo de rotações, covariância significa invariância de forma. Começamos por impor ao conceito geral de quantização algumas propriedades que permitem identificá-lo como um processo de ordenamento de operadores (seção 2.1). Com esta forma mais restrita, é possível aplicar o princípio de covariância obtendo uma forma parametrizada: as quantizações $Q_{\omega}$ estão em correspondência com uma medida complexa na reta real $\omega(\theta)$ (equação 2.26). Este resultado inclui o importante caso do ordenamento de Weyl, e explica e particulariza a forma (2.8) usada em [1]. Finalmente, apresentamos o resultado acima em coordenadas não retilíneas, com regras elementares, mas inéditas, de quantização em coordenadas não retilíneas (seção 2.6).

Em seguida, apresentamos uma generalização das quantizações $Q_{\omega}$ a espaços com curvatura. O trabalho até o momento focalizou-se no problema da "quantização canônica" de uma partícula em um espaço riemanniano sem propriedades topológicas especiais (essencialmente, um aberto do $\mathbb{R}^{D}$ com um tensor métrico). Esta generalização é feita em duas etapas. Primeiro, é definida a quantização $Q_{\omega, 1}$ (seção 3.1), que é formalmente idêntica à quantização em um espaço plano em coordenadas gerais. A regra resultante é a substituição das derivadas comuns por derivadas covariantes na expressão dos operadores em representação da posição. O cálculo do elemento de matriz de um operador segundo esta quantização revela uma função $\mathcal{M}(x, \xi)$ que é identicamente igual a 1 se o espaço não tem curvatura (mesmo que sejam usadas coordenadas gerais). Tal função revela uma ambiguidade de quantização extra quando estamos em um espaço curvo. Sua substituição, no processo de quantização, por outra função $\mathcal{F}(x, \xi)$ com as mesmas propriedades (seção 3.5) leva à definição de novas quantizações $Q_{\omega, \mathcal{F}}$ (seção 3.6), que incluem não só ambiguidades de ordenamento (no parâmetro $\omega$ ) como também relativas à curvatura (parâmetro $\mathcal{F}$ ). As relações entre todos os símbolos de operadores relacionados às quantizações $Q_{\omega, \mathcal{F}}$ são calculadas (generalizando os resultados de $[10,9]$ ) e o limite clássico é demonstrado (seção 3.8). A quantização $Q_{\omega, \mathcal{F}}$ inclui várias quantizações usadas em $[2,3,4,5]$, que em geral consideraram apenas o caso de Weyl $(\omega(\theta)=\delta(\theta))$.

Finalmente, este resultado é aplicado ao velho problema da partícula não relativística livre em um espaço curvo (3.9). Mostrou-se que tal sistema não tem 
ambiguidade de ordenamento (sua quantização é independente de $\omega$ ) mas depende da escolha de $\mathcal{F}$. De fato, sempre é possível escolher uma função $\mathcal{F}(x, \xi)$ de modo a obter um potencial quântico de forma geral $f(R)$ (onde $R(x)$ é o escalar de curvatura e $f(R) \rightarrow 0$ quando $R \rightarrow 0$ ). Em particular, o resultado de [7] e [8] é reproduzido se usamos $\mathcal{F}=\mathcal{M}$.

\subsection{Definição geral de quantização}

Nesta seção, será dada uma definição geral de quantização a título de referência para as seções que seguem.

Seja dado um sistema clássico descrito em um espaço de fase $2 D$ dimensional de variáveis $\left(x^{1}, x^{2}, \ldots x^{D}, p_{1}, p_{2}, \ldots p_{D}\right)=(x, p)$ sem vínculos, isto é, cuja dinâmica é dada em termos dos parênteses de Poisson

$$
\{F, G\}=\sum_{\mu=1}^{D}\left(\frac{\partial F}{\partial x^{\mu}} \frac{\partial G}{\partial p_{\mu}}-\frac{\partial F}{\partial p_{\mu}} \frac{\partial G}{\partial x^{\mu}}\right)
$$

Uma quantização $Q$ deste sistema é uma correspondência entre as funções clássicas complexas $F=F(x, p)$ e operadores $\hat{F}=Q(F)$ em um espaço de Hilbert $\mathcal{R}$. Esta correspondência deve obedecer a algumas propriedades, listadas a seguir.

1. $Q$ deve ser linear e injetiva, ou seja, $Q(\alpha F+\beta G)=\alpha Q(F)+\beta Q(G)$, onde $\alpha$ e $\beta$ são números complexos, e $F \neq G \Rightarrow Q(F) \neq Q(G)$.

2. Se $F(x, p)$ é uma função complexa, então $(Q(F))^{\dagger}=Q\left(F^{*}\right)$, onde ${ }^{*}$ denota o complexo conjugado. Esta condição implica que, se $F$ é real, então $(Q(F))^{\dagger}=$ $Q(F)$, ou seja, $Q(F)$ é um operador auto-adjunto ${ }^{1}$

3. A quantização da função constante igual a 1 deve ser o operador identidade $\hat{I}$, $Q(1)=\hat{I}$. A quantização das funções $x^{\mu}$ e $p_{\mu}$ devem ser operadores

$$
Q\left(x^{\mu}\right)=\hat{x}^{\mu} \text { e } Q\left(p_{\mu}\right)=\hat{p}_{\mu}
$$

(auto-adjuntos os, pela proprieade 2) que obedeçam às relações canônicas de comutação

$$
\left[\hat{x}^{\mu}, \hat{x}^{\nu}\right]=\left[\hat{p}_{\mu}, \hat{p}_{\nu}\right]=0,\left[\hat{x}^{\mu}, \hat{p}_{\nu}\right]=i \hbar \delta_{\nu}^{\mu}
$$

${ }^{1}$ A hipótese mais simples de que $(Q(F))^{\dagger}=Q(F)$ quando $F$ é real é equivalente à propriedade 2. De fato, usando esta hipótese mais simples e a propriedade 1 , seja $F=G+i H$ complexa, com $G$ e $H$ reais, então $(Q(F))^{\dagger}=(Q(G)+i Q(H))^{\dagger}=Q(G)-i Q(H)=Q\left(F^{*}\right)$ 
Além do mais, o espaço de Hilbert $\mathcal{R}$ deve ser irredutível em relação a estes operadores, ou seja, qualquer operador $\hat{O}$ que comute com todos eles, $\left[\hat{O}, \hat{x}^{\mu}\right]=\left[\hat{O}, \hat{p}_{\mu}\right]=0$, deve ser um múltiplo da identidade, $\hat{O}=\lambda \hat{I}$.

4. $Q$ deve ter limite clássico. Esta propriedade se expressa através do conceito de produto de símbolos de operadores, que definimos a seguir. Se $Q(F)=$ $\hat{F}$, dizemos que a função complexa $F$ é o símbolo do operador $\hat{F}$ segundo a quantização $Q$. Sejam $F$ e $G$ os símbolos dos operadores $\hat{F}$ e $\hat{G}$. Define-se o produto $F * G$ como sendo o símbolo do operador $\hat{F} \hat{G}$, ou seja, $Q(F * G)=\hat{F} \hat{G}$. Da mesma forma que o produto de operadores, o produto de símbolos é bilinear e associativo, mas em geral não comutativo. $Q$ tem limite clássico quando o seu produto de símbolos obedece às equações

$$
\begin{gathered}
\lim _{\hbar \rightarrow 0} F * G=F G \\
\lim _{\hbar \rightarrow 0} \frac{1}{i \hbar}(F * G-G * F)=\{F, G\}
\end{gathered}
$$

(o produto dos símbolos depende em geral da constante de Planck $\hbar)^{2}$

A propriedade 2 é indispensável do ponto de vista físico, já que um operador correspondente a uma função real deve ter espectro real. Por razões de conveniência matemática, podemos, no entanto, dispensá-la da definição de quantização. Quantizações que obedeçam a 2 serão chamadas de quantizações auto-adjuntas.

\subsection{Quantização e covariância}

Vamos considerar um sistema clássico com espaço configuracional $D$-dimensional de coordenadas $\left(x^{1}, x^{2}, \ldots, x^{D}\right)=x$ e lagrangiana não singular $L=L(x, \dot{x})$. A passagem ao formalismo canônico se dá pela introdução dos $D$ momentos

$$
p_{\mu}=\frac{\partial L}{\partial \dot{x}^{\mu}}(x, \dot{x})
$$

As equações de movimento são dadas pelo princípio variacional $\delta \int L d t=0$ e são, portanto, independentes do sistema de coordenadas $x$ do espaço de configurações,

\footnotetext{
${ }^{2}$ Usando (1.4), uma versão generalizada do "teorema de Ehrenfest" para qualquer observável $A$ é imediata:

$$
\left.\frac{d}{d t}\langle\hat{A}\rangle=\left\langle\frac{1}{i \hbar}[\hat{A}, \hat{H}]\right\rangle=\langle\widehat{A, H}\}\right\rangle+O(\hbar)
$$

onde $\hat{H}$ é o operador hamiltoniano, $A$ e $H$ são os símbolos de $\hat{A}$ e $\hat{H}$, e $\{$,$\} são os parênteses de$ Poisson.
} 
desde que a lagrangiana seja tratada como um escalar. Introduzindo novas coordenadas

$$
x^{\prime \mu}=\varphi^{\mu}(x),
$$

onde $\varphi$ é um difeomorfismo, temos (ver comentário sobre as derivadas parciais $\partial x / \partial x^{\prime}$ e $\partial x^{\prime} / \partial x$ no apêndice B) $\dot{x}^{\prime \mu}=\frac{\partial x^{\prime \mu}}{\partial x^{\alpha}} \dot{x}^{\alpha}$,

$$
L^{\prime}\left(x^{\prime}, \dot{x}^{\prime}\right)=L(x, \dot{x})=L\left(\varphi^{-1}\left(x^{\prime}\right), \frac{\partial x}{\partial x^{\prime}}\left(x^{\prime}\right) \dot{x}^{\prime}\right)
$$

e, portanto, as relações $\frac{\partial L^{\prime}}{\partial \dot{x}^{\prime \mu}}=\frac{\partial L}{\partial \dot{x}^{\alpha}} \frac{\partial x^{\alpha}}{\partial x^{\prime \mu}}$. Assim, os momentos nas novas coordenadas se expressam como

$$
p_{\mu}^{\prime}=p_{\alpha} \frac{\partial x^{\alpha}}{\partial x^{\prime \mu}}(x)
$$

As transformações (1.5) e (1.6) formam um sub-grupo de transformações canônicas independentes do tempo chamadas de transformações de ponto ${ }^{3}$. Sob estas transformações, as funções clássicas $A(x, p)$ se transformam como escalares, $A(x, p)=A^{\prime}\left(x^{\prime}, p^{\prime}\right)$, inclusive a função hamiltoniana, já que as transformações são independentes do tempo.

Naturalmente se coloca a questão de como definir quantizações de um sistema clássico expresso em um sistema de coordenadas arbitrário de maneira consistente. Isto significa que se $A(x, p)=A^{\prime}\left(x^{\prime}, p^{\prime}\right)$ é uma função clássica expressa em dois sistemas de coordenadas $x$ e $x^{\prime}$, então os operadores correspondentes $\hat{A}$ e $\hat{A}^{\prime}$ devem ter o mesmo espectro, em algum sentido. Vamos deixar esta afirmação mais precisa.

Suponhamos que para cada sistema de coordenadas $x, x^{\prime}, \ldots$ seja definida uma quantização $Q, Q^{\prime}, \ldots$ nos espaços de Hilbert $\mathcal{R}, \mathcal{R}^{\prime}, \ldots$, respectivamente. Se, para quaisquer duas tais quantizações $Q$ e $Q^{\prime}$ existe uma transformação linear inversível $U: \mathcal{R} \rightarrow \mathcal{R}^{\prime}$ tal que para qualquer função clássica $F(x, p)=F^{\prime}\left(x^{\prime}, p^{\prime}\right)$ os operadores $\hat{F}=Q(F)$ em $\mathcal{R}$ e $\hat{F}^{\prime}=Q^{\prime}\left(F^{\prime}\right)$ em $\mathcal{R}^{\prime}$ obedecem ${ }^{4}$

$$
Q^{\prime}\left(F^{\prime}\right)=U Q(F) U^{\dagger}
$$

então o conjunto destas quantizações $Q, Q^{\prime}, \ldots$ será chamada de quantização covariante. Podemos nos restringir a um determinado subgrupo de (1.5) e (1.6), dizendo que a quantização é covariante sob este determinado subgrupo.

\footnotetext{
${ }^{3} \mathrm{~A}$ transformações mais gerais do momento, canonicamente compatíveis com (1.5), são $p_{\mu}^{\prime}=\left(p_{\alpha}+\frac{\partial f}{\partial x^{\alpha}}(x)\right) \frac{\partial x^{\alpha}}{\partial x^{\prime \mu}}(x)$, onde $f=f(x)$, correspondente à transformação da lagrangiana $L^{\prime}\left(x^{\prime}, \dot{x}^{\prime}\right)=L(x, \dot{x})+\frac{d f}{d t}$. A teoria clássica resultante é idêntica, a não ser pelos valores do momento.

${ }^{4} \mathrm{~A}$ transformação adjunta $U^{\dagger}: \mathcal{R}^{\prime} \rightarrow \mathcal{R}$ é definida através do espaço dual $\mathcal{R}^{*} \operatorname{como}\langle\psi| U^{\dagger}=\left\langle\psi^{\prime}\right|$, sempre que $U|\psi\rangle=\left|\psi^{\prime}\right\rangle$.
} 
Tomando $F(x, p)=1 \mathrm{em}(1.7)$, vemos que $\hat{I}=U U^{\dagger}$ ou $U^{-1}=U^{\dagger}$, ou seja, $U$ é unitária. Se $Q(F)$ é um operador auto-adjunto, vemos que $Q^{\prime}\left(F^{\prime}\right)$ também o será, e se $Q(F)\left|\psi_{n}\right\rangle=\lambda_{n}\left|\psi_{n}\right\rangle$, então a equação (1.7) mostra que $Q^{\prime}\left(F^{\prime}\right)\left|\psi_{n}^{\prime}\right\rangle=\lambda_{n}\left|\psi_{n}^{\prime}\right\rangle$, com $\left|\psi_{n}^{\prime}\right\rangle=U\left|\psi_{n}\right\rangle$. Assim, $U$ é um isomorfismo isométrico (preserva o produto escalar) entre os espaços de Hilbert $\mathcal{R}$ e $\mathcal{R}^{\prime}$, associando estados $\left|\psi^{\prime}\right\rangle=U|\psi\rangle$ tais que $\left|\psi^{\prime}\right\rangle$ e $|\psi\rangle$ representam o mesmo estado quântico, descritos em coordenadas diferentes do espaço de fase.

As equações (1.5) e (1.6) têm uma "versão quântica". Consideremos para um índice fixo $\mu$ a função $F(x, p)=\varphi^{\mu}(x)$. Temos $F^{\prime}\left(x^{\prime}, p^{\prime}\right)=x^{\prime \mu}$. Por (1.1), temos $Q^{\prime}\left(F^{\prime}\right)=\hat{x}^{\prime \mu}$. Usando a definição de quantização covariante (1.7), temos portanto a primeira das duas equações abaixo:

$$
\begin{aligned}
\hat{x}^{\prime \mu} & =U Q\left(\varphi^{\mu}(x)\right) U^{\dagger} \\
\hat{p}_{\mu}^{\prime} & =U Q\left(p_{\alpha} \frac{\partial x^{\alpha}}{\partial x^{\prime \mu}}(x)\right) U^{\dagger} .
\end{aligned}
$$

A segunda decorre da definição da função $G(x, p)=p_{\alpha} \frac{\partial x^{\alpha}}{\partial x^{\prime \mu}}(x)$, que implica $G^{\prime}\left(x^{\prime}, p^{\prime}\right)=$ $p_{\mu}^{\prime}$. Estas equações determinam unicamente $U$, a menos de uma fase global. Para verificar esta afirmação, suponhamos que exista $V: \mathcal{R} \rightarrow \mathcal{R}^{\prime}$ inversível que verifique, no lugar de $U$, as equações (1.8) e (1.9). Temos portanto $V^{-1}=V^{\dagger}$. Multiplicando $\hat{x}^{\prime \mu}=V Q\left(\varphi^{\mu}(x)\right) V^{\dagger}$ por $U V^{\dagger}$ à esquerda, temos $U V^{\dagger} \hat{x}^{\prime \mu}=U Q\left(\varphi^{\mu}(x)\right) V^{\dagger}$. Multiplicando (1.8) por $U V^{\dagger}$ à direita, temos $\hat{x}^{\prime \mu} U V^{\dagger}=U Q\left(\varphi^{\mu}(x)\right) V^{\dagger}$, e portanto $\left[U V^{\dagger}, \hat{x}^{\prime \mu}\right]=0$. Analogamente, temos $\left[U V^{\dagger}, \hat{p}_{\mu}^{\prime}\right]=0$. Mas o espaço de Hilbert $\mathcal{R}^{\prime}$ deve ser irredutível em relação aos operadores $\hat{x}^{\prime \mu}$ e $\hat{p}_{\mu}^{\prime}$, portanto $U V^{\dagger}=\lambda \hat{I}$, ou $U=\lambda V$. Ao mesmo tempo, $V U^{\dagger}=\left(U V^{\dagger}\right)^{\dagger}=\lambda^{*} \hat{I}$. Como $U V^{\dagger} V U^{\dagger}=1$, temos $|\lambda|=1$. 


\section{Capítulo 2}

\section{Ordenamento de operadores no espaço plano}

A idéia mais elementar de como construir uma quantização $Q$ que obedeça às condições enumeradas na seção 1.1 é associar a cada função $F(x, p)$ um operador $F(\hat{x}, \hat{p})$. Naturalmente, dada a não comutatividade de $\hat{x}^{\mu}$ e $\hat{p}_{\mu}$, a definição de $F(\hat{x}, \hat{p})$ sofre de ambiguidades, chamadas de ambiguidades de ordenamento, quando $F(x, p)$ contém produtos $x^{\mu} p_{\nu}$, e portanto a cada função $F$ podem, em princípio, corresponder muitos operadores $\hat{F}$. Assim, para um monômio $F(x, p)=x^{\mu_{1}} x^{\mu_{2}} \cdots x^{\mu_{k}} p_{\nu_{1}} p_{\nu_{2}} \cdots p_{\nu_{l}}$, um ordenamento para $F(\hat{x}, \hat{p})$ seria a escolha de uma combinação linear

$$
\begin{aligned}
\hat{F}=\lambda_{1} \hat{x}^{\mu_{1}} \hat{x}^{\mu_{2}} \cdots \hat{x}^{\mu_{k}} \hat{p}_{\nu_{1}} \hat{p}_{\nu_{2}} \cdots \hat{p}_{\nu_{l}}+ & \lambda_{2} \hat{x}^{\mu_{1}} \hat{x}^{\mu_{2}} \cdots \hat{p}_{\nu_{1}} \hat{x}^{\mu_{k}} \hat{p}_{\nu_{2}} \cdots \hat{p}_{\nu_{l}} \\
& +\ldots+\lambda_{(k+l) !} \hat{p}_{\nu_{1}} \hat{p}_{\nu_{2}} \cdots \hat{p}_{\nu_{l}} \hat{x}^{\mu_{1}} \hat{x}^{\mu_{2}} \cdots \hat{x}^{\mu_{k}}
\end{aligned}
$$

onde aparecem na soma a permutação de todos os produtos de operadores

$$
\hat{x}^{\mu_{1}} \hat{x}^{\mu_{2}} \cdots \hat{x}^{\mu_{k}} \hat{p}_{\nu_{1}} \hat{p}_{\nu_{2}} \cdots \hat{p}_{\nu_{l}}
$$

com coeficientes complexos $\lambda$.

Vamos considerar inicialmente o caso mais simples, em que o espaço de fase do sistema clássico é o $\mathbb{R}^{2 D}$ inteiro, isto é, $x \in \mathbb{R}^{D}$ e $p \in \mathbb{R}^{D}$ sem restrições. Mostraremos como certas restrições formais à idéia de quantização juntamente com a imposição de covariância em relação a um grupo de transformações euclidianas ou pseudoeuclidianas podem sistematizar esta idéia, exibindo a forma de todas as quantizações que obedecem a estas restrições. 


\subsection{Algumas propriedades razoáveis para quanti- zações}

No espaço de fase $(x, p) \in \mathbb{R}^{2 D}$, qualquer correspondência $Q$ entre funções complexas $F(x, p)$ e operadores $\hat{F}=Q(F)$ é determinada pela sua ação sobre o "conjunto completo" (no sentido da transformação de Fourier) de funções

$$
e^{i(\eta x+p \xi)}, \eta x \equiv \eta_{\mu} x^{\mu}, p \xi \equiv p_{\mu} \xi^{\mu}
$$

De fato, representando $F(x, p)$ como combinação linear destas funções,

$$
\begin{aligned}
F(x, p) & =\int \tilde{F}(\eta, \xi) e^{i(\eta x+p \xi)} d \eta d \xi \\
\tilde{F}(\eta, \xi) & =\frac{1}{(2 \pi)^{2 D}} \int F(x, p) e^{-i(\eta x+p \xi)} d x d p
\end{aligned}
$$

onde $d \xi=d \xi^{1} d \xi^{2} \cdots d \xi^{D}$ etc, e as integrais são tomadas todas de $-\infty$ a $+\infty$, teremos, de acordo com o princípio de que $Q$ deve ser linear,

$$
Q(F)=\int \tilde{F}(\eta, \xi) Q\left(e^{i(\eta x+p \xi)}\right) d \eta d \xi
$$

Para definir $Q$, começamos com o espaço de Hilbert $\mathcal{R}$ e os $2 D$ operadores $\hat{x}^{\mu}$, $\hat{p}_{\mu}$ que obedecem a (1.2), em relação aos quais $\mathcal{R}$ é irredutível. Os operadores

$$
e^{i(\eta \hat{x}+\hat{p} \xi)}, \eta \hat{x} \equiv \eta_{\mu} \hat{x}^{\mu}, \hat{p} \xi \equiv \hat{p}_{\mu} \xi^{\mu}
$$

em $\mathcal{R}$, dependentes de $2 D$ parâmetros $\eta_{\mu}, \xi^{\mu}$, são completos no conjunto dos operadores do espaço de Hilbert $\mathcal{R}$, da mesma forma que as funções (2.2) são completas no conjunto das funções do espaço de fase (ver o apêndice A). Podemos então definir de maneira geral

$$
Q\left(e^{i(\eta x+p \xi)}\right)=\int \Omega(\eta, \xi, \alpha, \beta) e^{i(\alpha \hat{x}+\hat{p} \beta)} d \alpha d \beta
$$

e, portanto, por (2.5),

$$
Q(F)=\int \tilde{F}(\eta, \xi) \Omega(\eta, \xi, \alpha, \beta) e^{i(\alpha \hat{x}+\hat{p} \beta)} d \alpha d \beta d \eta d \xi
$$

Vamos impor a $Q$ a condição de que os comutadores com $\hat{x}^{\mu}$ e $\hat{p}_{\mu}$ ajam como deri- 
vadas, isto é,

$$
\left[\hat{x}^{\mu}, Q(F)\right]=i \hbar Q\left(\frac{\partial F}{\partial p_{\mu}}\right),\left[\hat{p}_{\mu}, Q(F)\right]=i \hbar Q\left(-\frac{\partial F}{\partial x^{\mu}}\right) .
$$

Estas condições são naturais se pensamos que as equações de Heisenberg para o hamiltoniano $Q(H)$, quantização de de uma hamiltoniana clássica $H$, devam ser formalmente idênticas às equações clássicas de Hamilton:

$$
\dot{\hat{x}}=Q\left(\frac{\partial H}{\partial p_{\mu}}\right), \dot{\hat{p}}=Q\left(-\frac{\partial H}{\partial x^{\mu}}\right) .
$$

Usando o fato de que a transformação de Fourier (2.4) obedece à relação $\frac{\widetilde{\partial F}}{\partial p_{\mu}}(\eta, \xi)=$ $i \xi^{\mu} \tilde{F}(\eta, \xi)$ podemos, usando a expressão (2.6), escrever a primeira das condições (2.7) acima como

$$
\left[\hat{x}^{\mu}, Q(F)\right]=-\hbar \int \xi^{\mu} \tilde{F}(\eta, \xi) \Omega(\eta, \xi, \alpha, \beta) e^{i(\alpha \hat{x}+\hat{p} \beta)} d \alpha d \beta d \eta d \xi
$$

Aplicando as relações de comutação (A.3) sobre (2.6), temos

$$
\left[\hat{x}^{\mu}, Q(F)\right]=-\hbar \int \beta^{\mu} \tilde{F}(\eta, \xi) \Omega(\eta, \xi, \alpha, \beta) e^{i(\alpha \hat{x}+\hat{p} \beta)} d \alpha d \beta d \eta d \xi
$$

Como a função $F$ é geral, devemos ter $\left(\beta^{\mu}-\xi^{\mu}\right) \Omega(\eta, \xi, \alpha, \beta)=0$. Analogamente, pela segunda condição $(2.7),\left(\alpha_{\mu}-\eta_{\mu}\right) \Omega(\eta, \xi, \alpha, \beta)=0$. Estas duas equações somente são possíveis se existe uma função de dois vetores $\Omega(\eta, \xi)$ tal que

$$
\Omega(\eta, \xi, \alpha, \beta)=\Omega(\eta, \xi) \delta(\beta-\xi) \delta(\alpha-\eta)
$$

de modo que (2.6) se expressa como

$$
Q(F)=\int \tilde{F}(\eta, \xi) \Omega(\eta, \xi) e^{i(\eta \hat{x}+\hat{p} \xi)} d \eta d \xi
$$

Assim, quantizações que obedecem às condições (2.7) são obtidas com a correspondência

$$
Q\left(e^{i(\eta x+p \xi)}\right)=Q^{\Omega}\left(e^{i(\eta x+p \xi)}\right)=\Omega(\eta, \xi) e^{i(\eta \hat{x}+\hat{p} \xi)}
$$

onde $\Omega(\eta, \xi)$ é uma função que define $Q^{\Omega}$.

Usualmente, a função $\Omega(\eta, \xi)$ está submetida à condição

$$
\Omega(\eta, 0)=\Omega(0, \xi)=1,
$$


que assegura as chamadas condições de von Neumann

$$
F=F(x) \text { e } G=G(p) \Rightarrow Q^{\Omega}(F)=F(\hat{x}), Q^{\Omega}(G)=G(\hat{p})
$$

em particular as condições de consistência

$$
Q^{\Omega}(1)=\hat{I}, Q^{\Omega}\left(x^{\mu}\right)=\hat{x}^{\mu}, Q^{\Omega}\left(p_{\mu}\right)=\hat{p}_{\mu}
$$

Em (2.11), definimos ${ }^{1}$

$$
\begin{aligned}
& F(\hat{x})=\int \tilde{F}(\eta) e^{i \eta \hat{x}} d \eta, \text { onde } \tilde{F}(\eta)=\frac{1}{(2 \pi)^{D}} \int F(x) e^{-i \eta x} d x \\
& G(\hat{p})=\int \tilde{F}(\xi) e^{i \xi \hat{p}} d \eta, \text { onde } \tilde{G}(\xi)=\frac{1}{(2 \pi)^{D}} \int G(p) e^{-i \xi p} d x
\end{aligned}
$$

As condições de von Neumann (2.11), no entanto, são de certa forma incompletas, já que se referem apenas a funções exclusivamente da posição ou do momento. Se, para um certo índice fixo $\mu, H(x, p)$ não depende de $p_{\mu}$ nem de $x^{\mu}, F\left(x^{\mu}\right)$ é uma função exclusivamente de $x^{\mu}$ e $G\left(p_{\mu}\right)$ é uma função exclusivamente de $p_{\mu}$, é natural exigir que $Q^{\Omega}$ obedeça à condição generalizada

$$
\begin{aligned}
\frac{\partial H}{\partial x^{\mu}}=\frac{\partial H}{\partial p_{\mu}}= & \Rightarrow \\
& \left\{\begin{array}{l}
Q^{\Omega}\left(F\left(x^{\mu}\right) H(x, p)\right)=F\left(\hat{x}^{\mu}\right) Q^{\Omega}(H)=Q^{\Omega}(H) F\left(\hat{x}^{\mu}\right) \\
Q^{\Omega}\left(G\left(p_{\mu}\right) H(x, p)\right)=G\left(\hat{p}_{\mu}\right) Q^{\Omega}(H)=Q^{\Omega}(H) G\left(\hat{p}_{\mu}\right)
\end{array}\right.
\end{aligned}
$$

Deve-se notar que estas condições, juntamente com $Q^{\Omega}(1)=\hat{I}$, implicam (2.11), bastando para isto usar $H=1$.

A condição (2.10), no entanto, não é suficiente para garantir esta condição generalizada. Para tanto, descobrimos as seguintes condições mais estritas:

$$
\frac{\partial}{\partial \eta_{\mu}}\left(\left.\Omega(\eta, \xi)\right|_{\xi^{\mu}=0}\right)=\frac{\partial}{\partial \xi^{\mu}}\left(\left.\Omega(\eta, \xi)\right|_{\eta_{\mu}=0}\right)=0, \Omega(0,0)=1
$$

(sem soma sobre $\mu$ )

Com estas condições, podemos calcular a quantização da função $A(x, p)=F\left(x^{1}\right) H(x, p)$, $\operatorname{com} \frac{\partial H}{\partial x^{1}}=\frac{\partial H}{\partial p_{1}}=0$. Sua transformação de Fourier $(2.4)$ é $\tilde{A}(\eta, \xi)=\bar{F}\left(\eta_{1}\right) \delta\left(\xi^{1}\right) \bar{H}(\eta, \xi)$, onde $\bar{F}$ é a transformação de Fourier de $F$ em relação à variável $x^{1}$ e $\bar{H}$ é a transfor-

\footnotetext{
${ }^{1}$ Estas definições coincidem com a substituição direta $x \rightarrow \hat{x}$ e $p \rightarrow \hat{p}$ em $F(x)$ e $G(p)$ quando $F$ e $G$ são polinomiais.
} 
mação de Fourier de $H$ em relação às variáveis $x^{2} x^{3} \ldots x^{D} p_{2} p_{3} \ldots p_{D}$. Por definição, $\frac{\partial \bar{H}}{\partial \xi^{1}}=\frac{\partial \bar{H}}{\partial \eta_{1}}=0$. Valem então as representações

$$
\begin{gathered}
F\left(x^{1}\right)=\int \bar{F}\left(\eta_{1}\right) e^{\eta_{1} x^{1}} d \eta_{1} \\
H(x, p)=\int \bar{H}(\eta, \xi) \delta\left(\eta_{1}\right) \delta\left(\xi^{1}\right) e^{i(\eta x+p \xi)} d \eta d \xi
\end{gathered}
$$

No cálculo de $Q^{\Omega}(A)(2.8)$, a integração em $\xi^{1}$ é imediata e

$$
\begin{aligned}
Q^{\Omega}(A)= & \int \bar{F}\left(\eta_{1}\right) \bar{H}(\eta, \xi)\left(\Omega(\eta, \xi) e^{i(\eta \hat{x}+\hat{p} \xi)}\right)_{\xi^{1}=0} d \eta d \xi^{2} \cdots d \xi^{D} \\
= & \int \bar{F}\left(\eta_{1}\right) e^{i \eta_{1} \hat{x}^{1}} d \eta_{1} \times \\
& \times\left.\left.\int \bar{H}(\eta, \xi) \Omega(\eta, \xi)\right|_{\xi^{1}=0} e^{i(\eta \hat{x}+\hat{p} \xi)}\right|_{\xi^{1}=\eta_{1}=0} d \eta_{2} \cdots d \eta_{D} d \xi^{2} \cdots d \xi^{D}
\end{aligned}
$$

(Note-se que $\left.e^{i(\eta \hat{x}+\hat{p} \xi)}\right|_{\xi^{1}=0}$ não contém o operador $\hat{p}_{1}$ e, portanto, a separação da parte $e^{i \eta_{1} \hat{x}^{1}}$ da exponencial é possível). A condição (2.16) significa que $\left.\Omega(\eta, \xi)\right|_{\xi^{1}=0}=$ $\left.\Omega(\eta, \xi)\right|_{\xi^{1}=\eta_{1}=0}, \log \mathrm{O}$

$$
\begin{aligned}
Q^{\Omega}(A) & =F\left(\hat{x}^{1}\right) \int \delta\left(\eta_{1}\right) \delta\left(\xi^{1}\right) \bar{H}(\eta, \xi) \Omega(\eta, \xi) e^{i(\eta \hat{x}+\hat{p} \xi)} d \eta d \xi \\
& =F\left(\hat{x}^{1}\right) Q^{\Omega}(H)=Q^{\Omega}(H) F\left(\hat{x}^{1}\right)
\end{aligned}
$$

Analogamente, podemos demonstrar o restante das propriedades (2.15) como conseqüência de (2.16).

Para demonstrar que (2.16) implica em $Q^{\Omega}(1)=\hat{I}$, note-se que por $(2.4) \tilde{1}(\eta, \xi)=$ $\delta(\xi) \delta(\eta)$. Usando $\Omega(0,0)=1,(2.8)$ dá o resultado desejado.

\subsection{Covariância em espaços euclidianos ou pseudo- euclidianos}

Vamos considerar agora que o espaço de configurações $x \in \mathbb{R}^{D}$ seja dotado de uma métrica constante

$$
g_{\mu \nu}=\operatorname{diag}(\underbrace{1,1, \ldots, 1}_{r}, \underbrace{-1,-1, \ldots,-1}_{s})
$$

euclidiana (se $s=0$ ) ou pseudo-euclidiana. Neste espaço são naturais as transformações do grupo ortogonal (se $s=0$ ) ou pseudo-ortogonal $O(r, s)$ cujos elementos 
$M=M^{\mu}{ }_{\nu}$ obedecem a $M^{\alpha}{ }_{\mu} g_{\alpha \beta} M^{\beta}{ }_{\nu}=g_{\mu \nu}$. Este pode ser por exemplo o grupo das rotações e reflexões de $\mathbb{R}^{3}$ se $r=3, s=0$ ou o grupo das transformações de Lorentz se $r=1, s=3$. Neste espaço, o invariante básico é o produto escalar $x \cdot y=g_{\mu \nu} x^{\mu} y^{\nu}=x_{\mu} y^{\mu}$.

No espaço de fase $(x, p) \in \mathbb{R}^{2 D}$, as transformações deste grupo se apresentam como um subgrupo linear das transformações (1.5) e (1.6). A mudança de coordenadas do sistema $x$ para o sistema $x^{\prime}$ são

$$
x^{\prime \alpha}=M_{\mu}^{\alpha} x^{\mu}, p_{\alpha}^{\prime}=p_{\mu}\left(M^{-1}\right)^{\mu}{ }_{\alpha} \text { ou } x^{\prime}=M x, p^{\prime}=p M^{-1} .
$$

Vamos considerar, dentro do conjunto das quantizações $(2.8,2.16)$, quantizações covariantes em relação a este subgrupo. Seja, portanto, $Q^{\Omega}$ uma quantização no sistema de coordenadas $x$, onde $\Omega(\eta, \xi)$ é alguma função que obedece a (2.16). Quaisquer referenciais ortonormais ou pseudo-ortonormais $x^{\mu}$ devem ser tratados como equivalentes. Portanto, no sistema de coordenadas $x^{\prime}$ o procedimento de quantização deve ser $Q^{\prime \Omega}$, usando a mesma função $\Omega(\eta, \xi)$. Assim, a covariâcia do procedimento de quantização (1.7) se expressa como

$$
Q^{\prime \Omega}\left(F^{\prime}\right)=U Q^{\Omega}(F) U^{\dagger}
$$

As equações $(1.8,1.9)$ para $U: \mathcal{R} \rightarrow \mathcal{R}^{\prime}$ são

$$
\hat{x}^{\prime \alpha}=M_{\mu}^{\alpha} U \hat{x}^{\mu} U^{\dagger}, \hat{p}_{\alpha}^{\prime}=U \hat{p}_{\mu} U^{\dagger}\left(M^{-1}\right)^{\mu}{ }_{\alpha}
$$

portanto independentes de $\Omega$.

Consideremos a função $E(x, p)=e^{i(\eta x+p \xi)}$ dependente de $2 D$ parâmetros $\eta_{\mu}, \xi^{\mu}$; no referencial $\left(x^{\prime}, p^{\prime}\right)$, esta função se expressa como $E^{\prime}\left(x^{\prime}, p^{\prime}\right)=e^{i\left(\eta^{\prime} x^{\prime}+p^{\prime} \xi^{\prime}\right)}$, dependente de parâmetros $\eta_{\mu}^{\prime}$, $\xi^{\prime \mu}$, com $\eta_{\alpha}^{\prime}=\eta_{\mu}\left(M^{-1}\right)^{\mu}{ }_{\alpha}$ e $\xi^{\prime \alpha}=M_{\mu}^{\alpha} \xi^{\mu}$. De acordo com (2.9),

$$
\begin{aligned}
Q^{\prime \Omega}\left(E^{\prime}\right) & =\Omega\left(\eta^{\prime}, \xi^{\prime}\right) e^{i\left(\eta^{\prime} \hat{x}^{\prime}+\hat{p}^{\prime} \xi^{\prime}\right)} \\
& =\Omega\left(\eta^{\prime}, \xi^{\prime}\right) U e^{i\left(\eta^{\prime} M \hat{x}+\hat{p} M^{-1} \xi^{\prime}\right)} U^{\dagger} \\
& =U\left(\Omega\left(\eta M^{-1}, M \xi\right) e^{i(\eta \hat{x}+\hat{p} \xi)}\right) U^{\dagger}
\end{aligned}
$$

Comparando com $Q^{\Omega}(E)=\Omega(\eta, \xi) e^{i(\eta \hat{x}+\hat{p} \xi)}$, vemos que $Q^{\prime \Omega}\left(E^{\prime}\right)=U Q^{\Omega}(E) U^{\dagger}$ só pode ser satisfeito se $\Omega\left(\eta M^{-1}, M \xi\right)=\Omega(\eta, \xi)$ (pois $e^{i(\eta \hat{x}+\hat{p} \xi)}$ é um operador inversível). Como $\xi, \eta$ e $M \in O(r, s)$ são arbitrários, $\Omega$ deve ser uma função dos 
invariantes $I_{1}(\xi)=g_{\mu \nu} \xi^{\mu} \xi^{\nu}, I_{2}(\eta)=g^{\mu \nu} \eta_{\mu} \eta_{\nu}$ e $I_{3}(\eta, \xi)=\eta_{\mu} \xi^{\mu}$ :

$$
\Omega(\eta, \xi)=\Omega\left(I_{1}(\xi), I_{2}(\eta), I_{3}(\eta, \xi)\right)
$$

Aplicando a condição (2.16), vemos que

$$
\begin{aligned}
0 & =\frac{\partial}{\partial \xi^{\mu}}\left(\left.\Omega(\eta, \xi)\right|_{\eta_{\mu}=0}\right) \\
& =\frac{\partial}{\partial \xi^{\mu}} \Omega\left(I_{1}(\xi),\left.I_{2}(\eta)\right|_{\eta_{\mu}=0},\left.I_{3}(\eta, \xi)\right|_{\eta_{\mu}=0}\right)=2 g_{\mu \nu} \xi^{\nu} \frac{\partial \Omega}{\partial I_{1}}
\end{aligned}
$$

onde a última igualdade decorre de que $\frac{\partial}{\partial \xi^{\mu}}\left(\left.I_{3}(\eta, \xi)\right|_{\eta_{\mu}=0}\right)=0$. Vemos assim que $\frac{\partial \Omega}{\partial I_{1}}=0$. Usando a outra parte da condição, $\frac{\partial}{\partial \eta_{\mu}}\left(\left.\Omega(\eta, \xi)\right|_{\xi^{\mu}=0}\right)=0$, concluímos analogamente que $\frac{\partial \Omega}{\partial I_{2}}=0$. Assim, para que $Q^{\Omega}$ seja covariante em relação a $O(r, s)$, $\Omega$ deve depender apenas do produto escalar $I_{3}(\eta, \xi)=\eta_{\mu} \xi^{\mu}$ :

$$
\Omega(\eta, \xi)=\Omega\left(\hbar \eta_{\mu} \xi^{\mu}\right)=\Omega(\hbar \eta \xi)
$$

A conveniência introdução do fator $\hbar$ na definição da função de uma variável $\Omega$ ficará clara a seguir. Chegamos assim à expressão de uma quantização $Q^{\Omega}$ covariante em relação a $O(r, s)$ :

$$
Q^{\Omega}(F)=\int \tilde{F}(\eta, \xi) \Omega(\hbar \eta \xi) e^{i(\eta \hat{x}+\hat{p} \xi)} d \eta d \xi
$$

onde $\Omega(k)$ é uma função analítica de uma única variável. Com a forma $\Omega=\Omega(\hbar \eta \xi)$, (2.16) estará completamente satisfeita com

$$
\Omega(0)=1
$$

Podemos definir uma quantização através da função

$$
\Omega_{\theta}(k)=e^{i k \theta}, \text { ou } \Omega_{\theta}(\hbar \eta \xi)=e^{i \hbar \theta \eta \xi}
$$

que claramente obedece a (2.22). A quantização associada é definida por

$$
Q^{\theta}(F)=Q^{\Omega_{\theta}}(F)=\int \tilde{F}(\eta, \xi) e^{i \hbar \theta \eta \xi} e^{i(\eta \hat{x}+\hat{p} \xi)} d \eta d \xi
$$

onde introduzimos a notação abreviada $Q^{\theta}(F)=Q^{\Omega_{\theta}}(F)$ (não há perigo de confusão das notações $Q^{\theta}$ e $Q^{\Omega}$ se se sabe que $\theta$ é um número real e $\Omega$ é uma função). Se 
representarmos uma função arbitrária $\Omega(k)$ por sua transformação de Fourier $\omega(\theta)$

$$
\Omega(k)=\int_{-\infty}^{+\infty} \omega(\theta) e^{i k \theta} d \theta
$$

podemos expressar (2.21) como

$$
Q^{\Omega}(F) \equiv Q_{\omega}(F)=\int_{-\infty}^{+\infty} Q^{\theta}(F) \omega(\theta) d \theta .
$$

Introduzimos também a nova notação $Q_{\omega}$ para quando a quantização estiver expressa em termos da transformação de Fourier $\omega$ de $\Omega$. Da condição (2.22), a definição (2.25) nos dá

$$
\int_{-\infty}^{+\infty} \omega(\theta) d \theta=1
$$

Assim, $\omega$ deve ser uma medida complexa na reta de peso total 1 . Concluímos que (2.26) é a expressão mais geral de uma quantização que obedece a (2.7) e (2.15) e é covariante segundo o grupo $O(r, s)$.

A transformação de Fourier (2.4) da função complexa $F(x, p)$ tem a propriedade $(\tilde{F}(\eta, \xi))^{*}=\widetilde{F}^{*}(-\eta,-\xi)$. Usando a hermiticidade de $\hat{x}^{\mu}$ e $\hat{p}_{\mu}$,

$$
\begin{aligned}
\left(Q^{\theta}(F)\right)^{\dagger} & =\int \widetilde{F^{*}}(-\eta,-\xi) e^{-i \hbar \theta \eta \xi} e^{-i(\eta \hat{x}+\hat{p} \xi)} d \eta d \xi \\
& =\int \widetilde{F^{*}}(\eta, \xi) e^{-i \hbar \theta \eta \xi} e^{i(\eta \hat{x}+\hat{p} \xi)} d \eta d \xi \\
& =Q^{-\theta}\left(F^{*}\right)
\end{aligned}
$$

onde foi efetuada uma mudança de variáveis $(\eta, \xi) \rightarrow(-\eta,-\xi)$ na integral. Vemos assim que a quantização $Q^{\theta}$ é auto-adjunta se e somente se $\theta=0$. Usando esta equação e a definição (2.26), vemos que

$$
\begin{aligned}
\left(Q_{\omega}(F)\right)^{\dagger} & =\int_{-\infty}^{+\infty} Q^{-\theta}\left(F^{*}\right) \omega^{*}(\theta) d \theta \\
& =\int_{-\infty}^{+\infty} Q^{\theta}\left(F^{*}\right) \omega^{*}(-\theta) d \theta=Q_{\omega^{\prime}}\left(F^{*}\right), \\
\omega^{\prime}(\theta) & =\omega^{*}(-\theta) .
\end{aligned}
$$

Em particular, se

$$
\omega^{*}(-\theta)=\omega(\theta) \Leftrightarrow \Omega^{*}(k)=\Omega(k),
$$

então $Q_{\omega}$ é auto-adjunta: $\left(Q_{\omega}(F)\right)^{\dagger}=Q_{\omega}\left(F^{*}\right)$. 
Gostaríamos de quantizar polinômios homogêneos em $p$

$$
F(x, p)=T^{\mu_{1} \cdots \mu_{n}}(x) p_{\mu_{1}} \cdots p_{\mu_{n}}
$$

onde o coeficientes $T^{\mu_{1} \cdots \mu_{n}}(x)$ são simétricos por permutações de qualquer índice $\mu_{1} \cdots \mu_{n}$. Para tanto, vamos apresentar $Q^{\theta}(F)$ de uma maneira conveniente, usando a fórmula (A.1) do apêndice A com $\alpha=\theta+\frac{1}{2}$ :

$$
e^{i(\eta \hat{x}+\hat{p} \xi)}=e^{-i \hbar \theta \eta \xi} e^{i\left(\frac{1}{2}+\theta\right) \hat{p} \xi} e^{i \eta \hat{x}} e^{i\left(\frac{1}{2}-\theta\right) \hat{p} \xi} .
$$

Pela equação (2.24), temos

$$
Q^{\theta}(F)=\int \tilde{F}(\eta, \xi) e^{i\left(\frac{1}{2}+\theta\right) \hat{p} \xi} e^{i \eta \hat{x}} e^{i\left(\frac{1}{2}-\theta\right) \hat{p} \xi} d \eta d \xi
$$

A transformação de Fourier (2.4) de (2.30) é

$$
\tilde{F}(\xi, \eta)=i^{n} \tilde{T}^{\mu_{1} \cdots \mu_{n}}(\eta) \frac{\partial^{n} \delta(\xi)}{\partial \xi^{\mu_{1}} \cdots \partial \xi^{\mu_{n}}}
$$

Substituindo $\tilde{F}$ em (2.31), temos

$$
\begin{aligned}
Q^{\theta}(F) & =i^{n} \int \frac{\partial^{n} \delta(\xi)}{\partial \xi^{\mu_{1}} \cdots \partial \xi^{\mu_{n}}} e^{i\left(\frac{1}{2}+\theta\right) \hat{p} \xi}\left(\int \tilde{T}^{\mu_{1} \cdots \mu_{n}}(\eta) e^{i \eta \hat{x}} d \eta\right) e^{i\left(\frac{1}{2}-\theta\right) \hat{p} \xi} d \xi \\
& =\left.(-i)^{n} \frac{\partial}{\partial \xi^{\mu_{1}}} \cdots \frac{\partial}{\partial \xi^{\mu_{n}}} e^{i\left(\frac{1}{2}+\theta\right) \hat{p} \xi} T^{\mu_{1} \cdots \mu_{n}}(\hat{x}) e^{i\left(\frac{1}{2}-\theta\right) \hat{p} \xi}\right|_{\xi=0} .
\end{aligned}
$$

Usando o resultado do apêndice D, podemos escrever

$$
\begin{aligned}
\frac{\partial}{\partial \xi^{\mu_{1}}} \cdots & \frac{\partial}{\partial \xi^{\mu_{n}}} e^{i\left(\frac{1}{2}+\theta\right) \hat{p} \xi} T^{\mu_{1} \cdots \mu_{n}}(\hat{x}) e^{i\left(\frac{1}{2}-\theta\right) \hat{p} \xi}= \\
& \sum_{k=0}^{n}\left(\begin{array}{l}
n \\
k
\end{array}\right)\left(\frac{\partial}{\partial \xi^{\mu_{1}}} \cdots \frac{\partial}{\partial \xi^{\mu_{k}}} e^{i\left(\frac{1}{2}+\theta\right) \hat{p} \xi}\right) T^{\mu_{1} \cdots \mu_{n}}(\hat{x})\left(\frac{\partial}{\partial \xi^{\mu_{k+1}}} \cdots \frac{\partial}{\partial \xi^{\mu_{n}}} e^{i\left(\frac{1}{2}-\theta\right) \hat{p} \xi}\right)
\end{aligned}
$$

Assim, para um polinômio homogêneo (2.30),

$$
\begin{aligned}
Q^{\theta}\left(T^{\mu_{1} \cdots \mu_{n}}(x) p_{\mu_{1}} \cdots p_{\mu_{n}}\right) & =\sum_{k=0}^{n} C_{k}^{n}(\theta) \hat{p}_{\mu_{1}} \cdots \hat{p}_{\mu_{k}} T^{\mu_{1} \cdots \mu_{n}}(\hat{x}) \hat{p}_{\mu_{k+1}} \cdots \hat{p}_{\mu_{n}} \\
C_{k}^{n}(\theta) & =\left(\begin{array}{l}
n \\
k
\end{array}\right)\left(\frac{1}{2}+\theta\right)^{k}\left(\frac{1}{2}-\theta\right)^{n-k}
\end{aligned}
$$


e a sua quantização $Q_{\omega}$ se expressa então como

$$
\begin{aligned}
Q_{\omega}\left(T^{\mu_{1} \cdots \mu_{n}}(x) p_{\mu_{1}} \cdots p_{\mu_{n}}\right) & =\sum_{k=0}^{n} \omega_{k}^{n} \hat{p}_{\mu_{1}} \cdots \hat{p}_{\mu_{k}} T^{\mu_{1} \cdots \mu_{n}}(\hat{x}) \hat{p}_{\mu_{k+1}} \cdots \hat{p}_{\mu_{n}} \\
\omega_{k}^{n} & =\int_{-\infty}^{+\infty} C_{k}^{n}(\theta) \omega(\theta) d \theta .
\end{aligned}
$$

A soma dos coeficientes $C_{k}^{n}(\theta)$ é um binômio de Newton,

$$
\sum_{k=0}^{n} C_{k}^{n}(\theta)=\left(\left(\frac{1}{2}+\theta\right)+\left(\frac{1}{2}-\theta\right)\right)^{n}=1,
$$

o que implica, levando em conta (2.27), que

$$
\sum_{k=0}^{n} \omega_{k}^{n}=\int_{-\infty}^{+\infty} \sum_{k=0}^{n} C_{k}^{n}(\theta) \omega(\theta) d \theta=1
$$

Assim, ficam claras as formas de $Q^{\theta}$ e $Q^{\Omega}=Q_{\omega}$ como ordenamentos de operadores (2.1). As funções $C_{k}^{n}(\theta)$ têm a propriedade $C_{k}^{n}(-\theta)=C_{n-k}^{n}(\theta)$. Assim, se $Q_{\omega}$ é auto-adjunta, temos

$$
\left(\omega_{k}^{n}\right)^{*}=\int_{-\infty}^{+\infty} C_{k}^{n}(\theta) \omega(-\theta) d \theta=\int_{-\infty}^{+\infty} C_{k}^{n}(-\theta) \omega(\theta) d \theta=\omega_{n-k}^{n} .
$$

\subsection{Exemplos de ordenamentos em espaços eucli- dianos ou pseudo-euclidianos.}

Além das condições $(2.7),(2.15)$, de covariância em relação a $O(r, s)$ e hermiticidade, podemos exigir ainda outras propriedades de uma quantização.

Suponhamos que uma função $F(x, p)$ possa ser decomposta em um produto de funções da forma

$$
F(x, p)=G\left(x^{1}, p_{1}, \ldots, x^{k}, p_{k}\right) H\left(x^{k+1}, p_{k+1}, \ldots x^{D}, p_{D}\right)
$$

Já que para qualquer $Q=Q_{\omega}$ os operadores $Q(G)$ e $Q(H)$ comutam entre si, é natural considerar quais quantizações obedecem a

$$
Q(F)=Q(G) Q(H)
$$


As transformações de Fourier (2.4) de $G, H$ e $F$ têm a forma

$$
\begin{aligned}
\tilde{G}(\eta, \xi) & =\bar{G}\left(\eta_{1}, \xi^{1}, \ldots, \eta_{k}, \xi^{k}\right) \delta\left(\eta_{k+1}\right) \delta\left(\xi^{k+1}\right) \ldots \delta\left(\eta_{D}\right) \delta\left(\xi^{D}\right) \\
\tilde{H}(\eta, \xi) & =\bar{H}\left(\eta_{k+1}, \xi^{k+1}, \ldots, \eta_{D}, \xi^{D}\right) \delta\left(\eta_{1}\right) \delta\left(\xi^{1}\right) \ldots \delta\left(\eta_{k}\right) \delta\left(\xi^{k}\right) \\
\tilde{F}(\eta, \xi) & =\bar{G}\left(\eta_{1}, \xi^{1}, \ldots, \eta_{k}, \xi^{k}\right) \bar{H}\left(\eta_{k+1}, \xi^{k+1}, \ldots, \eta_{D}, \xi^{D}\right) .
\end{aligned}
$$

Devido às relações de comutação $\left[\sum_{\mu=1}^{k}\left(\eta_{\mu} \hat{x}^{\mu}+\hat{p}_{\mu} \xi^{\mu}\right), \sum_{\mu=k+1}^{D}\left(\eta_{\mu} \hat{x}^{\mu}+\hat{p}_{\mu} \xi^{\mu}\right)\right]=0$, temos $e^{i(\eta \hat{x}+\hat{p} \xi)}=e^{i \sum_{\mu=1}^{k}\left(\eta_{\mu} \hat{x}^{\mu}+\hat{p}_{\mu} \xi^{\mu}\right)} e^{i \sum_{\mu=k+1}^{D}\left(\eta_{\mu} \hat{x}^{\mu}+\hat{p}_{\mu} \xi^{\mu}\right)}$, e, portanto, a quantização $Q^{\theta}$ dada por (2.24) pode ser escrita como

$$
\begin{aligned}
Q^{\theta}(F)= & \int \bar{G} e^{i \hbar \theta \sum_{\mu=1}^{k} \eta_{\mu} \xi^{\mu}} e^{i \sum_{\mu=1}^{k}\left(\eta_{\mu} \hat{x}^{\mu}+\hat{p}_{\mu} \xi^{\mu}\right)} \prod_{\mu=1}^{k} d \eta_{\mu} d \xi^{\mu} \times \\
& \times \int \bar{H} e^{i \hbar \theta \sum_{\mu=k+1}^{D} \eta_{\mu} \xi^{\mu}} e^{i \sum_{\mu=k+1}^{D}\left(\eta_{\mu} \hat{x}^{\mu}+\hat{p}_{\mu} \xi^{\mu}\right)} \prod_{\mu=k+1}^{D} d \eta_{\mu} d \xi^{\mu} \\
= & \int \tilde{G} e^{i \hbar \theta \eta_{\mu} \xi^{\mu}} e^{i\left(\eta_{\mu} \hat{x}^{\mu}+\hat{p}_{\mu} \xi^{\mu}\right)} d \eta d \xi \int \tilde{H} e^{i \hbar \theta \eta_{\mu} \xi^{\mu}} e^{i\left(\eta_{\mu} \hat{x}^{\mu}+\hat{p}_{\mu} \xi^{\mu}\right)} d \eta d \xi \\
= & Q^{\theta}(G) Q^{\theta}(H),
\end{aligned}
$$

logo a quantização $Q^{\theta}$ obedece à condição (2.38) para qualquer $\theta$. Vamos demonstrar que, entre as quantizações $(2.26)$, somente as $Q^{\theta}(F)$ obedecem a esta condição. De fato, para (2.37),

$$
\begin{aligned}
Q_{\omega}(F) & =\int Q^{\theta}(F) \omega(\theta) d \theta=\int Q^{\theta}(G) Q^{\theta}(H) \omega(\theta) d \theta \\
& =\int Q^{\theta_{1}}(G) Q^{\theta_{2}}(H) \omega\left(\theta_{1}\right) \delta\left(\theta_{2}-\theta_{1}\right) d \theta_{1} d \theta_{2}
\end{aligned}
$$

e, por outro lado,

$$
Q_{\omega}(G) Q_{\omega}(H)=\int Q^{\theta_{1}}(G) Q^{\theta_{2}}(H) \omega\left(\theta_{1}\right) \omega\left(\theta_{2}\right) d \theta_{1} d \theta_{2}
$$

A equação (2.32) mostra que as funções $G$ e $H$ podem ser escolhidas de modo que $Q^{\theta_{1}}(G) Q^{\theta_{2}}(H)$ seja um polinômio (com coeficientes operatoriais) em $\theta_{1}$ e $\theta_{2}$ de grau arbitrário. Assim, a condição (2.38) será satisfeita somente se

$$
\omega\left(\theta_{1}\right) \omega\left(\theta_{2}\right)=\omega\left(\theta_{1}\right) \delta\left(\theta_{2}-\theta_{1}\right)
$$

Levando em conta a propriedade $f\left(\theta_{1}\right) \delta\left(\theta_{2}-\theta_{1}\right)=f\left(\theta_{2}\right) \delta\left(\theta_{2}-\theta_{1}\right)$ da função 


\section{CAPÍTULO 2. ORDENAMENTO DE OPERADORES NO ESPAÇO PLANO 20}

delta, para uma função arbitrária $f(\theta)$, a equação acima implica em

$$
f\left(\theta_{1}\right) \omega\left(\theta_{1}\right) \omega\left(\theta_{2}\right)=f\left(\theta_{2}\right) \omega\left(\theta_{1}\right) \omega\left(\theta_{2}\right) .
$$

Integrando em $\theta_{2}$ e levando em conta (2.27),

$$
f\left(\theta_{1}\right) \omega\left(\theta_{1}\right)=k \omega\left(\theta_{1}\right), k=\int_{-\infty}^{\infty} f(\theta) \omega(\theta) d \theta
$$

Isto só é possível para toda $f$ se $\omega(\theta)=\delta(\theta-\lambda)(k$ será então $f(\lambda))$, o que implica que $Q_{\omega}(F)=Q^{\lambda}(F)$, o que demonstra a proposição.

Entre as $Q^{\theta}(F)$, destacam-se as quantizações $Q^{x p}(F)=Q^{-\frac{1}{2}}(F)$ e $Q^{p x}(F)=$ $Q^{\frac{1}{2}}(F)$, obtidas usando $\theta=-\frac{1}{2}$ e $\theta=\frac{1}{2}$, respectivamente. Pelas fórmulas (2.31) e, particularmente, $(2.32)$, vemos que $Q^{x p}(F)$ se consegue substituindo em $F(x, p)$ todos os operadores $\hat{x}^{\mu}$ à esquerda dos operadores $\hat{p}_{\mu}$ e $Q^{p x}(F)$ se consegue substituindo em $F(x, p)$ todos os operadores $\hat{p}_{\mu}$ à esquerda dos operadores $\hat{x}^{\mu}$. De acordo com a fórmula $(2.28),\left(Q^{x p}(F)\right)^{\dagger}=Q^{p x}(F)$, e, portanto, em geral $Q^{x p}(F)$ e $Q^{p x}(F)$ não são operadores auto-adjuntos.

A fórmula (2.28) mostra que a única quantização $Q^{\theta}(F)$ auto-adjunta é a quantização de Weyl $Q^{\mathrm{W}}(F)=Q^{0}(F)$, obtida usando $\theta=0$. Assim, a quantização de Weyl é a única que obedece às propriedades já discutidas, juntamente com (2.38) e hermiticidade. Para a quantização de Weyl, temos $\omega(\theta)=\delta(\theta)$, por (2.23) $\Omega=\Omega_{0}=1$ e por $(2.32)$

$$
Q^{\mathrm{W}}\left(T^{\mu_{1} \cdots \mu_{n}}(x) p_{\mu_{1}} \cdots p_{\mu_{n}}\right)=\frac{1}{2^{n}} \sum_{k=0}^{n}\left(\begin{array}{l}
n \\
k
\end{array}\right) \hat{p}_{\mu_{1}} \cdots \hat{p}_{\mu_{k}} T^{\mu_{1} \cdots \mu_{n}}(\hat{x}) \hat{p}_{\mu_{k+1}} \cdots \hat{p}_{\mu_{n}}
$$

Uma quantização que não pertence à classe $Q^{\theta}(F)$ é a generalização multidimensional da quantização de Born-Jordan, obtida com a função

$$
\omega(\theta)=\left\{\begin{array}{ll}
1 & \text { se }-1 / 2<\theta<1 / 2 \\
0 & \text { de outra maneira }
\end{array}=\chi_{(-1 / 2,1 / 2)}(\theta),\right.
$$

onde $\chi_{(-1 / 2,1 / 2)}$ é a função característica do intervalo $(-1 / 2,1 / 2)$ :

$$
Q^{\mathrm{BJ}}(F)=\int_{-\frac{1}{2}}^{+\frac{1}{2}} Q^{\theta}(F) d \theta
$$

Esta generalização não foi apresentada previamente e é não trivial, já que a quantização de Born-Jordan não obedece a (2.38). Sua característica distintiva é que, se 
$F=F(x)$ e $G=G(p)$,

$$
[F(\hat{x}), G(\hat{p})]=i \hbar Q^{\mathrm{BJ}}(\{F, G\}) \equiv i \hbar Q^{\mathrm{BJ}}\left(\frac{\partial F}{\partial x^{\mu}} \frac{\partial G}{\partial p_{\mu}}\right) .
$$

Para demonstrar esta proposição, notemos que, devido a (2.4),

$$
\widetilde{\frac{\partial F}{\partial x^{\mu}} \frac{\partial G}{\partial p_{\mu}}}(\eta, \xi)=-\eta_{\mu} \xi^{\mu} \widetilde{F G}(\eta, \xi) .
$$

Assim, usando as definições (2.21) e (2.23),

$$
Q^{\theta}\left(\frac{\partial F}{\partial x^{\mu}} \frac{\partial G}{\partial p_{\mu}}\right)=-\int \widetilde{F G} \eta_{\mu} \xi^{\mu} e^{i \hbar \theta \eta \eta \xi} e^{i(\eta \hat{x}+\hat{p} \xi)} d \eta d \xi=-\frac{1}{i \hbar} \frac{\partial}{\partial \theta} Q^{\theta}(F G)
$$

e, portanto,

$$
\begin{aligned}
Q^{\mathrm{BJ}}\left(\frac{\partial F}{\partial x^{\mu}} \frac{\partial G}{\partial p_{\mu}}\right) & =-\frac{1}{i \hbar} \int_{-\frac{1}{2}}^{+\frac{1}{2}} \frac{\partial}{\partial \theta} Q^{\theta}(F G) d \theta=\frac{1}{i \hbar}\left(Q^{-\frac{1}{2}}(F G)-Q^{\frac{1}{2}}(F G)\right) \\
& =\frac{1}{i \hbar}\left(Q^{x p}(F G)-Q^{p x}(F G)\right)=\frac{1}{i \hbar}(F(\hat{x}) G(\hat{p})-G(\hat{p}) F(\hat{x})) \\
& =\frac{1}{i \hbar}[F(\hat{x}), G(\hat{p})],
\end{aligned}
$$

o que demonstra a proposição. Para a quantização de Born-Jordan, temos por (2.25) $\Omega(\hbar \eta \xi)=\frac{2}{\hbar \eta \xi} \sin \left(\frac{\hbar \eta \xi}{2}\right)$. Por $(2.32)$

$$
\begin{gathered}
\omega_{k}^{n}=\left(\begin{array}{l}
n \\
k
\end{array}\right) \int_{-\frac{1}{2}}^{+\frac{1}{2}}\left(\frac{1}{2}+\theta\right)^{k}\left(\frac{1}{2}-\theta\right)^{n-k} d \theta=\frac{1}{n+1} \\
Q^{\mathrm{BJ}}\left(T^{\mu_{1} \cdots \mu_{n}}(x) p_{\mu_{1}} \cdots p_{\mu_{n}}\right)=\frac{1}{n+1} \sum_{k=0}^{n} \hat{p}_{\mu_{1}} \cdots \hat{p}_{\mu_{k}} T^{\mu_{1} \cdots \mu_{n}}(\hat{x}) \hat{p}_{\mu_{k+1}} \cdots \hat{p}_{\mu_{n}}
\end{gathered}
$$

\subsection{Símbolos de operadores e limite clássico em espaços euclidianos ou pseudo-euclidianos}

Para o ordenamento de Weyl, temos $\Omega(\hbar \eta \xi)=1$, e, portanto,

$$
Q^{\mathrm{W}}(F)=\int \tilde{F}(\eta, \xi) e^{i(\eta \hat{x}+\hat{p} \xi)} d \eta d \xi
$$


Comparando com a forma geral $(2.21)$, vemos que $Q^{\Omega}(F)=Q^{\mathrm{W}}(G)$, onde $\tilde{G}(\eta, \xi)=$ $\Omega(\hbar \eta \xi) \tilde{F}(\eta, \xi)$. Pela definição $(2.3)$,

$$
\Omega(-\hbar \Delta) F(x, p)=\int \Omega(\hbar \eta \xi) \tilde{F}(\eta, \xi) e^{i(\eta x+p \xi)} d \eta d \xi
$$

onde

$$
\Delta \equiv \sum_{\mu=1}^{D} \frac{\partial^{2}}{\partial x^{\mu} \partial p_{\mu}}
$$

Assim,

$$
\Omega(\hbar \eta \xi) \tilde{F}(\eta, \xi)=(\Omega(-\hbar \Delta) F)^{\sim} .
$$

Observemos que se $F(x, p)$ é polinomial em $x$ ou $p$, existe uma potência $n$ tal que $\Delta^{n} F=0$, e portanto para tais funções $\Omega(-\hbar \Delta) F$ sempre existe. Assim,

$$
Q^{\Omega}(F)=Q^{\mathrm{W}}(\Omega(-\hbar \Delta) F)
$$

O operador $\Omega(-\hbar \Delta)$ tem inversa, já que $\tilde{F}(\eta, \xi)=[\Omega(\hbar \eta \xi)]^{-1} \tilde{G}(\eta, \xi)$, e, portanto, $F=[\Omega(-\hbar \Delta)]^{-1} G$.

Quando $\hat{F}=Q^{\Omega}(F)$, diremos que a função $F(x, p)$ é o $\Omega$-símbolo do operador $\hat{F}$ (ver propriedade 4 da seção 1.1). Denotaremos por $F *_{\Omega} G$ o produto de $\Omega$-símbolos. $\mathrm{O}$ produto $*_{\mathrm{w}}$ de símbolos de Weyl é dado por

$$
\begin{aligned}
\left(F *_{\mathrm{W}} G\right)(x, p) & =\exp \left(\frac{i \hbar}{2}\left(\frac{\partial^{2}}{\partial x_{1} \partial p_{2}}-\frac{\partial^{2}}{\partial x_{2} \partial p_{1}}\right)\right) F\left(x_{1}, p_{1}\right) G\left(x_{2}, p_{2}\right) \mid \begin{array}{r}
x_{1}=x_{2}=x \\
p_{1}=p_{2}=p
\end{array} \\
& =F(x, p) G(x, p)+\frac{i \hbar}{2}\{F, G\}(x, p)+O\left(\hbar^{2}\right) .
\end{aligned}
$$

Pela definição do produto de $\Omega$-símbolos, temos

$$
Q^{\Omega}\left(F *_{\Omega} G\right)=Q^{\Omega}(F) Q^{\Omega}(G)
$$

Expressando $Q^{\Omega}$ em termos de $Q^{\mathrm{W}}$ e usando a definição de produto de símbolos de Weyl, a igualdade acima fica

$$
\begin{aligned}
Q^{\mathrm{W}}\left(\Omega(-\hbar \Delta)\left(F *_{\Omega} G\right)\right) & =Q^{\mathrm{W}}(\Omega(-\hbar \Delta) F) Q^{\mathrm{W}}(\Omega(-\hbar \Delta) G) \\
& =Q^{\mathrm{W}}\left([\Omega(-\hbar \Delta) F] *_{\mathrm{W}}[\Omega(-\hbar \Delta) G]\right)
\end{aligned}
$$


Igualando os argumentos de $Q^{\mathrm{W}}$ no primeiro e no último membros desta equação,

$$
F *_{\Omega} G=[\Omega(-\hbar \Delta)]^{-1}\left([\Omega(-\hbar \Delta) F] *_{\mathrm{W}}[\Omega(-\hbar \Delta) G]\right) .
$$

Usando (2.22), temos $\Omega(-\hbar \Delta)=1-a \hbar \Delta+O\left(\hbar^{2}\right)$, onde $a=d \Omega(k) /\left.d k\right|_{k=0}$. Usando a expansão (2.40), e levando em conta que $[\Omega(-\hbar \Delta)]^{-1}=1+a \hbar \Delta+O\left(\hbar^{2}\right)$,

$$
F *_{\Omega} G=F G+\frac{i \hbar}{2}\{F, G\}+\left.\frac{d \Omega(k)}{d k}\right|_{k=0} \hbar \sum_{\mu=1}^{D}\left(\frac{\partial F}{\partial x^{\mu}} \frac{\partial G}{\partial p_{\mu}}+\frac{\partial G}{\partial x^{\mu}} \frac{\partial F}{\partial p_{\mu}}\right)+O\left(\hbar^{2}\right)
$$

e também

$$
F *_{\Omega} G-G *_{\Omega} F=i \hbar\{F, G\}+O\left(\hbar^{2}\right) .
$$

Estas duas equações mostram que o produto $*_{\Omega}$ obedece às condições (1.3) e (1.4), o que prova o limite clássico para a quantização $Q^{\Omega}$.

\subsection{Uma forma alternativa para $Q^{\theta}$ e $Q_{\omega}$}

Nosso objetivo na próxima seção é estender as quantizações $Q_{\omega}(2.26)$ a sistemas de coordenadas $x^{\prime}=\varphi(x)$ gerais. Na expressão (2.26), (2.24) é claramente inadequada quando se usam coordenadas gerais, pois usa a transformação de Fourier $\tilde{F}$ (2.3), (2.4) que não tem uma boa lei de transformação sob transformações gerais de coordenadas. $\tilde{F}$ pode em geral nem existir, já que coordenadas gerais podem não se estender de $-\infty$ a $+\infty$. Assim, vamos expressar (2.24) de uma forma em que não apareça a transformação de Fourier sobre a variável $x$.

Daqui em diante vamos representar a transformação de Fourier $(2.4)$ de $F(x, p)$ em relação às duas variáveis $x$ e $p$ por $\tilde{\tilde{F}}(\eta, \xi)$. A notação $\tilde{F}(x, \xi)$ será usada para a transformação de Fourier parcial em relação à variável $p^{2}$,

$$
\begin{aligned}
\tilde{F}(x, \xi) & =\frac{1}{(2 \pi \hbar)^{D}} \int F(x, p) e^{-\frac{i}{\hbar} p \xi} d p \\
F(x, p) & =\int \tilde{F}(x, \xi) e^{\frac{i}{\hbar} p \xi} d \xi
\end{aligned}
$$

Se em coordenadas $x$ e $x^{\prime}=\varphi(x)$ o tensor métrico tem formas gerais quaisquer

\footnotetext{
${ }^{2}$ Para minimizar as ocorrências de $\hbar$ nas fórmulas a seguir, introduzimos esta constante na exponencial complexa.
} 
$g_{\mu \nu}(x)$ e $g_{\mu \nu}^{\prime}\left(x^{\prime}\right)$ respectivamente $^{3}$, e $F(x, p)=F^{\prime}\left(x^{\prime}, p^{\prime}\right)$ com $p_{\mu}^{\prime}=p_{\nu} \frac{\partial x^{\nu}}{\partial x^{\prime \mu}}$, então

$$
\begin{aligned}
\tilde{F}(x, \xi) & =\frac{1}{(2 \pi \hbar)^{D}} \int F^{\prime}\left(x^{\prime}, p \frac{\partial x}{\partial x^{\prime}}\right) e^{-\frac{i}{\hbar} p \xi} d p \\
& =\frac{1}{(2 \pi \hbar)^{D}} \int F^{\prime}\left(x^{\prime}, p^{\prime}\right) e^{-\frac{i}{\hbar} p^{\prime} \frac{\partial x^{\prime}}{\partial x} \xi}\left|\operatorname{det}\left(\frac{\partial x^{\prime}}{\partial x}\right)\right| d p^{\prime} \\
& =\frac{\sqrt{g(x)}}{\sqrt{g^{\prime}\left(x^{\prime}\right)}} \widetilde{F^{\prime}}\left(x^{\prime}, \xi^{\prime}\right),
\end{aligned}
$$

com $\xi^{\prime \mu}=\frac{\partial x^{\prime \mu}}{\partial x^{\nu}} \xi^{\nu}$, onde foi usada a relação (B.3), com $g=\left|\operatorname{det}\left(g_{\mu \nu}\right)\right|$ e $g^{\prime}=$ $\left|\operatorname{det}\left(g_{\mu \nu}^{\prime}\right)\right|$. Assim, a lei de transformação de $\tilde{F}$ é dada por

$$
\frac{\tilde{F}(x, \xi)}{\sqrt{g(x)}}=\frac{\widetilde{F^{\prime}}\left(x^{\prime}, \xi^{\prime}\right)}{\sqrt{g^{\prime}\left(x^{\prime}\right)}}
$$

Vamos partir da forma (2.31):

$$
Q^{\theta}(F)=\int e^{i\left(\frac{1}{2}+\theta\right) \hat{p} \xi}\left(\int \tilde{\tilde{F}}(\eta, \xi) e^{i \eta \hat{x}} d \eta\right) e^{i\left(\frac{1}{2}-\theta\right) \hat{p} \xi} d \xi
$$

Podemos expressar $\tilde{F}$ em termos de $\tilde{\tilde{F}}$ como

$$
\begin{aligned}
& \int \tilde{\tilde{F}}(\eta, \xi) e^{i \eta x} d \eta=\frac{1}{(2 \pi)^{D}} \int F(x, p) e^{-i p \xi} d p \\
&=\hbar^{D} \frac{1}{(2 \pi \hbar)^{D}} \int F(x, p) e^{-\frac{i}{\hbar} p(\hbar \xi)} d p=\hbar^{D} \tilde{F}(x, \hbar \xi)
\end{aligned}
$$

e, portanto, por (2.13),

$$
\int \tilde{\tilde{F}}(\eta, \xi) e^{i \eta \hat{x}} d \eta=\hbar^{D} \tilde{F}(\hat{x}, \hbar \xi)=\hbar^{D} \int \tilde{F}(x, \hbar \xi)|x\rangle\langle x| d x .
$$

Em um sistema de coordenadas retilíneas $x$, os auto-estados da posição $|x\rangle$ são definidos da maneira usual ${ }^{4}$ :

$$
\hat{x}^{\mu}|x\rangle=x^{\mu}|x\rangle,\langle x \mid y\rangle=\delta(x-y),\langle x| \hat{p}_{\mu}=-i \hbar \frac{\partial}{\partial x^{\mu}}\langle x| .
$$

\footnotetext{
${ }^{3}$ Podem ser coordenadas gerais em um espaço plano ou curvo.

${ }^{4} \mathrm{~A}$ última equação de (2.46) é necessária para fixar uma fase dos vetores $|x\rangle$. A forma mais geral da representação da posição para operadores $\hat{p}_{\mu}$ que verifiquem as relações de comutação (1.2) é $\left\langle x\left|\hat{p}_{\mu}\right| \psi\right\rangle=\left(-i \hbar \frac{\partial}{\partial x^{\mu}}+\frac{\partial \phi(x)}{\partial x^{\mu}}\right)\langle x \mid \psi\rangle$. Redefinindo os vetores da posição por $|x\rangle \rightarrow e^{i \phi(x) / \hbar}|x\rangle$ chegamos à expressão usual $\left\langle x\left|\hat{p}_{\mu}\right| \psi\right\rangle=-i \hbar \frac{\partial}{\partial x^{\mu}}\langle x \mid \psi\rangle$.
} 
Dada a sua completude, implicam na representação

$$
\hat{I}=\int|x\rangle\langle x| d x
$$

A última igualdade de (2.45) pode ser verificada imediatamente aplicando o operador $\hbar^{D} \int \tilde{F}(x, \hbar \xi)|x\rangle\langle x| d x$ sobre o vetor $|y\rangle$, usando a segunda equação de (2.46).

Substituindo (2.45) em (2.44),

$$
\begin{aligned}
Q^{\theta}(F) & =\hbar^{D} \int \tilde{F}(x, \hbar \xi) e^{i\left(\frac{1}{2}+\theta\right) \hat{p} \xi}|x\rangle\langle x| e^{i\left(\frac{1}{2}-\theta\right) \hat{p} \xi} d x d \xi \\
& =\int \tilde{F}(x, \xi) e^{\frac{i}{\hbar}\left(\frac{1}{2}+\theta\right) \hat{p} \xi}|x\rangle\langle x| e^{\frac{i}{\hbar}\left(\frac{1}{2}-\theta\right) \hat{p} \xi} d x d \xi
\end{aligned}
$$

A última equação de (2.46), que mostra a forma explícita do operador de momento na representação da posição, pode ser usada para calcular

$$
\begin{aligned}
& \langle x| e^{\frac{i}{\hbar}\left(\frac{1}{2}-\theta\right) \hat{p} \xi}=e^{\left(\frac{1}{2}-\theta\right) \xi^{\mu} \frac{\partial}{\partial x^{\mu}}}\langle x|=\left\langle x+\left(\frac{1}{2}-\theta\right) \xi\right| \\
& e^{\frac{i}{\hbar}\left(\frac{1}{2}+\theta\right) \hat{p} \xi}|x\rangle=\left(\langle x| e^{-\frac{i}{\hbar}\left(\frac{1}{2}+\theta\right) \hat{p} \xi}\right)^{\dagger}=\left|x-\left(\frac{1}{2}+\theta\right) \xi\right\rangle
\end{aligned}
$$

$\left(e^{a^{\mu} \frac{\partial}{\partial x^{\mu}}} f(x)\right.$ é a expansão de Tailor de $f(x+a)$ em torno $\left.x\right)$ e, portanto,

$$
Q^{\theta}(F)=\int \tilde{F}(x, \xi)\left|x-\left(\frac{1}{2}+\theta\right) \xi\right\rangle\left\langle x+\left(\frac{1}{2}-\theta\right) \xi\right| d x d \xi,
$$

forma em que não aparece a transformação de Fourier de $F$ em relação à variável $x$. A quantização $Q_{\omega}$ se expressa através da equação (2.26) como

$$
Q_{\omega}(F)=\int \tilde{F}(x, \xi) \hat{D}(x, \xi, \omega) d x d \xi
$$

onde definimos o operador em $\mathcal{R}$

$$
\hat{D}(x, \xi, \omega)=\int\left|x-\left(\frac{1}{2}+\theta\right) \xi\right\rangle\left\langle x+\left(\frac{1}{2}-\theta\right) \xi\right| \omega(\theta) d \theta .
$$

Se $F(x, p)$ é polinomial em $p$, podemos expressar $\tilde{F}(x, \xi)(2.41)$ como

$$
\tilde{F}(x, \xi)=F\left(x, i \hbar \frac{\partial}{\partial \xi}\right) \frac{1}{(2 \pi \hbar)^{D}} \int e^{-\frac{i}{\hbar} p \xi} d p=F\left(x, i \hbar \frac{\partial}{\partial \xi}\right) \delta(\xi),
$$


e portanto (2.48) como

$$
Q_{\omega}(F)=\left.\int F\left(x,-i \hbar \frac{\partial}{\partial \xi}\right) \hat{D}(x, \xi, \omega)\right|_{\xi=0} d x .
$$

\subsection{Quantização em coordenadas gerais}

Nesta seção, vamos estender as quantizações $Q_{\omega}$ auto-adjuntas a coordenadas gerais (1.5) $x^{\prime}=\varphi(x)$. No sistema de coordenadas arbitrário $x^{\prime}$, definimos um espaço de Hilbert $\mathcal{R}^{\prime}$ com $2 D$ operadores $\hat{x}^{\prime \mu}, \hat{p}_{\mu}^{\prime}$ obedecendo as condições do ítem 3 da seção 1.1. As equações (1.8) e (1.9) definem a transformação $U: \mathcal{R} \rightarrow \mathcal{R}^{\prime}$ que, em geral dependerão de $\omega$. Definiremos então a quantização $Q_{\omega}^{\prime}$ nas coordenadas $x^{\prime}$ por

$$
Q_{\omega}^{\prime}\left(F^{\prime}\right)=U Q_{\omega}(F) U^{\dagger}
$$

que coincide, por construção, com o ordenamento de operadores (2.26) quando o sistema de coordenadas $x^{\prime}$ é ortonormal. Iremos mostrar que a quantização $Q_{\omega}^{\prime}$ pode ser exibida usando apenas elementos intrínsecos ao sistema de coordenadas $x^{\prime}$, e portanto, de forma independente do sistema de coordenadas euclidianas original $x$.

Precisamos exibir a forma concreta das equações (1.8) e (1.9) para $Q_{\omega}$. Pelas propriedades (2.11), temos $Q_{\omega}\left(\varphi^{\mu}(x)\right)=\varphi^{\mu}(\hat{x})$, e, portanto, a equação (1.8) se torna

$$
\hat{x}^{\prime \mu}=U \varphi^{\mu}(\hat{x}) U^{\dagger},
$$

independente de $\omega$. Usando a notação

$$
\frac{\widehat{\partial x^{\alpha}}}{\partial x^{\prime \mu}} \equiv \frac{\partial x^{\alpha}}{\partial x^{\prime \mu}}(\varphi(\hat{x}))
$$

Pela expressão (2.34),

$$
Q_{\omega}\left(p_{\alpha} \frac{\partial x^{\alpha}}{\partial x^{\prime \mu}}\right)=\omega_{0}^{1} \frac{\widehat{\partial x^{\alpha}}}{\partial x^{\prime \mu}} \hat{p}_{\alpha}+\omega_{1}^{1} \hat{p}_{\alpha} \frac{\widehat{\partial x^{\alpha}}}{\partial x^{\prime \mu}}
$$


Os coeficientes $\omega_{0}^{1}$ e $\omega_{1}^{1}$ são dados por

$$
\begin{aligned}
& \omega_{0}^{1}=\int\left(\frac{1}{2}-\theta\right) \omega(\theta) d \theta=\frac{1}{2}+i \omega^{1} \\
& \omega_{1}^{1}=\int\left(\frac{1}{2}+\theta\right) \omega(\theta) d \theta=\frac{1}{2}-i \omega^{1} \\
& \omega^{1}=i \int \theta \omega(\theta) d \theta=\left.\frac{d \Omega}{d k}\right|_{k=0},
\end{aligned}
$$

onde definimos a quantidade $\omega^{1}$ e a última equação decorre de (2.25). Portanto,

$$
Q_{\omega}\left(p_{\alpha} \frac{\partial x^{\alpha}}{\partial x^{\prime \mu}}\right)=\frac{1}{2}\left(\frac{\widehat{\partial x^{\alpha}}}{\partial x^{\prime \mu}} \hat{p}_{\alpha}+\hat{p}_{\alpha} \frac{\widehat{\partial x^{\alpha}}}{\partial x^{\prime \mu}}\right)-i \omega^{1}\left[\hat{p}_{\alpha}, \frac{\widehat{\partial x^{\alpha}}}{\partial x^{\prime \mu}}\right] .
$$

Pelas condições (2.7),

$$
\left[\hat{p}_{\alpha}, \widehat{\frac{\partial x^{\alpha}}{\partial x^{\prime \mu}}}\right]=\left[\hat{p}_{\alpha}, Q_{\omega}\left(\frac{\partial x^{\alpha}}{\partial x^{\prime \mu}}(\varphi(\hat{x}))\right)\right]=-i \hbar Q_{\omega}\left(\frac{\partial}{\partial x^{\alpha}}\left(\frac{\partial x^{\alpha}}{\partial x^{\prime \mu}}\right)\right) .
$$

Temos $\frac{\partial}{\partial x^{\alpha}}\left(\frac{\partial x^{\alpha}}{\partial x^{\prime \mu}}\right)=\frac{\partial^{2} x^{\alpha}}{\partial x^{\prime \mu} \partial x^{\prime \nu}} \frac{\partial x^{\prime \nu}}{\partial x^{\alpha}}=\Gamma_{\alpha \mu}^{\prime \alpha} \equiv \Gamma_{\mu}^{\prime}$, onde $\Gamma_{\mu \nu}^{\prime \alpha}$ é a conexão de Levi-Civita no sistema de coordenadas $x^{\prime}$ (ver equação (B.9) do apêndice B). Assim, o comutador se expressa como

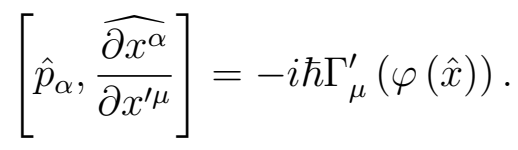

Obtemos assim finalmente

$$
Q_{\omega}\left(p_{\alpha} \frac{\partial x^{\alpha}}{\partial x^{\prime \mu}}\right)=\frac{1}{2}\left(\frac{\widehat{\partial x^{\alpha}}}{\partial x^{\prime \mu}} \hat{p}_{\alpha}+\hat{p}_{\alpha} \frac{\widehat{\partial x^{\alpha}}}{\partial x^{\prime \mu}}\right)-\hbar \omega^{1} \Gamma_{\mu}^{\prime}(\varphi(\hat{x})),
$$

Por esta equação, vemos que $Q_{\omega}\left(p_{\alpha} \frac{\partial x^{\alpha}}{\partial x^{\prime \mu}}\right)$ é um operador auto-adjunto se e somente se $\omega^{1}$ é real. Este é evidentemente o caso se $Q_{\omega}$ é auto-adjunta (ou seja, quando se verifica (2.29)). Assim, a equação (1.9) não é possível para qualquer quantização $Q_{\omega}$, pois $\hat{p}_{\mu}^{\prime}$ deve ser auto-adjunto. Vamos assumir a seguir que $Q_{\omega}$ é auto-adjunta. Usando mais uma vez a expressão (2.57), temos

$$
Q_{\omega}\left(p_{\alpha} \frac{\partial x^{\alpha}}{\partial x^{\prime \mu}}\right)=\frac{\widehat{\partial x^{\alpha}}}{\partial x^{\prime \mu}} \hat{p}_{\alpha}-\hbar\left(\frac{i}{2}+\omega^{1}\right) \Gamma_{\mu}^{\prime}(\varphi(\hat{x}))
$$


Por (2.53), temos

$$
\begin{aligned}
& U \frac{\widehat{\partial x^{\alpha}}}{\partial x^{\prime \mu}} U^{\dagger}=U \frac{\partial x^{\alpha}}{\partial x^{\prime \mu}}(\varphi(\hat{x})) U^{\dagger}=\frac{\partial x^{\alpha}}{\partial x^{\prime \mu}}\left(\hat{x}^{\prime}\right) \\
& U \Gamma_{\mu}^{\prime}(\varphi(\hat{x})) U^{\dagger}=\Gamma_{\mu}^{\prime}\left(\hat{x}^{\prime}\right) .
\end{aligned}
$$

A equação (1.9) fica então

$$
\hat{p}_{\mu}^{\prime}=\frac{\partial x^{\alpha}}{\partial x^{\prime \mu}}\left(\hat{x}^{\prime}\right) U \hat{p}_{\alpha} U^{\dagger}-\hbar\left(\frac{i}{2}+\omega^{1}\right) \Gamma_{\mu}^{\prime}\left(\hat{x}^{\prime}\right) .
$$

As equações (2.53) e (2.58) definem $U$ a menos de uma fase global. Sejam $|x\rangle$ em $\mathcal{R}$ os auto-vetores da posição definidos por (2.46). Podemos então definir no espaço de Hilbert $\mathcal{R}^{\prime}$ os vetores $\left|x^{\prime}\right\rangle^{\prime}=|\varphi(x)\rangle^{\prime}{ }^{5}$ por

$$
|\varphi(x)\rangle^{\prime}=U|x\rangle
$$

Usaremos a notação $\left\langle\left. x^{\prime}\right|^{\prime} \text { para o funcional associado a } \mid x^{\prime}\right\rangle^{\prime}$. A equação (2.53) mostra que eles são auto-estados de $\hat{x}^{\prime \mu}$ :

$$
\begin{gathered}
\hat{x}^{\prime \mu}|\varphi(x)\rangle^{\prime}=U \varphi^{\mu}(\hat{x}) U^{\dagger}|\varphi(x)\rangle^{\prime}=U \varphi^{\mu}(\hat{x})|x\rangle=\varphi^{\mu}(x) U|x\rangle=\varphi^{\mu}(x)|\varphi(x)\rangle^{\prime} \\
\left(U^{\dagger}=U^{-1}\right), \text { ou } \\
\hat{x}^{\prime \mu}\left|x^{\prime}\right\rangle^{\prime}=x^{\prime \mu}\left|x^{\prime}\right\rangle^{\prime} .
\end{gathered}
$$

A normalização surge da unitariedade de $U$ :

$$
\left\langle\left. x^{\prime}\right|^{\prime} y^{\prime}\right\rangle^{\prime}=\left\langle x\left|U^{\dagger} U\right| y\right\rangle=\langle x \mid y\rangle=\delta(x-y)=\frac{\delta\left(x^{\prime}-y^{\prime}\right)}{\left|\operatorname{det}\left(\frac{\partial x}{\partial x^{\prime}}\right)\right|}=\frac{\delta\left(x^{\prime}-y^{\prime}\right)}{\sqrt{g^{\prime}\left(x^{\prime}\right)}},
$$

onde $g^{\prime}$ é o determinante do tensor métrico no sistema de coordenadas $x^{\prime}$, e foi usada a propriedade da função $\delta$ multi-dimensional $\delta\left(f\left(x^{\prime}\right)\right)=\delta\left(x^{\prime}-y^{\prime}\right) /\left|\frac{\partial f}{\partial x^{\prime}}\right|$, onde $f\left(x^{\prime}\right)=\varphi^{-1}\left(x^{\prime}\right)-\varphi^{-1}\left(y^{\prime}\right)\left(f\left(x^{\prime}\right)=0\right.$ tem uma única raiz $x^{\prime}=y^{\prime}$, pois $\varphi$ é um difeomorfismo). Esta normalização implica que a relação de completude é

$$
\int\left|x^{\prime}\right\rangle^{\prime}\left\langle\left. x^{\prime}\right|^{\prime} \sqrt{g^{\prime}\left(x^{\prime}\right)} d x^{\prime}=\hat{I} .\right.
$$

\footnotetext{
${ }^{5}$ Não usaremos auto-estados do momento. Assim, $x \rightarrow|x\rangle$ será sempre uma função que ao vetor $x \in \mathbb{R}^{D}$ associa o auto-estado $|x\rangle \in \mathcal{R}$ de $\hat{x}^{\mu}$ e $x^{\prime} \rightarrow\left|x^{\prime}\right\rangle^{\prime}$ será sempre uma função que associa $x^{\prime} \in \mathbb{R}^{D}$ ao auto-estado $\left|x^{\prime}\right\rangle^{\prime} \in \mathcal{R}^{\prime}$ de $\hat{x}^{\prime \mu}$.
} 
A última propriedade é a forma da representação do operador de momento. Temos

$$
\begin{aligned}
\left\langle\left. x^{\prime}\right|^{\prime} \frac{\partial x^{\alpha}}{\partial x^{\prime \mu}}\right. & \left(\hat{x}^{\prime}\right) U \hat{p}_{\alpha} U^{\dagger}=\frac{\partial x^{\alpha}}{\partial x^{\prime \mu}}\left(x^{\prime}\right)\left\langle\left. x^{\prime}\right|^{\prime} U \hat{p}_{\alpha} U^{\dagger}=\frac{\partial x^{\alpha}}{\partial x^{\prime \mu}}\left(x^{\prime}\right)\langle x| \hat{p}_{\alpha} U^{\dagger}\right. \\
& =-i \hbar \frac{\partial x^{\alpha}}{\partial x^{\prime \mu}}\left(x^{\prime}\right) \frac{\partial}{\partial x^{\alpha}}\langle x| U^{\dagger}=-i \hbar \frac{\partial x^{\alpha}}{\partial x^{\prime \mu}}\left(x^{\prime}\right) \frac{\partial}{\partial x^{\alpha}}\left\langle\left.\varphi(x)\right|^{\prime}=-i \hbar \frac{\partial}{\partial x^{\prime \mu}}\left\langle\left. x^{\prime}\right|^{\prime}\right.\right.
\end{aligned}
$$

Usando (2.58), temos portanto

$$
\left\langle\left. x^{\prime}\right|^{\prime} \hat{p}_{\mu}^{\prime}=-i \hbar\left(\frac{\partial}{\partial x^{\prime \mu}}+\left(\frac{1}{2}-i \omega^{1}\right) \Gamma_{\mu}^{\prime}\left(x^{\prime}\right)\right)\left\langle\left. x^{\prime}\right|^{\prime} .\right.\right.
$$

Dado algum estado $|\psi\rangle \in \mathcal{R}$, seu correspondente em $\mathcal{R}^{\prime}$ é $|\psi\rangle^{\prime}=U|\psi\rangle$. A definição (2.59) corresponde à escolha de uma representação de $\mathcal{R}^{\prime}$ em que as funções de onda são escalares:

$$
\psi^{\prime}\left(x^{\prime}\right)=\left\langle\left. x^{\prime}\right|^{\prime} \psi\right\rangle^{\prime}=\left\langle x\left|U^{\dagger} U\right| \psi\right\rangle=\langle x \mid \psi\rangle=\psi(x)
$$

Podemos agora calcular diretamente $Q_{\omega}^{\prime}$ pela definição $(2.52)$ e pela forma $(2.48)$ de $Q_{\omega}(F)$ :

$$
Q_{\omega}^{\prime}\left(F^{\prime}\right)=U Q_{\omega}(F) U^{\dagger}=\int \tilde{F}(x, \xi) U \hat{D}(x, \xi, \omega) U^{\dagger} d x d \xi
$$

Gostaríamos de executar a mudança de variáveis $x^{\prime}=\varphi(x), \xi^{\prime}=\frac{\partial x^{\prime}}{\partial x} \xi$,

$$
d x d \xi=\left|\operatorname{det}\left(\frac{\partial x}{\partial x^{\prime}}\right)\right|^{2} d x^{\prime} d \xi^{\prime}=g^{\prime}\left(x^{\prime}\right) d x^{\prime} d \xi^{\prime},
$$

no integrando de (2.63). No sistema de coordenadas $x, g_{\mu \nu}$ é dado por (2.17) e portanto $g=1$. Assim, sob esta mudança, a transformação de Fourier (2.41) é dada por (2.43) como

$$
\tilde{F}(x, \xi)=\frac{\tilde{F}^{\prime}\left(x^{\prime}, \xi^{\prime}\right)}{\sqrt{g^{\prime}\left(x^{\prime}\right)}}
$$

onde $g^{\prime}=\left|\operatorname{det}\left(g_{\mu \nu}^{\prime}\right)\right|$ e $g_{\mu \nu}^{\prime}$ é a métrica no sistema de coordenadas $x^{\prime}$. Usando as definições (2.49) e (2.59), podemos calcular o operador do integrando em (2.63):

$$
\begin{aligned}
U \hat{D}(x, \xi, \omega) U^{\dagger} & =\int U\left|x-\left(\frac{1}{2}+\theta\right) \xi\right\rangle\left\langle x+\left(\frac{1}{2}-\theta\right) \xi\right| U^{\dagger} \omega(\theta) d \theta \\
& =\int\left|\varphi\left(x-\left(\frac{1}{2}+\theta\right) \xi\right)\right\rangle^{\prime}\left\langle\left.\varphi\left(x+\left(\frac{1}{2}-\theta\right) \xi\right)\right|^{\prime} \omega(\theta) d \theta\right.
\end{aligned}
$$

Usando a definição da função exponencial $\gamma^{\prime}$ no sistema de coordenadas $x^{\prime}$ (ver 
apêndice C, fórmula (C.17) no caso particular (C.19)),

$$
\begin{aligned}
U \hat{D}(x, \xi, \omega) U^{\dagger} & =\hat{D}^{\prime}\left(x^{\prime}, \xi^{\prime}, \omega\right) \\
& =\int\left|\gamma^{\prime}\left(x^{\prime},-\left(\frac{1}{2}+\theta\right) \xi^{\prime}\right)\right\rangle^{\prime}\left\langle\left.\gamma^{\prime}\left(x^{\prime},\left(\frac{1}{2}-\theta\right) \xi^{\prime}\right)\right|^{\prime} \omega(\theta) d \theta\right.
\end{aligned}
$$

Assim, a aplicação da mudança de variáveis sobre a integral (2.63) dá

$$
Q_{\omega}^{\prime}\left(F^{\prime}\right)=\int \tilde{F}^{\prime}\left(x^{\prime}, \xi^{\prime}\right) \hat{D}^{\prime}\left(x^{\prime}, \xi^{\prime}, \omega\right) \sqrt{g^{\prime}\left(x^{\prime}\right)} d x^{\prime} d \xi^{\prime}
$$

Esta expressão mostra que a definição de $Q_{\omega}^{\prime}\left(F^{\prime}\right)$ por meio de (2.52) é independente do sistema de coordenadas original $x$, pois se expressa unicamente em termos do determinante da métrica $g^{\prime}$ e da função exponencial, definida em termos da conexão de Levi-Civita $\Gamma_{\alpha \beta}^{\prime \mu}$ no referencial $x^{\prime}$.

A quantização $Q_{\omega}^{\prime}$ em coordenadas gerais, como definida por (2.52), é automaticamente covariante. De fato, seja $x^{\prime \prime}=\zeta(x)$ outro referencial geral e $\mathcal{R}^{\prime \prime}$ o espaço de Hilbert para a quantização nestas coordenadas. A transformação linear $V: \mathcal{R} \rightarrow \mathcal{R}^{\prime \prime}$ que relaciona a quantização no sistema de coordenadas ortonormais $x$ à quantização em $x^{\prime \prime}$ obedece a $Q_{\omega}^{\prime \prime}\left(F^{\prime \prime}\right)=V Q_{\omega}(F) V^{\dagger}$. Assim, dada a unitariedade de $U$, é claro que $Q_{\omega}(F)=U^{\dagger} Q_{\omega}^{\prime}\left(F^{\prime}\right) U$ e, portanto, $Q_{\omega}^{\prime \prime}\left(F^{\prime \prime}\right)=W Q_{\omega}^{\prime}\left(F^{\prime}\right) W^{\dagger}$, com $W=V U^{\dagger}$ unitária.

O limite clássico também existe em coordenadas gerais $x^{\prime}$. De fato, se $x$ é um sistema de coordenadas euclidianas ou pseudo-euclidianas, e $*$ e $*^{\prime}$ são os produtos de símbolos relativos às quantizações $Q_{\omega}$ e $Q_{\omega}^{\prime}$, então

$$
\begin{aligned}
Q_{\omega}^{\prime}\left(F^{\prime} *^{\prime} G^{\prime}\right) & =Q_{\omega}^{\prime}\left(F^{\prime}\right) Q_{\omega}^{\prime}\left(G^{\prime}\right)=U^{\dagger} Q_{\omega}(F) Q_{\omega}(G) U \\
& =U^{\dagger} Q_{\omega}(F * G) U=Q_{\omega}^{\prime}\left((F * G)^{\prime}\right)
\end{aligned}
$$

e, portanto,

$$
\begin{aligned}
F^{\prime} *^{\prime} G^{\prime}= & (F * G)^{\prime}=(F G+O(\hbar))^{\prime}=F^{\prime} G^{\prime}+O(\hbar) \\
F^{\prime} *^{\prime} G^{\prime}-G^{\prime} *^{\prime} F^{\prime} & =(F * G)^{\prime}-(G * F)^{\prime} \\
& =\left(i \hbar\{F, G\}+O\left(\hbar^{2}\right)\right)^{\prime}=i \hbar\left\{F^{\prime}, G^{\prime}\right\}^{\prime}+O\left(\hbar^{2}\right),
\end{aligned}
$$

onde $\{$,$\} e \{,\}^{\prime}$ são os parenteses de Poisson em coordenadas $(x, p)$ e $\left(x^{\prime}, p^{\prime}\right)$ respectivamente. A igualdade $\{F, G\}=\left\{F^{\prime}, G^{\prime}\right\}^{\prime}$ vem do fato de que as transformações de ponto $(1.5,1.6)$ são transformações canônicas independentes do tempo. 
Vamos sistematizar a definição de $Q_{\omega}$ em um sistema de coordenadas geral $x$, sem fazer referência a um sistema de coordenadas euclidianas. Consideremos um espaço plano (isto é, existe um sistema de coordenadas em que a métrica é (2.17)) onde o tensor métrico é $g_{\mu \nu}(x)$ e portanto há uma conexão de Levi-Civita $\Gamma_{\alpha \beta}^{\mu}(x)$, dada por (B.1). Esta conexão define a função exponencial $\gamma(x, \xi)$. Vamos definir um espaço de Hilbert $\mathcal{R}$ onde atuam os operadores usuais $\hat{x}^{\mu}, \hat{p}_{\mu}$ e neste espaço definir uma base $|x\rangle_{\omega^{1}}$ de auto-vetores de $\hat{x}^{\mu}$ obedecendo a

$$
\begin{gathered}
\hat{x}^{\mu}|x\rangle_{\omega^{1}}=x^{\mu}|x\rangle_{\omega^{1}},\left\langle\left. x\right|_{\omega^{1}} y\right\rangle_{\omega^{1}}=\frac{\delta(x-y)}{\sqrt{g(x)}}, \\
\left\langle\left. x\right|_{\omega^{1}} \hat{p}_{\mu}=-i \hbar\left(\frac{\partial}{\partial x^{\mu}}+\left(\frac{1}{2}-i \omega^{1}\right) \Gamma_{\mu}(x)\right)\left\langle\left. x\right|_{\omega^{1}} .\right.\right.
\end{gathered}
$$

Então definimos o operador em $\mathcal{R}$

$$
\hat{D}(x, \xi, \omega)=\int\left|\gamma\left(x,-\left(\frac{1}{2}+\theta\right) \xi\right)\right\rangle_{\omega^{1}}\left\langle\left.\gamma\left(x,\left(\frac{1}{2}-\theta\right) \xi\right)\right|_{\omega^{1}} \omega(\theta) d \theta .\right.
$$

Através deste operador definimos a correspondência

$$
Q_{\omega}(F)=\int \tilde{F}(x, \xi) \hat{D}(x, \xi, \omega) \sqrt{g(x)} d x d \xi
$$

Quando $F(x, p)$ é polinomial em $p$, temos, devido a (2.50) e analogamente a (2.51),

$$
Q_{\omega}(F)=\left.\int F\left(x,-i \hbar \frac{\partial}{\partial \xi}\right) \hat{D}(x, \xi, \omega)\right|_{\xi=0} \sqrt{g(x)} d x .
$$

Quando não houver ambigüidade, omitiremos o índice $\omega^{1}$ nos vetores |\rangle$_{\omega^{1}}$. O vetor $|x\rangle_{\omega^{1}}$ se relaciona com o vetor $|x\rangle_{0}$ por

$$
|x\rangle_{\omega^{1}}=e^{-i \omega^{1} \ln \sqrt{g(x)}}|x\rangle_{0}
$$

o que pode ser verificado, considerando a igualdade (B.2), pelo cálculo

$$
\begin{aligned}
e^{i \omega^{1} \ln \sqrt{g(x)}}\left\langle\left. x\right|_{0} \hat{p}_{\mu}\right. & =e^{i \omega^{1} \ln \sqrt{g(x)}}(-i \hbar)\left(\frac{\partial}{\partial x^{\mu}}+\frac{1}{2} \Gamma_{\mu}(x)\right)\left\langle\left. x\right|_{0}\right. \\
& =-i \hbar\left(e^{i \omega^{1} \ln \sqrt{g(x)}} \frac{\partial}{\partial x^{\mu}} e^{-i \omega^{1} \ln \sqrt{g(x)}}+\frac{1}{2} \Gamma_{\mu}(x)\right) e^{i \omega^{1} \ln \sqrt{g(x)}}\left\langle\left. x\right|_{0}\right. \\
& =-i \hbar\left(\frac{\partial}{\partial x^{\mu}}+\left(\frac{1}{2}-i \omega^{1}\right) \Gamma_{\mu}(x)\right) e^{i \omega^{1} \ln \sqrt{g(x)}}\left\langle\left. x\right|_{0}\right.
\end{aligned}
$$




\subsection{Representação da posição em coordenadas ge- rais e exemplos de operadores}

Os auto-estados da posição definidos por (2.64) e (2.65), necessários para definir o operador (2.66), definem uma representação da posição no espaço de Hilbert $\mathcal{R}$ segundo a qual as funções de onda são escalares pela transformação geral $x^{\prime}=$ $\varphi(x)$, como mostra a equação (2.62). A representação da posição $\left(Q_{\omega}(F)\right)_{x}$ de um operador $Q_{\omega}(F)$ será portanto um operador escalar, no sentido de que

$$
\begin{aligned}
\left(Q_{\omega}^{\prime}\left(F^{\prime}\right)\right)_{x^{\prime}} \psi^{\prime}\left(x^{\prime}\right) & \equiv\left\langle x^{\prime}\left|Q_{\omega}^{\prime}\left(F^{\prime}\right)\right| \psi\right\rangle^{\prime}= \\
\left\langle x\left|U^{\dagger} Q_{\omega}^{\prime}\left(F^{\prime}\right) U\right| \psi\right\rangle & =\left\langle x\left|Q_{\omega}(F)\right| \psi\right\rangle \equiv\left(Q_{\omega}(F)\right)_{x} \psi(x) .
\end{aligned}
$$

Vamos usar este fato para calcular a representação da posição do operador $Q_{\omega}(F)$ correspondente a um polinômio homogêneo $F(x, p)=T^{\mu_{1} \cdots \mu_{n}}(x) p_{\mu_{1}} \cdots p_{\mu_{n}}$, com $T^{\mu_{1} \cdots \mu_{n}}$ completamente simétrico em todos os índices. Pela equação geral (2.65), o operador de momento em coordenadas retilíneas $\left(\Gamma_{\alpha \beta}^{\mu}=0\right)$ é $\left(\hat{p}_{\mu}\right)_{x}=-i \hbar \frac{\partial}{\partial x^{\mu}}$. Substituindo este operador na expressão de $Q_{\omega}(F)$ em coordenadas retilíneas dada por (2.34), obtemos

$$
\begin{aligned}
& \left(Q_{\omega}\left(T^{\mu_{1} \cdots \mu_{n}}(x) p_{\mu_{1}} \cdots p_{\mu_{n}}\right)\right)_{x}= \\
& \quad(-i \hbar)^{n} \sum_{k=0}^{n} \omega_{k}^{n} \frac{\partial}{\partial x^{\mu_{1}}} \cdots \frac{\partial}{\partial x^{\mu_{k}}} T^{\mu_{1} \cdots \mu_{n}}(x) \frac{\partial}{\partial x^{\mu_{k+1}}} \cdots \frac{\partial}{\partial x^{\mu_{n}}} .
\end{aligned}
$$

A expressão escalar para este operador, que coincide com a expressão acima em coordenadas retilíneas, obtém-se pela substituição $\frac{\partial}{\partial x^{\mu}} \rightarrow \nabla_{\mu}$ de derivadas comuns por derivadas covariantes. Assim, a representação da posição de $Q_{\omega}$ em coordenadas gerais, para polinômios homogêneos, é

$$
\begin{aligned}
\left(Q_{\omega}\left(T^{\mu_{1} \cdots \mu_{n}}(x) p_{\mu_{1}} \cdots p_{\mu_{n}}\right)\right)_{x} & = \\
& (-i \hbar)^{n} \sum_{k=0}^{n} \omega_{k}^{n} \nabla_{\mu_{1}} \ldots \nabla_{\mu_{k}} T^{\mu_{1} \cdots \mu_{n}}(x) \nabla_{\mu_{k+1}} \ldots \nabla_{\mu_{n}} .
\end{aligned}
$$

A seguir, apresentaremos exemplos de operadores calculados através da fórmula acima e (2.67).

1. Uma função exclusivamente da posição: $F(x, p)=F(x)$. Temos $\tilde{F}(x, \xi)=$ $F(x) \delta(\xi)$. Pela definição (2.66), usando a propriedade $\gamma(x, 0)=x$, vemos 
que

$$
\hat{D}(x, 0, \omega)=|x\rangle\langle x|
$$

Substituindo em (2.67)

$$
Q_{\omega}(F)=\int F(x)|x\rangle\langle x| \sqrt{g(x)} d x=F(\hat{x})
$$

Na representação da posição,

$$
\left(Q_{\omega}(F)\right)_{x}=F(x)
$$

2. Uma função linear no momento, $F(x, p)=T^{\alpha}(x) p_{\alpha}$. Pela fórmula (2.71), usando as equações $(2.54),(2.55)$ e (2.56),

$$
\begin{aligned}
\left(Q_{\omega}\left(T^{\alpha} p_{\alpha}\right)\right)_{x} & =-i \hbar\left[\omega_{0}^{1} T^{\alpha} \nabla_{\alpha}+\omega_{1}^{1} \nabla_{\alpha} T^{\alpha}\right] \\
& =-i \hbar\left[\omega_{0}^{1} T^{\alpha} \frac{\partial}{\partial x^{\alpha}}+\omega_{1}^{1}\left(\frac{\partial}{\partial x^{\alpha}}+\Gamma_{\alpha}\right) T^{\alpha}\right] \\
& =-i \hbar\left[\frac{1}{2}\left(T^{\alpha} \frac{\partial}{\partial x^{\alpha}}+\frac{\partial}{\partial x^{\alpha}} T^{\alpha}+\Gamma_{\alpha} T^{\alpha}\right)-i \omega^{1}\left(\left[\frac{\partial}{\partial x^{\alpha}}, T^{\alpha}\right]+\Gamma_{\alpha} T^{\alpha}\right)\right] \\
& =-i \hbar\left[\frac{1}{2}\left(T^{\alpha} \frac{\partial}{\partial x^{\alpha}}+\frac{\partial}{\partial x^{\alpha}} T^{\alpha}+\Gamma_{\alpha} T^{\alpha}\right)-i \omega^{1}\left(\frac{\partial T^{\alpha}}{\partial x^{\alpha}}+\Gamma_{\alpha} T^{\alpha}\right)\right],
\end{aligned}
$$

O operador de momento pode ser obtido com a escolha $T^{\alpha}=\delta_{\mu}^{\alpha}$,

$$
\left(Q_{\omega}\left(p_{\mu}\right)\right)_{x}=\left(\hat{p}_{\mu}\right)_{x}=-i \hbar\left[\frac{\partial}{\partial x^{\mu}}+\left(\frac{1}{2}-i \omega^{1}\right) \Gamma_{\mu}\right],
$$

consistente com (2.65). A expressão de um operador independente da representação pode então ser obtida pela substituição

$$
\frac{\partial}{\partial x^{\mu}} \rightarrow \frac{i}{\hbar} \hat{p}_{\mu}-\left(\frac{1}{2}-i \omega^{1}\right) \Gamma_{\mu}(\hat{x}), x^{\mu} \rightarrow \hat{x}^{\mu}
$$

Realizando esta substituição na expressão para $\left(Q_{\omega}\left(T^{\alpha} p_{\alpha}\right)\right)_{x}$, obtemos

$$
Q_{\omega}\left(T^{\alpha} p_{\alpha}\right)=\frac{1}{2}\left(T^{\alpha}(\hat{x}) \hat{p}_{\alpha}+\hat{p}_{\alpha} T^{\alpha}(\hat{x})\right)-\hbar \omega^{1} \frac{\partial T^{\alpha}}{\partial x^{\alpha}}(\hat{x})
$$

3. $F(x, p)=g^{\mu \nu}(x) p_{\mu} p_{\nu}=p^{2}$. Como a derivada covariante do tensor métrico é zero, temos $g^{\mu \nu} \nabla_{\mu} \nabla_{\nu}=\nabla_{\mu} \nabla_{\nu} g^{\mu \nu}=\nabla_{\mu} g^{\mu \nu} \nabla_{\nu}=\frac{1}{\sqrt{g}} \frac{\partial}{\partial x^{\mu}} \sqrt{g} g^{\mu \nu} \frac{\partial}{\partial x^{\nu}}$, o operador de Lapace-Beltrami. Levando em conta a propriedade (2.36) dos coeficientes 
$\omega_{k}^{n}$,

$$
\begin{aligned}
\left(Q_{\omega}\left(g^{\mu \nu}(x) p_{\mu} p_{\nu}\right)\right)_{x} & =-\hbar^{2}\left(\omega_{0}^{2}+\omega_{1}^{2}+\omega_{2}^{2}\right) \frac{1}{\sqrt{g}} \frac{\partial}{\partial x^{\mu}} \sqrt{g} g^{\mu \nu} \frac{\partial}{\partial x^{\nu}} \\
& =-\hbar^{2} \frac{1}{\sqrt{g}} \frac{\partial}{\partial x^{\mu}} \sqrt{g} g^{\mu \nu} \frac{\partial}{\partial x^{\nu}},
\end{aligned}
$$

independente da função $\omega(\theta)$. Usando a propriedade

$$
\Gamma_{\mu}=\frac{\partial \ln (\sqrt{g})}{\partial x^{\mu}}
$$

(ver apêndice B), a equação (2.72) pode ser reescrita como

$$
\left(\hat{p}_{\mu}\right)_{x}=-i \hbar g^{-\frac{1}{4}} e^{i \omega^{1} \sqrt{g}} \frac{\partial}{\partial x^{\mu}} g^{\frac{1}{4}} e^{-i \omega^{1} \sqrt{g}}
$$

o que implica a relação inversa

$$
\frac{\partial}{\partial x^{\mu}}=\frac{i}{\hbar} g^{\frac{1}{4}} e^{-i \omega^{1} \sqrt{g}}\left(\hat{p}_{\mu}\right)_{x} g^{-\frac{1}{4}} e^{i \omega^{1} \sqrt{g}}
$$

Substituindo esta expressão em (2.75), obtemos a quantização no espaço de Hilbert abstrato

$$
Q_{\omega}\left(g^{\mu \nu}(x) p_{\mu} p_{\nu}\right)=e^{-i \omega^{1} \sqrt{g}} g^{-\frac{1}{4}} \hat{p}_{\mu} g^{\frac{1}{2}} g^{\mu \nu} \hat{p}_{\nu} g^{-\frac{1}{4}} e^{i \omega^{1} \sqrt{g}}
$$

4. Monômio de grau 2 no momento, $F(p)=p_{\alpha} p_{\beta}=T^{\mu \nu} p_{\mu} p_{\nu}$, com $T^{\mu \nu}=$ $\frac{1}{2}\left(\delta_{\alpha}^{\mu} \delta_{\beta}^{\nu}+\delta_{\beta}^{\mu} \delta_{\alpha}^{\nu}\right)$. Vamos considerar o caso particular em que $\omega(\theta)$ é real, no qual se enquadram os ordenamentos de Weyl e de Born-Jordan. Neste caso, os coeficientes (2.35) obedecem a $\omega_{k}^{n}=\omega_{n-k}^{n}$. Assim,

$$
\begin{aligned}
\left(Q_{\omega}\left(p_{\alpha} p_{\beta}\right)\right)_{x} & =(-i \hbar)^{2}\left(\omega_{0}^{2} T^{\mu \nu} \nabla_{\mu} \nabla_{\nu}+\omega_{1}^{2} \nabla_{\mu} T^{\mu \nu} \nabla_{\nu}+\omega_{2}^{2} \nabla_{\mu} \nabla_{\nu} T^{\mu \nu}\right) \\
& =(-i \hbar)^{2}\left(\omega_{0}^{2}\left(T^{\mu \nu} \nabla_{\mu} \nabla_{\nu}+\nabla_{\mu} \nabla_{\nu} T^{\mu \nu}\right)+\omega_{1}^{2} \nabla_{\mu} T^{\mu \nu} \nabla_{\nu}\right)
\end{aligned}
$$

com $2 \omega_{0}^{2}+\omega_{1}^{2}=1$. Os operadores envolvendo derivadas covariantes se expres- 
sam como

$$
\begin{aligned}
T^{\mu \nu} \nabla_{\mu} \nabla_{\nu}= & T^{\mu \nu}\left(\frac{\partial}{\partial x^{\mu}} \frac{\partial}{\partial x^{\nu}}-\Gamma_{\mu \nu}^{\rho} \frac{\partial}{\partial x^{\rho}}\right)=\frac{\partial}{\partial x^{\alpha}} \frac{\partial}{\partial x^{\beta}}-\Gamma_{\alpha \beta}^{\rho} \frac{\partial}{\partial x^{\rho}} \\
\nabla_{\mu} T^{\mu \nu} \nabla_{\nu}= & \left(\frac{\partial}{\partial x^{\mu}}+\Gamma_{\mu}\right) T^{\mu \nu} \frac{\partial}{\partial x^{\nu}}=\frac{\partial}{\partial x^{\alpha}} \frac{\partial}{\partial x^{\beta}}+\frac{1}{2}\left(\Gamma_{\alpha} \frac{\partial}{\partial x^{\beta}}+\Gamma_{\beta} \frac{\partial}{\partial x^{\alpha}}\right) \\
\nabla_{\mu} \nabla_{\nu} T^{\mu \nu}= & \left(\frac{\partial}{\partial x^{\mu}}+\Gamma_{\mu}\right)\left(\frac{\partial}{\partial x^{\nu}} T^{\mu \nu}+\Gamma_{\nu \rho}^{\mu} T^{\rho \nu}+\Gamma_{\rho} T^{\mu \rho}\right) \\
= & \frac{\partial}{\partial x^{\alpha}} \frac{\partial}{\partial x^{\beta}}+\frac{\partial}{\partial x^{\rho}} \Gamma_{\alpha \beta}^{\rho}+\Gamma_{\rho} \Gamma_{\alpha \beta}^{\rho}+\Gamma_{\alpha} \Gamma_{\beta} \\
& +\frac{\partial \Gamma_{\alpha}}{\partial x^{\beta}}+\left(\Gamma_{\alpha} \frac{\partial}{\partial x^{\beta}}+\Gamma_{\beta} \frac{\partial}{\partial x^{\alpha}}\right) .
\end{aligned}
$$

(para o cálculo do último operador, é necessário levar em conta que $\frac{\partial \Gamma_{\alpha}}{\partial x^{\beta}}=\frac{\partial \Gamma_{\beta}}{\partial x^{\alpha}}$, pois $\left.\Gamma_{\alpha}=\frac{\partial \ln \sqrt{g}}{\partial x^{\alpha}}\right)$. Substituindo na expressão para $\left(Q_{\omega}\left(p_{\alpha} p_{\beta}\right)\right)_{x}$,

$$
\begin{aligned}
\left(Q_{\omega}\left(p_{\alpha} p_{\beta}\right)\right)_{x}=-\hbar^{2}\left(\frac{\partial}{\partial x^{\alpha}} \frac{\partial}{\partial x^{\beta}}\right. & +\frac{1}{2}\left(\Gamma_{\alpha} \frac{\partial}{\partial x^{\beta}}+\Gamma_{\beta} \frac{\partial}{\partial x^{\alpha}}\right) \\
& \left.+\omega_{0}^{2}\left(\frac{\partial \Gamma_{\alpha}}{\partial x^{\beta}}+\frac{\partial \Gamma_{\alpha \beta}^{\rho}}{\partial x^{\rho}}+\Gamma_{\rho} \Gamma_{\alpha \beta}^{\rho}+\Gamma_{\alpha} \Gamma_{\beta}\right)\right) .
\end{aligned}
$$

Como estamos usando $\omega(\theta)$ real, temos $\omega^{1}=0$. Assim, para obter o operador acima independente da representação, devemos efetuar a subtstituição (2.73) com $\omega^{1}=0$, obtendo

$Q_{\omega}\left(p_{\alpha} p_{\beta}\right)=\hat{p}_{\alpha} \hat{p}_{\beta}-\hbar^{2}\left(-\frac{1}{2} \frac{\partial \Gamma_{\alpha}}{\partial x^{\beta}}-\frac{1}{4} \Gamma_{\alpha} \Gamma_{\beta}+\omega_{0}^{2}\left(\frac{\partial \Gamma_{\alpha}}{\partial x^{\beta}}+\frac{\partial \Gamma_{\alpha \beta}^{\rho}}{\partial x^{\rho}}+\Gamma_{\rho} \Gamma_{\alpha \beta}^{\rho}+\Gamma_{\alpha} \Gamma_{\beta}\right)\right)$.

No caso do ordenamento de Weyl, $\omega_{0}^{2}=\frac{1}{4}$, e a expressão acima toma a forma particularmente simples

$$
Q^{\mathrm{Weyl}}\left(p_{\alpha} p_{\beta}\right)=\hat{p}_{\alpha} \hat{p}_{\beta}-\frac{\hbar^{2}}{4}\left(R_{\alpha \beta}+\Gamma_{\alpha \sigma}^{\rho} \Gamma_{\beta \rho}^{\sigma}\right)=\hat{p}_{\alpha} \hat{p}_{\beta}-\frac{\hbar^{2}}{4} \Gamma_{\alpha \sigma}^{\rho} \Gamma_{\beta \rho}^{\sigma},
$$

onde $R_{\alpha \beta}=\frac{\partial \Gamma_{\alpha \beta}^{\rho}}{\partial x^{\rho}}-\frac{\partial \Gamma_{\alpha}}{\partial x^{\beta}}+\Gamma_{\rho} \Gamma_{\alpha \beta}^{\rho}-\Gamma_{\alpha \sigma}^{\rho} \Gamma_{\beta \rho}^{\sigma}$ é o tensor de Ricci, que é nulo quando o espaço é plano. 


\section{Capítulo 3}

\section{Generalização de $Q_{\omega}$ a espaços}

\section{curvos}

Consideraremos agora um espaço de Riemann curvo, isto é, um espaço em que esteja definido um tensor métrico $g_{\mu \nu}(x)$ em coordenadas $x$ que não pode ser reduzido à forma constante (2.17) por mudança de coordenadas. Vamos admitir que o espaço todo pode ser coberto por um único sistema de coordenadas. As definições (2.64), (2.65), (2.66) e (2.67) aplicam-se de forma direta a um espaço curvo, usando a conexão de Levi-Civita $\Gamma_{\alpha \beta}^{\mu}(x)$.

É possível generalizar a quantização (2.67) de duas maneiras em um espaço com curvatura.

\subsection{Primeira generalização de $Q_{\omega}$ em espaços cur- $\operatorname{vos}$}

É imediato verificar que a fórmula (2.67) está definida também em um espaço com métrica qualquer (a função exponencial está definida em qualquer espaço de Riemann). Assim, definiremos a primeira generalização de $Q_{\omega}$ em um espaço de Riemann geral como ${ }^{1}$.

$$
Q_{\omega, 1}(F)=\int \tilde{F}(x, \xi) \hat{D}(x, \xi, \omega) \sqrt{g(x)} d x d \xi,
$$

onde o operador $\hat{D}(x, \xi, \omega)$ está definido por (2.66), (2.64) e (2.65).

Das condições enumeradas na seção 1.1, é imediato ver que (3.1) é linear. Pode-

\footnotetext{
${ }^{1}$ Adiante, o índice 1 de $Q_{\omega, 1}$ será interpretado como uma função constante $\mathcal{F}(x, \xi)=1$.
} 
mos calcular o conjugado auto-adjunto do operador $\hat{D}(x, \xi, \omega)$

$$
\begin{aligned}
(\hat{D}(x, \xi, \omega))^{\dagger} & =\int\left|\gamma\left(x,\left(\frac{1}{2}-\theta\right) \xi\right)\right\rangle\left\langle\gamma\left(x,-\left(\frac{1}{2}+\theta\right) \xi\right)\right|(\omega(\theta))^{*} d \theta \\
& =\int\left|\gamma\left(x,\left(\frac{1}{2}+\theta\right) \xi\right)\right\rangle\left\langle\gamma\left(x,-\left(\frac{1}{2}-\theta\right) \xi\right)\right|(\omega(-\theta))^{*} d \theta \\
& =\hat{D}\left(x,-\xi, \omega^{\prime}\right),
\end{aligned}
$$

onde $\omega^{\prime}(\theta)=(\omega(-\theta))^{*}$. Assim, se $\omega$ satisfaz a condição $(2.29), \operatorname{temos}(\hat{D}(x, \xi, \omega))^{\dagger}=$ $\hat{D}(x,-\xi, \omega)$ e, portanto, pela definição (2.41),

$$
\begin{aligned}
\left(Q_{\omega, 1}(F)\right)^{\dagger} & =\int(\tilde{F}(x, \xi))^{*} \hat{D}(x,-\xi, \omega) \sqrt{g(x)} d x d \xi \\
& =\int \widetilde{F^{*}}(x,-\xi) \hat{D}(x,-\xi, \omega) \sqrt{g(x)} d x d \xi \\
& =\int \widetilde{F^{*}}(x, \xi) \hat{D}(x, \xi, \omega) \sqrt{g(x)} d x d \xi \\
& =Q_{\omega, 1}\left(F^{*}\right),
\end{aligned}
$$

ou seja, $Q_{\omega, 1}$ é auto-adjunta.

Repete-se a demonstração, dada no caso de um espaço plano em coordenadas gerais, de que a uma função $F(x)$ somente da variável $x$ corresponde o operador $Q_{\omega, 1}(F)=F(\hat{x})$. Assim, verificam-se as propriedades $Q_{\omega, 1}(1)=\hat{I}$ e $Q_{\omega, 1}\left(x^{\mu}\right)=\hat{x}^{\mu}$. Para demonstrar que $Q_{\omega, 1}\left(p_{\mu}\right)=\hat{p}_{\mu}$, demonstraremos que em um espaço curvo ainda vale a fórmula (2.71). Evidentemente, a demonstração dada para esta fórmula no caso de um espaço plano em coordenadas gerais não vale para um espaço curvo, pois supõe a existência de um sistema de coordenadas retilíneas. Seja então $F(x, p)=$ $T^{\mu_{1} \cdots \mu_{n}}(x) p_{\mu_{1}} \cdots p_{\mu_{n}}$ um polinômio homogêneo na variável $p$, com $T^{\mu_{1} \cdots \mu_{n}}$ simétrico em todos os índices. Vamos usar a definição (2.51). Temos

$$
F\left(x,-i \hbar \frac{\partial}{\partial \xi}\right)=(-i \hbar)^{n} T^{\mu_{1} \cdots \mu_{n}}(x) \frac{\partial}{\partial \xi^{\mu_{1}}} \cdots \frac{\partial}{\partial \xi^{\mu_{n}}} .
$$

Precisamos calcular as derivadas de $\hat{D}(x, \xi, \omega)$ em relação a $\xi$. Pelo resultado do 
apêndice D,

$$
\begin{aligned}
&(-i \hbar)^{n} T^{\mu_{1} \cdots \mu_{n}} \frac{\partial}{\partial \xi^{\mu_{1}}} \cdots \frac{\partial}{\partial \xi^{\mu_{n}}}\left|\gamma\left(x,-\left(\frac{1}{2}+\theta\right) \xi\right)\right\rangle\left\langle\gamma\left(x,\left(\frac{1}{2}-\theta\right) \xi\right)\right|= \\
&(-i \hbar)^{n} T^{\mu_{1} \cdots \mu_{n}} \sum_{k=0}^{n}\left(\begin{array}{l}
n \\
k
\end{array}\right)\left(\frac{\partial}{\partial \xi^{\mu_{1}}} \cdots \frac{\partial}{\partial \xi^{\mu_{k}}}\left|\gamma\left(x,-\left(\frac{1}{2}+\theta\right) \xi\right)\right\rangle\right) \times \\
& \times\left(\frac{\partial}{\partial \xi^{\mu_{k+1}}} \cdots \frac{\partial}{\partial \xi^{\mu_{n}}}\left\langle\gamma\left(x,\left(\frac{1}{2}-\theta\right) \xi\right)\right|\right) .
\end{aligned}
$$

Usando a fórmula (C.21) do apêndice C, temos

$$
\begin{aligned}
\left.\frac{\partial}{\partial \xi^{\mu_{1}}} \cdots \frac{\partial}{\partial \xi^{\mu_{k}}}\left|\gamma\left(x,-\left(\frac{1}{2}+\theta\right) \xi\right)\right\rangle\right|_{\xi=0} & =\left.\left(-\left(\frac{1}{2}+\theta\right)\right)^{k} \frac{\partial}{\partial \xi^{\mu_{1}}} \cdots \frac{\partial}{\partial \xi^{\mu_{k}}}|\gamma(x, \xi)\rangle\right|_{\xi=0} \\
& =(-1)^{k}\left(\frac{1}{2}+\theta\right)^{k} \nabla_{\left(\mu_{1}\right.} \ldots \nabla_{\left.\mu_{k}\right)}|x\rangle \\
\frac{\partial}{\partial \xi^{\mu_{k+1}}} \cdots \frac{\partial}{\partial \xi^{\mu_{n}}}\left\langle\left.\gamma\left(x,\left(\frac{1}{2}-\theta\right) \xi\right)\right|_{\xi=0}\right. & =\left(\frac{1}{2}-\theta\right)^{n-k} \nabla_{\left(\mu_{k+1}\right.} \ldots \nabla_{\left.\mu_{n}\right)}\langle x|
\end{aligned}
$$

e, portanto, usando a definição (2.33) dos coeficientes $C_{k}^{n}(\theta)$, o segundo membro da equação (3.2) fica

$$
(-i \hbar)^{n} T^{\mu_{1} \cdots \mu_{n}} \sum_{k=0}^{n}(-1)^{k} C_{k}^{n}(\theta)\left(\nabla_{\mu_{1}} \ldots \nabla_{\mu_{k}}|x\rangle\right)\left(\nabla_{\mu_{k+1}} \ldots \nabla_{\mu_{n}}\langle x|\right)
$$

onde foram eliminadas as simetrizações dos índices das derivadas covariantes, pois estão contraídas com $T^{\mu_{1} \cdots \mu_{n}}$ que é totalmente simétrico. Com este resultado, usando a definição (2.35) dos coeficientes $\omega_{k}^{n}$, podemos calcular

$$
\begin{aligned}
(-i \hbar)^{n} T^{\mu_{1} \cdots \mu_{n}} & \left.\frac{\partial}{\partial \xi^{\mu_{1}}} \cdots \frac{\partial}{\partial \xi^{\mu_{n}}} \hat{D}(x, \xi, \omega)\right|_{\xi=0}= \\
& (-i \hbar)^{n} T^{\mu_{1} \cdots \mu_{n}} \sum_{k=0}^{n}(-1)^{k} \omega_{k}^{n}\left(\nabla_{\mu_{1}} \ldots \nabla_{\mu_{k}}|x\rangle\right)\left(\nabla_{\mu_{k+1}} \ldots \nabla_{\mu_{n}}\langle x|\right) .
\end{aligned}
$$

Substituindo na definição (2.68),

$$
\begin{aligned}
Q_{\omega, 1}(F)=(-i \hbar)^{n} & \sum_{k=0}^{n}(-1)^{k} \omega_{k}^{n} \times \\
& \times \int T^{\mu_{1} \cdots \mu_{n}}(x)\left(\nabla_{\mu_{1}} \ldots \nabla_{\mu_{k}}|x\rangle\right)\left(\nabla_{\mu_{k+1}} \ldots \nabla_{\mu_{n}}\langle x|\right) \sqrt{g(x)} d x .
\end{aligned}
$$


A representação da posição é então

$$
\begin{aligned}
& \left(Q_{\omega, 1}(F)\right)_{y} \psi(y)=\left\langle y\left|Q_{\omega, 1}(F)\right| \psi\right\rangle=(-i \hbar)^{n} \sum_{k=0}^{n}(-1)^{k} \omega_{k}^{n} \times \\
& \quad \times \int T^{\mu_{1} \cdots \mu_{n}}(x)\left(\nabla_{\mu_{1}} \ldots \nabla_{\mu_{k}} \frac{\delta(x-y)}{\sqrt{g(x)}}\right)\left(\nabla_{\mu_{k+1}} \ldots \nabla_{\mu_{n}} \psi(x)\right) \sqrt{g(x)} d x .
\end{aligned}
$$

O integrando desta última equação pode ser escrito como

$$
\begin{aligned}
\nabla_{\mu_{1}}\left(\left(\nabla_{\mu_{2}} \ldots \nabla_{\mu_{k}} \frac{\delta(x-y)}{\sqrt{g(x)}}\right) T^{\mu_{1} \cdots \mu_{n}}(x) \nabla_{\mu_{k+1}} \ldots \nabla_{\mu_{n}} \psi(x)\right) \\
-\left(\nabla_{\mu_{2}} \ldots \nabla_{\mu_{k}} \frac{\delta(x-y)}{\sqrt{g(x)}}\right) \nabla_{\mu_{1}} T^{\mu_{1} \cdots \mu_{n}}(x) \nabla_{\mu_{k+1}} \ldots \nabla_{\mu_{n}} \psi(x) .
\end{aligned}
$$

O primeiro termo é uma divergência covariante que se anula no infinito (por causa da função $\delta$ ). Assim, sua integral no espaço todo é nula (ver apêndice B). Esta integração por partes pode ser feita sobre todas as derivadas covariantes da função $\delta(x-y) / \sqrt{g(x)}$, surgindo um sinal $(-1)^{j}$. Assim,

$$
\left(Q_{\omega, 1}(F)\right)_{y} \psi(y)=(-i \hbar)^{n} \sum_{k=0}^{n} \omega_{k}^{n} \nabla_{\mu_{1}} \ldots \nabla_{\mu_{k}} T^{\mu_{1} \cdots \mu_{n}}(y) \nabla_{\mu_{k+1}} \ldots \nabla_{\mu_{n}} \psi(y)
$$

Portanto, o operador $Q_{\omega, 1}(F)=Q_{\omega, 1}\left(T^{\mu_{1} \cdots \mu_{n}}(x) p_{\mu_{1}} \cdots p_{\mu_{n}}\right)$ em um espaço curvo na representação da posição coincide com (2.71):

$$
\begin{aligned}
&\left(Q_{\omega, 1}\left(T^{\mu_{1} \cdots \mu_{n}}(x) p_{\mu_{1}} \cdots p_{\mu_{n}}\right)\right)_{x}= \\
&(-i \hbar)^{n} \sum_{k=0}^{n} \omega_{k}^{n} \nabla_{\mu_{1}} \ldots \nabla_{\mu_{k}} T^{\mu_{1} \cdots \mu_{n}}(x) \nabla_{\mu_{k+1}} \ldots \nabla_{\mu_{n}} .
\end{aligned}
$$

Assim, verifica-se o resultado (2.72), o que implica, por comparação com a definição (2.65), que $Q_{\omega, 1}\left(p_{\mu}\right)=\hat{p}_{\mu}$.

Das propriedades da seção (1.1), resta ainda a demonstração da existência do limite clássico. Daremos esta demonstração depois. 


\subsection{Covariância de $Q_{\omega, 1}$ em espaços curvos}

Vamos considerar uma mudança de coordenadas $x^{\prime}=\varphi(x)$. Devido à propriedade $Q_{\omega, 1}(F(x))=F(\hat{x})$, a equação (1.8) permanece igual a (2.53),

$$
\hat{x}^{\prime \mu}=U \varphi^{\mu}(\hat{x}) U^{\dagger}
$$

O resultado (2.74) depende apenas da fórmula (2.71) e da definição (2.65), logo continua válido em um espaço curvo. Pelas relações de comutação entre $\hat{x}^{\mu}$ e $\hat{p}_{\mu}$, ela se escreve como

$$
\begin{aligned}
Q_{\omega, 1}\left(T^{\alpha} p_{\alpha}\right) & =T^{\alpha}(\hat{x}) \hat{p}_{\alpha}+\frac{1}{2}\left[\hat{p}_{\alpha}, T^{\alpha}(\hat{x})\right]-\hbar \omega^{1} \frac{\partial T^{\alpha}}{\partial x^{\alpha}}(\hat{x}) \\
& =T^{\alpha}(\hat{x}) \hat{p}_{\alpha}-i \hbar\left(\frac{1}{2}-i \omega^{1}\right) \frac{\partial T^{\alpha}}{\partial x^{\alpha}}(\hat{x}) .
\end{aligned}
$$

Fazendo $T^{\alpha}=\frac{\partial x^{\alpha}}{\partial x^{\prime \mu}}(\varphi(x))$, temos

$$
\frac{\partial T^{\alpha}}{\partial x^{\alpha}}=\frac{\partial^{2} x^{\alpha}}{\partial x^{\prime \mu} \partial x^{\prime \nu}} \frac{\partial x^{\prime \nu}}{\partial x^{\alpha}}
$$

Podemos então usá-la para calcular

$$
Q_{\omega, 1}\left(p_{\alpha} \frac{\partial x^{\alpha}}{\partial x^{\prime \mu}}\right)=\frac{\partial x^{\alpha}}{\partial x^{\prime \mu}}(\varphi(\hat{x})) \hat{p}_{\alpha}-i \hbar\left(\frac{1}{2}-i \omega^{1}\right) \frac{\partial^{2} x^{\alpha}}{\partial x^{\prime \mu} \partial x^{\prime \nu}}(\varphi(\hat{x})) \frac{\partial x^{\prime \nu}}{\partial x^{\alpha}}(\hat{x}) .
$$

Assim, levando em conta (2.53), a equação (1.9) adquire a forma

$$
\hat{p}_{\mu}^{\prime}=\frac{\partial x^{\alpha}}{\partial x^{\prime \mu}}\left(\hat{x}^{\prime}\right) U \hat{p}_{\alpha} U^{\dagger}-i \hbar\left(\frac{1}{2}-i \omega^{1}\right) \frac{\partial^{2} x^{\alpha}}{\partial x^{\prime \mu} \partial x^{\prime \nu}}\left(\hat{x}^{\prime}\right) \frac{\partial x^{\prime \nu}}{\partial x^{\alpha}}\left(\varphi^{-1}\left(\hat{x}^{\prime}\right)\right) \text {. }
$$

Suponhamos agora que os vetores $|x\rangle \in \mathcal{R}$ obedeçam às condições (2.64), (2.65), necessárias para a definição de $\hat{D}(x, \xi, \omega)$ por $(2.66)$ e consideremos os vetores $\left|x^{\prime}\right\rangle^{\prime}=U|x\rangle \in \mathcal{R}^{\prime}$. A equação (2.53) e a unitariedade de $U$ garante que as equações (2.64) continuam valendo para $\left|x^{\prime}\right\rangle^{\prime}$, seguindo as mesmas demonstrações contidas 
nas equações (2.60) e (2.61). Para demonstrar a equação (2.65) notemos que

$$
\begin{aligned}
\left\langle\left. x^{\prime}\right|^{\prime} \frac{\partial x^{\alpha}}{\partial x^{\prime \mu}}\left(\hat{x}^{\prime}\right) U \hat{p}_{\alpha} U^{\dagger}\right. & =\frac{\partial x^{\alpha}}{\partial x^{\prime \mu}}\left(x^{\prime}\right)\left\langle\left. x^{\prime}\right|^{\prime} U \hat{p}_{\alpha} U^{\dagger}\right. \\
& =\frac{\partial x^{\alpha}}{\partial x^{\prime \mu}}\left(x^{\prime}\right)\langle x| \hat{p}_{\alpha} U^{\dagger} \\
& =-i \hbar \frac{\partial x^{\alpha}}{\partial x^{\prime \mu}}\left(x^{\prime}\right)\left(\frac{\partial}{\partial x^{\alpha}}+\left(\frac{1}{2}-i \omega^{1}\right) \Gamma_{\alpha}(x)\right)\langle x| U^{\dagger} \\
& =-i \hbar \frac{\partial x^{\alpha}}{\partial x^{\prime \mu}}\left(x^{\prime}\right)\left(\frac{\partial}{\partial x^{\alpha}}+\left(\frac{1}{2}-i \omega^{1}\right) \Gamma_{\alpha}(x)\right)\left\langle\left.\varphi(x)\right|^{\prime}\right. \\
& =-i \hbar\left(\frac{\partial}{\partial x^{\prime \mu}}+\left(\frac{1}{2}-i \omega^{1}\right) \frac{\partial x^{\alpha}}{\partial x^{\prime \mu}}\left(x^{\prime}\right) \Gamma_{\alpha}\left(\varphi^{-1}\left(x^{\prime}\right)\right)\right)\left\langle\left. x^{\prime}\right|^{\prime} .\right.
\end{aligned}
$$

Usando (3.4),

$$
\left\langle\left. x^{\prime}\right|^{\prime} \hat{p}_{\mu}^{\prime}=-i \hbar\left(\frac{\partial}{\partial x^{\prime \mu}}+\left(\frac{1}{2}-i \omega^{1}\right) \Gamma_{\mu}^{\prime}\left(x^{\prime}\right)\right)\left\langle\left. x^{\prime}\right|^{\prime}\right.\right.
$$

onde

$$
\Gamma_{\mu}^{\prime}\left(x^{\prime}\right)=\frac{\partial x^{\alpha}}{\partial x^{\prime \mu}}\left(x^{\prime}\right) \Gamma_{\alpha}\left(\varphi^{-1}\left(x^{\prime}\right)\right)+\frac{\partial^{2} x^{\alpha}}{\partial x^{\prime \mu} \partial x^{\prime \nu}}\left(x^{\prime}\right) \frac{\partial x^{\prime \nu}}{\partial x^{\alpha}}\left(\varphi^{-1}\left(x^{\prime}\right)\right)
$$

é a conexão de Levi-Civita contraída $\Gamma_{\rho \mu}^{\prime \rho}$ no sistema de coordenadas $x^{\prime}$, pela lei de transformação da conexão (ver apêndice B).

Assim, os vetores $\left|x^{\prime}\right\rangle^{\prime}=U|x\rangle \in \mathcal{R}^{\prime}$ obedecem às equações (2.64) e (2.65), que os definem a menos de uma fase global, e portanto definem o operador $\hat{D}^{\prime}\left(x^{\prime}, \xi^{\prime}, \omega\right)$ em $\mathcal{R}^{\prime}$ por

$$
\hat{D}^{\prime}\left(x^{\prime}, \xi^{\prime}, \omega\right)=\int\left|\gamma^{\prime}\left(x^{\prime},-\left(\frac{1}{2}+\theta\right) \xi^{\prime}\right)\right\rangle^{\prime}\left\langle\left.\gamma^{\prime}\left(x^{\prime},\left(\frac{1}{2}-\theta\right) \xi^{\prime}\right)\right|^{\prime} \omega(\theta) d \theta .\right.
$$

Pela propriedade (C.17) da função exponencial (ver apêndice C), temos $U|\gamma(x, \xi)\rangle=$ $|\varphi(\gamma(x, \xi))\rangle^{\prime}=\left|\gamma^{\prime}\left(\varphi(x), \frac{\partial x^{\prime}}{\partial x} \xi\right)\right\rangle^{\prime}$. Esta propriedade implica em

$$
U \hat{D}(x, \xi, \omega) U^{\dagger}=\hat{D}^{\prime}\left(\varphi(x), \frac{\partial x^{\prime}}{\partial x} \xi, \omega\right) .
$$


Pela definição (2.67),

$$
\begin{aligned}
U Q_{\omega, 1}(F) U^{\dagger} & =\int \tilde{F}(x, \xi) \hat{D}^{\prime}\left(\varphi(x), \frac{\partial x^{\prime}}{\partial x} \xi, \omega\right) \sqrt{g(x)} d x d \xi \\
& =\int \tilde{F}(x, \xi) \hat{D}^{\prime}\left(\varphi(x), \frac{\partial x^{\prime}}{\partial x} \xi, \omega\right) \sqrt{g(x)} d x d \xi \\
& =\int \widetilde{F^{\prime}}\left(x^{\prime}, \xi^{\prime}\right) \hat{D}^{\prime}\left(x^{\prime}, \xi^{\prime}, \omega\right) \sqrt{g^{\prime}\left(x^{\prime}\right)} d x^{\prime} d \xi^{\prime} \\
& =Q_{\omega, 1}^{\prime}\left(F^{\prime}\right),
\end{aligned}
$$

onde foi realizada na integral a mudança de variáveis $x^{\prime}=\varphi(x), \xi^{\prime}=\frac{\partial x^{\prime}}{\partial x} \xi$, levando em conta a lei de transformação da transformação de Fourier (2.43). Esta última equação demonstra que $Q_{\omega, 1}$ é covariante.

\subsection{Elemento de matriz de $Q_{\omega, 1}(F)$}

O elemento de matriz de $Q_{\omega, 1}(F)$, dado por (2.67), pode ser calculado considerando a normalização (2.64). Para tanto, vamos calcular o elemento de matriz de $\hat{D}(x, \xi, \omega)$, dado por (2.66):

$$
\left\langle\left. y\right|_{\omega^{1}} \hat{D}(x, \xi, \omega) \mid z\right\rangle_{\omega^{1}}=\int \frac{\delta^{D}\left(\gamma\left(x,-\left(\frac{1}{2}+\theta\right) \xi\right)-y\right)}{\sqrt{g(y)}} \frac{\delta^{D}\left(\gamma\left(x,\left(\frac{1}{2}-\theta\right) \xi\right)-z\right)}{\sqrt{g(z)}} \omega(\theta) d \theta .
$$

Nesta seção, deixaremos explícita a dimensionalidade da função delta. O produto das funções delta no integrando acima pode ser expresso como uma função delta de dimensão $2 D$ :

$\delta^{D}\left(\gamma\left(x,-\left(\frac{1}{2}+\theta\right) \xi\right)-y\right) \delta^{D}\left(\gamma\left(x,\left(\frac{1}{2}-\theta\right) \xi\right)-z\right)=\delta^{2 D}\left(\Phi_{\theta}(x, \xi)-(y, z)\right)$

onde $(y, z) \in \mathbb{R}^{2 D}$ e definimos a função $\Phi_{\theta}: \mathbb{R}^{2 D} \rightarrow \mathbb{R}^{2 D}$,

$$
\Phi_{\theta}(x, \xi)=\left(\gamma\left(x,-\left(\frac{1}{2}+\theta\right) \xi\right), \gamma\left(x,\left(\frac{1}{2}-\theta\right) \xi\right)\right) .
$$


Usando a lei de composição para a função exponencial (C.16), temos

$$
\begin{aligned}
\gamma\left(x,-\left(\frac{1}{2}+\theta\right) \xi\right) & =\gamma\left(x,\left(1+\frac{1}{2 \theta}\right)(-\theta \xi)\right) \\
& =\gamma\left(\gamma(x,-\theta \xi), \frac{1}{2 \theta} \beta(x,-\theta \xi)\right) \\
& =\gamma\left(\gamma(x,-\theta \xi), \frac{-1}{2}\left[\frac{1}{-\theta} \beta(x,-\theta \xi)\right]\right)
\end{aligned}
$$

e, da mesma maneira,

$$
\gamma\left(x,\left(\frac{1}{2}-\theta\right) \xi\right)=\gamma\left(\gamma(x,-\theta \xi), \frac{1}{2}\left[\frac{1}{-\theta} \beta(x,-\theta \xi)\right]\right) .
$$

Definindo a função $\Phi=\Phi_{0}$,

$$
\Phi(x, \xi)=\left(\gamma\left(x, \frac{-1}{2} \xi\right), \gamma\left(x, \frac{1}{2} \xi\right)\right)
$$

podemos expressar $\Phi_{\theta}$ através de $\Phi$ e do fluxo geodésico (C.13) como

$$
\Phi_{\theta}(x, \xi)=\Phi\left(T_{-\theta}(x, \xi)\right)
$$

o que permite representar o elemento de matriz de $\hat{D}(x, \xi, \omega)$ como

$$
\left\langle\left. y\right|_{\omega^{1}} \hat{D}(x, \xi, \omega) \mid z\right\rangle_{\omega^{1}}=\frac{1}{\sqrt{g(y) g(z)}} \int \delta^{2 D}\left(\Phi\left(T_{-\theta}(x, \xi)\right)-(y, z)\right) \omega(\theta) d \theta .
$$

Podemos simplificar a função delta na equação acima usando a propriedade

$$
\delta(f(x))=\sum_{k}\left|\operatorname{det}\left(\left.\frac{\partial f}{\partial x}\right|_{x=r_{k}}\right)\right|^{-1} \delta\left(x-r_{k}\right)
$$

onde $r_{k}$ são as raízes da equação $f(x)=0$. Precisamos, portanto, encontrar as raízes da equação $\Phi\left(T_{-\theta}(x, \xi)\right)=(y, z)$. Esta equação é equivalente ao sistema

$$
\begin{aligned}
\Phi(\bar{x}, \bar{\xi}) & =(y, z) \\
T_{-\theta}(x, \xi) & =(\bar{x}, \bar{\xi}) .
\end{aligned}
$$

A segunda equação tem solução imediata $(x, \xi)=T_{\theta}(\bar{x}, \bar{\xi})$, através da propriedade (C.15). As soluções da primeira equação estão em correspondência biunívoca com as geodésicas que unem o ponto $y$ ao ponto $z$. De fato, seja $K(\tau)$ uma geodésica, com $K(0)=y$ e $K(1)=z$. Então $\gamma(y, \zeta)=z$, com $\zeta=\dot{K}(0)$, e o tamanho do 


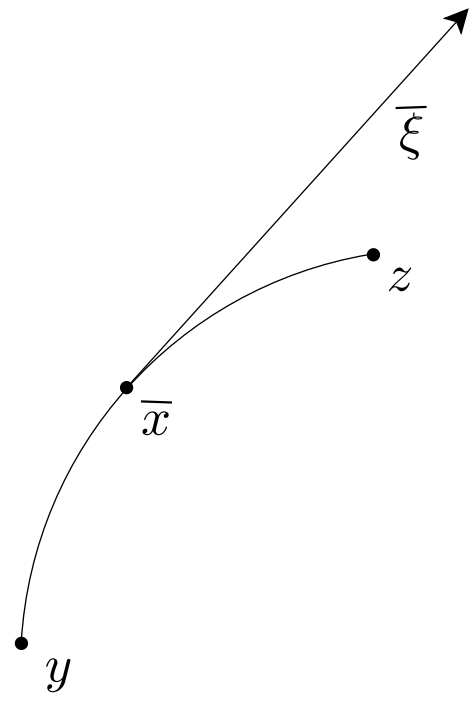

Figura 3.1: Ponto médio e vetor tangente à geodésica que une y a $z$.

vetor $\zeta$ é igual ao comprimento (C.2) da geodésica $K$, ou seja, $g_{\mu \nu}(y) \zeta^{\mu} \zeta^{\nu}=S[K]$. Definindo $\bar{x}=\gamma(y, \zeta / 2)$ e $\bar{\xi}=2 \beta(y, \zeta / 2)$, temos, pela lei de composição (C.16),

$$
\begin{aligned}
\gamma\left(\bar{x}, \frac{1}{2} \bar{\xi}\right) & =\gamma(\gamma(y, \zeta / 2), \beta(y, \zeta / 2))=\gamma(y, \zeta)=z \\
\gamma\left(\bar{x}, \frac{-1}{2} \bar{\xi}\right) & =\gamma(\gamma(y, \zeta / 2),-\beta(y, \zeta / 2))=\gamma(y, 0)=y
\end{aligned}
$$

ou seja, $\Phi(\bar{x}, \bar{\xi})=(y, z)$. $\bar{x}$ é o ponto médio da geodésica $K$, e $\bar{\xi}$ é um vetor tangente a $K$ no ponto $\bar{x}$, no sentido de $z$ (ver figura 3.1). Pela fórmula (C.7), seu comprimento é $g_{\mu \nu}(\bar{x}) \bar{\xi}^{\mu} \bar{\xi}^{\nu}=S[K]$. Por outro lado, se $\Phi(\bar{x}, \bar{\xi})=(y, z)$, definimos $\zeta=-2 \beta\left(\bar{x}, \frac{-1}{2} \bar{\xi}\right)$. A geodésica $K(\tau)=\gamma(y, \tau \zeta)$ é tal que $K(0)=\gamma(y, 0)=y$ e, usando a lei de composição (C.16),

$$
K(1)=\gamma\left(\gamma\left(\bar{x}, \frac{-1}{2} \bar{\xi}\right),-2 \beta\left(\bar{x}, \frac{-1}{2} \bar{\xi}\right)\right)=\gamma\left(\bar{x}, \frac{1}{2} \bar{\xi}\right)=z
$$

ou seja, a geodésica $K(\tau)$ une $y$ a $z$. Assim, se existem $n$ geodésicas $K_{(1)}, \ldots, K_{(n)}$ que unem $y$ a $z$, com pontos médios $\bar{x}_{(1)}, \ldots, \bar{x}_{(n)}$ e vetores tangentes $\bar{\xi}_{(1)}, \ldots, \bar{\xi}_{(n)}$, existirão $n$ soluções $T_{\theta}\left(\bar{x}_{(1)}, \bar{\xi}_{(1)}\right), \ldots, T_{\theta}\left(\bar{x}_{(n)}, \bar{\xi}_{(n)}\right)$ da equação $\Phi\left(T_{-\theta}(x, \xi)\right)=(y, z)$.

Em seguida, precisamos calcular o determinante jacobiano da função composta 
$\Phi\left(T_{-\theta}(x, \xi)\right)-(y, z) .(y, z)$ é uma constante, logo o determinante procurado é

$$
\begin{aligned}
\operatorname{det}\left(\frac{\partial \Phi\left(T_{-\theta}(x, \xi)\right)}{\partial(x, \xi)}\right) & =\operatorname{det}\left(\frac{\partial \Phi}{\partial(x, \xi)}\left(T_{-\theta}(x, \xi)\right)\right) \operatorname{det}\left(\frac{\partial T_{-\theta}}{\partial(x, \xi)}\right) \\
& =\operatorname{det}\left(\frac{\partial \Phi}{\partial(x, \xi)}\left(T_{-\theta}(x, \xi)\right)\right) \frac{g(x)}{g(\gamma(x,-\theta \xi))}
\end{aligned}
$$

onde foi usado o resultado da seção C.3 para o determinante jacobiano do fluxo geodésico. Assim, levando em conta que $T_{-\theta}\left(T_{\theta}\left(\bar{x}_{(n)}, \bar{\xi}_{(n)}\right)\right)=\left(\bar{x}_{(n)}, \bar{\xi}_{(n)}\right)$ e que

$$
\left.\frac{g(x)}{g(\gamma(x,-\theta \xi))}\right|_{(x, \xi)=T_{\theta}\left(\bar{x}_{(k)}, \bar{\xi}_{(k)}\right)}=\frac{g\left(\gamma\left(\bar{x}_{(k)}, \theta \bar{\xi}_{(k)}\right)\right)}{g\left(\bar{x}_{(k)}\right)}
$$

temos

$$
\frac{\delta^{2 D}\left(\Phi\left(T_{-\theta}(x, \xi)\right)-(y, z)\right)}{\sqrt{g(y) g(z)}}=\sum_{k=1}^{n} \frac{\delta^{2 D}\left((x, \xi)-T_{\theta}\left(\bar{x}_{(k)}, \bar{\xi}_{(k)}\right)\right)}{\mathcal{M}\left(\bar{x}_{(k)}, \bar{\xi}_{(k)}\right) g\left(\gamma\left(\bar{x}_{(k)}, \theta \bar{\xi}_{(k)}\right)\right)}
$$

onde definimos a função

$$
\mathcal{M}(x, \xi)=\frac{\sqrt{g\left(\gamma\left(x, \frac{-1}{2} \xi\right)\right) g\left(\gamma\left(x, \frac{1}{2} \xi\right)\right)}}{g(x)}\left|\operatorname{det}\left(\frac{\partial \Phi}{\partial(x, \xi)}(x, \xi)\right)\right|
$$

(note-se que $g(y)=g\left(\gamma\left(\bar{x}_{(k)}, \frac{-1}{2} \bar{\xi}_{(k)}\right)\right)$ e $g(z)=g\left(\gamma\left(\bar{x}_{(k)}, \frac{1}{2} \bar{\xi}_{(k)}\right)\right)$ para todo $\left.k\right)$. Juntando (3.6) e (3.7), obtemos

$$
\left\langle\left. y\right|_{\omega^{1}} \hat{D}(x, \xi, \omega) \mid z\right\rangle_{\omega^{1}}=\sum_{k=1}^{n} \frac{1}{\mathcal{M}\left(\bar{x}_{(k)}, \bar{\xi}_{(k)}\right)} \int \frac{\delta^{2 D}\left((x, \xi)-T_{\theta}\left(\bar{x}_{(k)}, \bar{\xi}_{(k)}\right)\right)}{g\left(\gamma\left(\bar{x}_{(k)}, \theta \bar{\xi}_{(k)}\right)\right)} \omega(\theta) d \theta .
$$

Pela definição (2.67), podemos calcular o elemento de matriz de $Q_{\omega, 1}(F)$ como

$$
\begin{aligned}
\left\langle\left. y\right|_{\omega^{1}} Q_{\omega, 1}(F) \mid z\right\rangle_{\omega^{1}}= & \int \tilde{F}(x, \xi)\left\langle\left. y\right|_{\omega^{1}} \hat{D}(x, \xi, \omega) \mid z\right\rangle_{\omega^{1}} \sqrt{g(x)} d x d \xi \\
= & \sum_{k=1}^{n} \frac{1}{\mathcal{M}\left(\bar{x}_{(k)}, \bar{\xi}_{(k)}\right)} \times \\
& \times \int \tilde{F}(x, \xi) \frac{\delta^{2 D}\left((x, \xi)-T_{\theta}\left(\bar{x}_{(k)}, \bar{\xi}_{(k)}\right)\right)}{g\left(\gamma\left(\bar{x}_{(k)}, \theta \bar{\xi}_{(k)}\right)\right)} \sqrt{g(x)} d x d \xi \omega(\theta) d \theta \\
= & \sum_{k=1}^{n} \frac{1}{\mathcal{M}\left(\bar{x}_{(k)}, \bar{\xi}_{(k)}\right)} \int \frac{\tilde{F}\left(T_{\theta}\left(\bar{x}_{(k)}, \bar{\xi}_{(k)}\right)\right)}{\sqrt{g\left(\gamma\left(\bar{x}_{(k)}, \theta \bar{\xi}_{(k)}\right)\right)}} \omega(\theta) d \theta
\end{aligned}
$$


onde usamos o fato de que

$$
\left.\sqrt{g(x)}\right|_{(x, \xi)=T_{\hbar \theta}\left(\bar{x}_{(k)}, \bar{\xi}_{(k)}\right)}=\sqrt{g\left(\gamma\left(\bar{x}_{(k)}, \hbar \theta \bar{\xi}_{(k)}\right)\right)} .
$$

A expressão (3.9), válida para qualquer função $F$, pode ser bastante simplificada no caso particular de $F(x, p)$ polinomial em $p$. Neste caso,

$$
\tilde{F}(x, \xi)=F\left(x, i \hbar \frac{\partial}{\partial \xi}\right) \delta(\xi)
$$

é uma distribuição cujo suporte é $\xi=0$. Assim,

$$
\tilde{F}\left(T_{\theta}\left(\bar{x}_{(k)}, \bar{\xi}_{(k)}\right)\right)=\tilde{F}\left(\gamma\left(\bar{x}_{(k)}, \theta \bar{\xi}_{(k)}\right), \frac{1}{\theta} \beta\left(\bar{x}_{(k)}, \theta \bar{\xi}_{(k)}\right)\right)
$$

será sempre zero, a não ser que a equação

$$
\frac{1}{\theta} \beta\left(\bar{x}_{(k)}, \theta \bar{\xi}_{(k)}\right)=0 \Leftrightarrow \bar{\xi}_{(k)}=0
$$

seja possível. De todas as geodésicas $K_{(k)}$ que unem $y$ a $z$, apenas a geodésica mínima $C_{y z}$ satisfaz esta propriedade (ver a seção C.2), quando $y \rightarrow z$. Assim, se $F$ é polinomial, a soma em (3.9) se reduz a um único termo correspondente à geodésica mínima como

$$
\left\langle\left. y\right|_{\omega^{1}} Q_{\omega, 1}(F) \mid z\right\rangle_{\omega^{1}}=\frac{1}{\mathcal{M}(\bar{x}, \bar{\xi})} \int \frac{\tilde{F}\left(T_{\theta}(\bar{x}, \bar{\xi})\right)}{\sqrt{g(\gamma(\bar{x}, \theta \bar{\xi}))}} \omega(\theta) d \theta,
$$

onde $\bar{x}=\bar{x}(y, z)$ e $\bar{\xi}=\bar{\xi}(y, z)$ são o ponto médio e o vetor tangente da geodésica mínima $C_{y z}$. Vamos tratar apenas de funções polinomiais em $p$. Levando em conta que a transformação de Fourier $F \rightarrow \tilde{F}$ atua apenas na variável $p$, a equação (3.10) pode ser escrita como

$$
\left\langle\left. y\right|_{\omega^{1}} Q_{\omega, 1}(F) \mid z\right\rangle_{\omega^{1}}=\frac{1}{\mathcal{M}(\bar{x}, \bar{\xi})} \int \widetilde{\left(\frac{F}{\sqrt{g}}\right)}\left(T_{\theta}(\bar{x}, \bar{\xi})\right) \omega(\theta) d \theta
$$

O elemento de matriz da primeira generalização da quantização de Weyl $Q^{\mathrm{W}, 1}$ é obtido substituindo $\omega(\theta)=\delta(\theta)$ na fórmula acima. Para esta quantização,

$$
\omega^{1}=i \int \theta \delta(\theta) d \theta=0
$$

Levando em conta que o fluxo geodésico obedece à propriedade $T_{0}(\bar{x}, \bar{\xi})=(\bar{x}, \bar{\xi})$, 
temos

$$
\begin{aligned}
\left\langle\left. y\right|_{0} Q^{\mathrm{W}, 1}(F) \mid z\right\rangle_{0} & =\frac{1}{\mathcal{M}(\bar{x}, \bar{\xi})} \widetilde{\left(\frac{F}{\sqrt{g}}\right)}(\bar{x}, \bar{\xi}) \\
& =\frac{\tilde{F}(\bar{x}, \bar{\xi})}{\mathcal{M}(\bar{x}, \bar{\xi}) \sqrt{g(\bar{x})}} .
\end{aligned}
$$

\section{$3.4 \omega, 1$-símbolos}

Como na seção (2.4), dado um operador $\hat{F}$, se $\hat{F}=Q_{\omega, 1}(F)$, dizemos que a função $F(x, p)$ é o símbolo do operador $\hat{F}$ segundo a quantização $Q_{\omega, 1}$, ou o $\omega, 1$-símbolo de $\hat{F}$, que será representado por $F_{\omega, 1}(x, p)$. As fórmulas (3.11) e (3.12) relacionan o elemento de matriz de um operador $\hat{F}$ com seu $\omega$, 1-símbolo e seu primeiro símbolo de Weyl como

$$
\begin{aligned}
\left\langle\left. y\right|_{\omega^{1}} \hat{F} \mid z\right\rangle_{\omega^{1}} & =\frac{1}{\mathcal{M}(\bar{x}, \bar{\xi})} \int \widetilde{\left(\frac{F_{\omega, 1}}{\sqrt{g}}\right)}\left(T_{\theta}(\bar{x}, \bar{\xi})\right) \omega(\theta) d \theta \\
\left\langle\left. y\right|_{0} \hat{F} \mid z\right\rangle_{0} & =\frac{\widetilde{F^{\mathrm{W}, 1}}(\bar{x}, \bar{\xi})}{\mathcal{M}(\bar{x}, \bar{\xi}) \sqrt{g(\bar{x})}},
\end{aligned}
$$

onde $\bar{x}=\bar{x}(y, z)$ e $\bar{\xi}=\bar{\xi}(y, z)$ são o ponto médio e o vetor tangente da geodésica mínima que une $y$ a $z$. Pela equação (2.69), temos

$$
\left\langle\left. y\right|_{\omega^{1}} \hat{F} \mid z\right\rangle_{\omega^{1}}=e^{i \omega^{1}(\ln \sqrt{g(y)}-\ln \sqrt{g(z)})}\left\langle\left. y\right|_{0} \hat{F} \mid z\right\rangle_{0} .
$$

Levando em conta que $y=\gamma\left(\bar{x}, \frac{-1}{2} \bar{\xi}\right)$ e $z=\gamma\left(\bar{x}, \frac{1}{2} \bar{\xi}\right)$, temos a relação entre o $\omega, 1$-símbolo e o primeiro símbolo de Weyl

$$
\widetilde{F^{\mathrm{W}, 1}}(x, \xi)=\Lambda_{\omega^{1}}(x, \xi) \sqrt{g(x)} \int \widetilde{\left(\frac{F_{\omega, 1}}{\sqrt{g}}\right)}\left(T_{\theta}(x, \xi)\right) \omega(\theta) d \theta
$$

onde definimos a função

$$
\begin{aligned}
& \Lambda_{\omega^{1}}(x, \xi)=e^{i \omega^{1}\left(\ln \sqrt{g\left(\gamma\left(x, \frac{1}{2} \xi\right)\right)}-\ln \sqrt{g\left(\gamma\left(x, \frac{-1}{2} \xi\right)\right)}\right)} \\
& =\exp \left(i \omega^{1} \xi^{\mu} \Gamma_{\mu}(x)+O\left(\xi^{3}\right)\right) \\
& =1+i \omega^{1} \xi^{\mu} \Gamma_{\mu}(x)-\left(\omega^{1} \xi^{\mu} \Gamma_{\mu}(x)\right)^{2}+O\left(\xi^{3}\right) .
\end{aligned}
$$

A expansão de $\ln \sqrt{g\left(\gamma\left(x, \pm \frac{1}{2} \xi\right)\right)}$ decorre de (C.25).

Esta expressão pode ser colocada na forma de uma série de potências de $\hbar$. Para 
tanto, precisaremos do resultado a seguir. Consideremos uma função $\tilde{A}(x, \xi)$ que é a transformação de Fourier em relação a $p$ de $A(x, p)$ dada por (2.41). Pelas fórmulas (C.11) e (C.12), podemos calcular a derivada

$$
\begin{aligned}
\frac{d}{d \theta} \tilde{A}\left(T_{\theta}(x, \xi)\right) & =\frac{d}{d \theta} \tilde{A}\left(x_{\theta}, \xi_{\theta}\right) \\
& =\frac{\partial \tilde{A}}{\partial x^{\mu}}\left(x_{\theta}, \xi_{\theta}\right) \xi_{\theta}^{\mu}-\frac{\partial \tilde{A}}{\partial \xi^{\mu}}\left(x_{\theta}, \xi_{\theta}\right) \Gamma_{\alpha \beta}^{\mu}\left(x_{\theta}\right) \xi_{\theta}^{\alpha} \xi_{\theta}^{\beta}
\end{aligned}
$$

A multiplicação de $\tilde{A}$ por $\xi^{\mu}$ corresponde à derivação - $i \hbar \frac{\partial}{\partial p_{\mu}}$ de $A$ pela transformação (2.41). Desta maneira, o primeiro termo pode ser escrito como

$$
\begin{aligned}
\frac{\partial \tilde{A}}{\partial x^{\mu}}\left(x_{\theta}, \xi_{\theta}\right) \xi_{\theta}^{\mu} & =\frac{\widetilde{\partial A}}{\frac{\partial x^{\mu}}{\partial^{2} A}}\left(x_{\theta}, \xi_{\theta}\right) \xi_{\theta}^{\mu} \\
& =-i \hbar \frac{\left.\partial_{\theta}, \xi_{\theta}\right)}{\partial x^{\mu} \partial p_{\mu}}
\end{aligned}
$$

Analogamente, a derivação $\frac{\partial}{\partial \xi^{\mu}}$ de $\tilde{A}$ corresponde à multiplicação de $A$ por $-\frac{i}{\hbar} p_{\mu}$. Portanto, o segundo termo pode ser escrito como

$$
\begin{aligned}
\frac{\partial \tilde{A}}{\partial \xi^{\mu}}\left(x_{\theta}, \xi_{\theta}\right) \Gamma_{\alpha \beta}^{\mu}\left(x_{\theta}\right) \xi_{\theta}^{\alpha} \xi_{\theta}^{\beta} & =-\frac{i}{\hbar} \widehat{\left(p_{\mu} A\right)}\left(x_{\theta}, \xi_{\theta}\right) \Gamma_{\alpha \beta}^{\mu}\left(x_{\theta}\right) \xi_{\theta}^{\alpha} \xi_{\theta}^{\beta} \\
& =-\frac{i}{\hbar}\left(\Gamma_{\alpha \beta}^{\mu} p_{\mu} A\right)^{\sim}\left(x_{\theta}, \xi_{\theta}\right) \xi_{\theta}^{\alpha} \xi_{\theta}^{\beta} \\
& =i \hbar\left(\Gamma_{\alpha \beta}^{\mu} \frac{\partial^{2}}{\partial p_{\alpha} \partial p_{\beta}}\left(p_{\mu} A\right)\right)^{\sim}\left(x_{\theta}, \xi_{\theta}\right)
\end{aligned}
$$

Assim, a derivada (3.15) pode ser escrita como

$$
\frac{d}{d \theta} \tilde{A}\left(T_{\theta}(x, \xi)\right)=\widetilde{-i \hbar \Delta^{\prime}} A\left(T_{\theta}(x, \xi)\right)
$$

onde definimos o operador $\Delta^{\prime}$ que atua sobre as funções clássicas como

$$
\begin{aligned}
\Delta^{\prime} A(x, p) & =\frac{\partial^{2} A}{\partial x^{\mu} \partial p_{\mu}}(x, p)+\Gamma_{\alpha \beta}^{\mu}(x) \frac{\partial^{2}}{\partial p_{\alpha} \partial p_{\beta}}\left(p_{\mu} A(x, p)\right) \\
& =\left(\frac{\partial^{2}}{\partial x^{\mu} \partial p_{\mu}}+2 \Gamma_{\alpha}(x) \frac{\partial}{\partial p_{\alpha}}+p_{\mu} \Gamma_{\alpha \beta}^{\mu}(x) \frac{\partial^{2}}{\partial p_{\alpha} \partial p_{\beta}}\right) A(x, p)
\end{aligned}
$$

Naturalmente, como o segundo membro de (3.16) ainda é uma função de $T_{\theta}(x, \xi)$, 
valem as fórmulas

$$
\begin{aligned}
\frac{d^{n}}{d \theta^{n}} \tilde{A}\left(T_{\theta}(x, \xi)\right) & =\left(\left(-i \hbar \Delta^{\prime}\right)^{n} A\right)^{\sim}\left(T_{\theta}(x, \xi)\right) \\
\left.\frac{d^{n}}{d \theta^{n}} \tilde{A}\left(T_{\theta}(x, \xi)\right)\right|_{\theta=0} & =\left(\left(-i \hbar \Delta^{\prime}\right)^{n} A\right)^{\sim}(x, \xi) .
\end{aligned}
$$

Finalmente, podemos expressar $\tilde{A}\left(T_{\theta}(x, \xi)\right)$ como uma série de Taylor

$$
\tilde{A}\left(T_{\theta}(x, \xi)\right)=\left(\sum_{n=0}^{\infty} \frac{\theta^{n}}{n !}\left(-i \hbar \Delta^{\prime}\right)^{n} A\right)^{\sim}(x, \xi) .
$$

Para expressar (3.13), precisamos saber como o operador $\Delta^{\prime}$ opera sobre $A(x, p)=$ $F(x, p) / \sqrt{g(x)}$. Levando em conta a fórmula (B.2) e que

$$
\frac{\partial}{\partial x^{\mu}}\left(\frac{1}{\sqrt{g(x)}}\right)=\frac{1}{\sqrt{g(x)}} \frac{\partial}{\partial x^{\mu}} \ln \left(\frac{1}{\sqrt{g(x)}}\right)=-\frac{1}{\sqrt{g(x)}} \Gamma_{\mu}(x),
$$

e, portanto

$$
\begin{aligned}
\Delta^{\prime}\left(\frac{F(x, p)}{\sqrt{g(x)}}\right)= & \left(\frac{\partial}{\partial x^{\mu}} \frac{1}{\sqrt{g(x)}} \frac{\partial}{\partial p_{\mu}}+\frac{1}{\sqrt{g(x)}} 2 \Gamma_{\alpha}(x) \frac{\partial}{\partial p_{\alpha}}\right. \\
& \left.\quad+\frac{1}{\sqrt{g(x)}} p_{\mu} \Gamma_{\alpha \beta}^{\mu}(x) \frac{\partial^{2}}{\partial p_{\alpha} \partial p_{\beta}}\right) F(x, p) \\
= & \frac{1}{\sqrt{g(x)}} \Delta F(x, p) \\
\left(\Delta^{\prime}\right)^{n}\left(\frac{F(x, p)}{\sqrt{g(x)}}\right)= & \frac{1}{\sqrt{g(x)}} \Delta^{n} F(x, p)
\end{aligned}
$$

onde definimos o operador

$$
\Delta=\frac{\partial^{2}}{\partial x^{\mu} \partial p_{\mu}}+\Gamma_{\alpha}(x) \frac{\partial}{\partial p_{\alpha}}+p_{\mu} \Gamma_{\alpha \beta}^{\mu}(x) \frac{\partial^{2}}{\partial p_{\alpha} \partial p_{\beta}} .
$$

Temos assim, usando a fórmula (3.17), a expansão

$$
\widetilde{\left(\frac{F}{\sqrt{g}}\right)}\left(T_{\theta}(x, \xi)\right)=\frac{1}{\sqrt{g(x)}}\left(\sum_{k=0}^{\infty} \frac{(i \theta)^{n}}{n !}(-\hbar \Delta)^{n} F\right)^{\sim}(x, \xi),
$$


e, portanto, a desejada expansão em potências de $\hbar$ de $(3.13)$

$$
\begin{aligned}
\widetilde{F^{\mathrm{W}, 1}}(x, \xi) & =\Lambda_{\omega^{1}}(x, \xi)\left(\sum_{k=0}^{\infty} \frac{\omega^{n}}{n !}(-\hbar \Delta)^{n} F_{\omega, 1}\right)^{\sim}(x, \xi) \\
& =\left(\Lambda_{\omega^{1}}\left(x,-i \hbar \frac{\partial}{\partial p}\right) \sum_{k=0}^{\infty} \frac{\omega^{n}}{n !}(-\hbar \Delta)^{n} F_{\omega, 1}\right)^{\sim}(x, \xi),
\end{aligned}
$$

onde definimos o $k$-momento da distribuição $\omega(\theta)$

$$
\omega^{n}=\int(i \theta)^{n} \omega(\theta) d \theta
$$

A transformação de Fourier (2.41) é inversível. Logo, a equação (3.19) implica

$$
F^{\mathrm{W}, 1}(x, p)=\Lambda_{\omega^{1}}\left(x,-i \hbar \frac{\partial}{\partial p}\right) \sum_{n=0}^{\infty} \frac{\omega^{n}}{n !}(-\hbar \Delta)^{n} F_{\omega, 1}(x, p) .
$$

Note-se que o expoente de (3.14) é uma função ímpar, logo a extensão analítica de $\Lambda_{\omega^{1}}$ para o argumento $\xi$ imaginário $\Lambda_{\omega^{1}}\left(x,-i \hbar \frac{\partial}{\partial p}\right)$ é real. Definindo a função $\Omega(k)$ pela transformação de Fourier (2.25), vemos que

$$
\frac{d^{n} \Omega}{d k^{n}}(0)=\omega^{n}
$$

Assim, a equação (3.20) pode ser escrita como

$$
F^{\mathrm{W}, 1}(x, p)=\Lambda_{\omega^{1}}\left(x,-i \hbar \frac{\partial}{\partial p}\right) \Omega(-\hbar \Delta) F_{\omega, 1}(x, p)
$$

A condição de normalização $\omega^{0}=1$ implica em $\Omega(0)=1$, como no caso do espaço plano. O operador $\Delta$ é a generalização a espaços curvos ou planos em coordenadas gerais do operador (2.39). De fato, se $x$ são coordenadas retilíneas em um espaço 
plano e $x^{\prime}=\varphi(x)$ são coordenadas gerais, e $F(x, p)=F^{\prime}\left(x^{\prime}, p^{\prime}\right)=F^{\prime}\left(\varphi(x), \frac{\partial x}{\partial x^{\prime}} p\right)$,

$$
\begin{aligned}
\frac{\partial^{2}}{\partial x^{\mu} \partial p_{\mu}} F(x, p)= & \frac{\partial}{\partial x^{\mu}} \frac{\partial}{\partial p_{\mu}} F^{\prime}\left(\varphi(x), \frac{\partial x}{\partial x^{\prime}} p\right)=\frac{\partial}{\partial x^{\mu}}\left(\frac{\partial F^{\prime}}{\partial p_{\alpha}^{\prime}}\left(\varphi(x), \frac{\partial x}{\partial x^{\prime}} p\right) \frac{\partial x^{\mu}}{\partial x^{\prime \alpha}}\right) \\
= & \frac{\partial^{2} F^{\prime}}{\partial x^{\prime \beta} \partial p_{\alpha}^{\prime}}\left(\varphi(x), \frac{\partial x}{\partial x^{\prime}} p\right) \frac{\partial x^{\prime \beta}}{\partial x^{\mu}} \frac{\partial x^{\mu}}{\partial x^{\prime \alpha}} \\
& \quad+\frac{\partial^{2} F^{\prime}}{\partial p_{\beta}^{\prime} \partial p_{\alpha}^{\prime}}\left(\varphi(x), \frac{\partial x}{\partial x^{\prime}} p\right) \frac{\partial x^{\mu}}{\partial x^{\prime \alpha}} \frac{\partial}{\partial x^{\mu}}\left(\frac{\partial x^{\rho}}{\partial x^{\prime \beta}}\right) p_{\rho} \\
& +\frac{\partial F^{\prime}}{\partial p_{\alpha}^{\prime}}\left(\varphi(x), \frac{\partial x}{\partial x^{\prime}} p\right) \frac{\partial}{\partial x^{\mu}}\left(\frac{\partial x^{\mu}}{\partial x^{\prime \alpha}}\right) \\
= & \frac{\partial^{2} F^{\prime}}{\partial x^{\prime \mu} \partial p_{\mu}^{\prime}}+\frac{\partial^{2} F^{\prime}}{\partial p_{\beta}^{\prime} \partial p_{\alpha}^{\prime}} \frac{\partial^{2} x^{\rho}}{\partial x^{\prime \alpha} \partial x^{\prime \beta}} \frac{\partial x^{\prime \kappa}}{\partial x^{\rho}} p_{\kappa}^{\prime}+\frac{\partial F^{\prime}}{\partial p_{\alpha}^{\prime}} \frac{\partial^{2} x^{\mu}}{\partial x^{\prime \rho} \partial x^{\prime \alpha}} \frac{\partial x^{\prime \rho}}{\partial x^{\mu}} \\
= & \frac{\partial^{2} F^{\prime}}{\partial x^{\prime \mu} \partial p_{\mu}^{\prime}}+\frac{\partial^{2} F^{\prime}}{\partial p_{\beta}^{\prime} \partial p_{\alpha}^{\prime}} \Gamma_{\alpha \beta}^{\prime \kappa}\left(x^{\prime}\right) p_{\kappa}^{\prime}+\frac{\partial F^{\prime}}{\partial p_{\alpha}^{\prime}} \Gamma_{\alpha}^{\prime}\left(x^{\prime}\right) .
\end{aligned}
$$

\subsection{Propriedades da função $\mathcal{M}(x, \xi)$}

Definindo a função

$$
\gamma_{a}^{\mu}(x, \xi)=\gamma^{\mu}(x, a \xi)
$$

podemos expressar a função definida por (3.8) e (3.5) como

$$
\begin{aligned}
\mathcal{M}(x, \xi) & =\frac{\sqrt{g\left(\gamma_{-1 / 2}(x, \xi)\right) g\left(\gamma_{1 / 2}(x, \xi)\right)}}{g(x)} f(x, \xi), \\
f(x, \xi) & =\left|\operatorname{det}\left(\frac{\partial \Phi}{\partial(x, \xi)}(x, \xi)\right)\right|=\left|\operatorname{det}\left(\begin{array}{cc}
\frac{\partial \gamma_{-1 / 2}}{\partial x} & \frac{\partial \gamma_{-1 / 2}}{\partial \xi} \\
\frac{\partial \gamma_{1 / 2}}{\partial x} & \frac{\partial \gamma_{1 / 2}}{\partial \xi}
\end{array}\right)\right|,
\end{aligned}
$$

onde as submatrizes $D \times D$ são

$$
\begin{gathered}
\frac{\partial \gamma_{a}}{\partial x}=\left(\frac{\partial \gamma_{a}^{\mu}}{\partial x^{\nu}}\right)=\left(\begin{array}{cccc}
\frac{\partial \gamma_{a}^{1}}{\partial x^{1}} & \frac{\partial \gamma_{a}^{1}}{\partial x^{2}} & \cdots & \frac{\partial \gamma_{a}^{1}}{\partial x^{D}} \\
\frac{\partial \gamma_{a}^{2}}{\partial x^{1}} & \frac{\partial \gamma_{a}^{2}}{\partial x^{2}} & \cdots & \frac{\partial \gamma_{a}^{2}}{\partial x^{D}} \\
\vdots & & & \\
\frac{\partial \gamma_{a}^{D}}{\partial x^{1}} & \frac{\partial \gamma_{a}^{D}}{\partial x^{2}} & \cdots & \frac{\partial \gamma_{a}^{D}}{\partial x^{D}}
\end{array}\right) \\
\frac{\partial \gamma_{a}}{\partial \xi}=\left(\frac{\partial \gamma_{a}^{\mu}}{\partial \xi^{\nu}}\right)=\left(\begin{array}{cccc}
\frac{\partial \gamma_{a}^{1}}{\partial \xi^{1}} & \frac{\partial \gamma_{a}^{1}}{\partial \xi^{2}} & \cdots & \frac{\partial \gamma_{a}^{1}}{\partial \xi^{D}} \\
\frac{\partial \gamma_{a}^{2}}{\partial \xi^{1}} & \frac{\partial \gamma_{a}^{2}}{\partial \xi^{2}} & \cdots & \frac{\partial \gamma_{a}^{2}}{\partial \xi^{D}} \\
\vdots & & & \\
\frac{\partial \gamma_{a}^{D}}{\partial \xi^{1}} & \frac{\partial \gamma_{a}^{D}}{\partial \xi^{2}} & \cdots & \frac{\partial \gamma_{a}^{D}}{\partial \xi^{D}}
\end{array}\right) .
\end{gathered}
$$


A funções $\sqrt{g\left(\gamma_{-1 / 2}(x, \xi)\right) g\left(\gamma_{1 / 2}(x, \xi)\right)}$ e $f(x, \xi)$ são claramente pares em $\xi$. Assim, por (3.23), deduzimos a propriedade de que $\mathcal{M}(x, \xi)$ é par em $\xi$ :

$$
\mathcal{M}(x, \xi)=\mathcal{M}(x,-\xi)
$$

Em um novo sistema de coordenadas

$$
x^{\prime \mu}=\varphi(x), \xi^{\prime \mu}=\frac{\partial x^{\prime \mu}}{\partial x^{\nu}} \xi^{\nu}=\frac{\partial \varphi^{\mu}}{\partial x^{\nu}}(x) \xi^{\nu}
$$

$\mathcal{M}^{\prime}$ está definida como

$$
\begin{aligned}
\mathcal{M}^{\prime}\left(x^{\prime}, \xi^{\prime}\right) & =\frac{\sqrt{g^{\prime}\left(\gamma_{-1 / 2}^{\prime}\right) g\left(\gamma_{1 / 2}^{\prime}\right)}}{g(x)} f^{\prime}\left(x^{\prime}, \xi^{\prime}\right) \\
f^{\prime}\left(x^{\prime}, \xi^{\prime}\right) & =\left|\operatorname{det}\left(\begin{array}{cc}
\frac{\partial \gamma_{-1 / 2}^{\prime}}{\partial x^{\prime}} & \frac{\partial \gamma_{-1 / 2}^{\prime}}{\partial \xi^{\prime}} \\
\frac{\partial \gamma_{1 / 2}^{\prime}}{\partial x^{\prime}} & \frac{\partial \gamma_{1 / 2}^{\prime}}{\partial \xi^{\prime}}
\end{array}\right)\right|,
\end{aligned}
$$

onde $\gamma_{a}^{\prime}\left(x^{\prime}, \xi^{\prime}\right)=\gamma^{\prime}\left(x^{\prime}, a \xi^{\prime}\right)$. Pela lei de transformação (C.17)

$$
\gamma_{a}^{\prime \mu}\left(x^{\prime}, \xi^{\prime}\right)=\gamma^{\prime \mu}\left(x^{\prime}, a \xi^{\prime}\right)=\varphi^{\mu}(\gamma(x, a \xi))=\varphi^{\mu}\left(\gamma_{a}(x, \xi)\right)
$$

onde $x^{\prime}$ e $\xi^{\prime}$ se relacionam por $(3.24)$ (o que implica que $\left(a \xi^{\prime}\right)^{\mu}=\frac{\partial x^{\prime \mu}}{\partial x^{\nu}}(a \xi)^{\nu}=$ $\left.\frac{\partial \varphi^{\mu}}{\partial x^{\nu}}(x)(a \xi)^{\nu}\right)$, temos

$$
\begin{aligned}
& \frac{\partial \gamma_{a}^{\prime \mu}}{\partial x^{\prime \nu}}=\frac{\partial \varphi^{\mu}}{\partial x^{\alpha}}\left(\gamma_{a}\right)\left(\frac{\partial \gamma_{a}^{\alpha}}{\partial x^{\beta}} \frac{\partial x^{\beta}}{\partial x^{\prime \nu}}+\frac{\partial \gamma_{a}^{\alpha}}{\partial \xi^{\beta}} \frac{\partial \xi^{\beta}}{\partial x^{\prime \nu}}\right) \\
& \frac{\partial \gamma_{a}^{\prime \mu}}{\partial \xi^{\prime \nu}}=\frac{\partial \varphi^{\mu}}{\partial x^{\alpha}}\left(\gamma_{a}\right) \frac{\partial \gamma_{a}^{\alpha}}{\partial \xi^{\beta}} \frac{\partial \xi^{\beta}}{\partial \xi^{\prime \nu}}=\frac{\partial \varphi^{\mu}}{\partial x^{\alpha}}\left(\gamma_{a}\right) \frac{\partial \gamma_{a}^{\alpha}}{\partial \xi^{\beta}} \frac{\partial x^{\beta}}{\partial x^{\prime \nu}}
\end{aligned}
$$

onde usamos a transformação inversa $x^{\mu}=\varphi^{-1 \mu}\left(x^{\prime}\right), \xi^{\mu}=\frac{\partial x^{\mu}}{\partial x^{\prime \nu}} \xi^{\prime \nu}$. Definindo as matrizes

$$
A_{a \nu}^{\mu}=\frac{\partial \varphi^{\mu}}{\partial x^{\nu}}\left(\gamma_{a}\right), B_{\nu}^{\mu}=\frac{\partial x^{\mu}}{\partial x^{\prime \nu}}, C_{\nu}^{\mu}=\frac{\partial \xi^{\mu}}{\partial x^{\prime \nu}}
$$

expressamos $f^{\prime}$ (3.26) como o módulo do determinante da matriz

$$
\begin{aligned}
& \left(\begin{array}{cc}
A_{\frac{-1}{2}} \frac{\partial \gamma_{-1 / 2}}{\partial x} B+A_{\frac{-1}{2}} \frac{\partial \gamma_{-1 / 2}}{\partial \xi} C & A_{\frac{-1}{2}} \frac{\partial \gamma_{-1 / 2}}{\partial \xi} B \\
A_{\frac{1}{2}} \frac{\partial \gamma_{1 / 2}}{\partial x} B+A_{\frac{1}{2}} \frac{\partial \gamma_{1 / 2}}{\partial \xi} C & A_{\frac{1}{2}} \frac{\partial \gamma_{1 / 2}}{\partial \xi} B
\end{array}\right)= \\
& \left(\begin{array}{cc}
A_{\frac{-1}{2}} & 0 \\
0 & A_{\frac{1}{2}}
\end{array}\right)\left(\begin{array}{cc}
\frac{\partial \gamma_{-1 / 2}}{\partial x} & \frac{\partial \gamma_{-1 / 2}}{\partial \xi} \\
\frac{\partial \gamma_{1 / 2}}{\partial x} & \frac{\partial \gamma_{1 / 2}}{\partial \xi}
\end{array}\right)\left(\begin{array}{cc}
B & 0 \\
0 & B
\end{array}\right)\left(\begin{array}{cc}
I & 0 \\
B^{-1} C & I
\end{array}\right) .
\end{aligned}
$$


Levando em conta a fórmula (B.3), os determinantes envolvidos são

$$
\begin{aligned}
\left|\operatorname{det}\left(\begin{array}{cc}
A_{\frac{-1}{2}} & 0 \\
0 & A_{\frac{1}{2}}
\end{array}\right)\right|=\left|\operatorname{det} A_{\frac{-1}{2}} \operatorname{det} A_{\frac{1}{2}}\right|=\sqrt{\frac{g\left(\gamma_{-1 / 2}\right)}{g^{\prime}\left(\gamma_{-1 / 2}^{\prime}\right)} \sqrt{\frac{g\left(\gamma_{1 / 2}\right)}{g^{\prime}\left(\gamma_{1 / 2}^{\prime}\right)}}} \\
\left|\operatorname{det}\left(\begin{array}{cc}
B & 0 \\
0 & B
\end{array}\right)\right|=|\operatorname{det} B|^{2}=\frac{g^{\prime}\left(x^{\prime}\right)}{g(x)},\left|\operatorname{det}\left(\begin{array}{cc}
I & 0 \\
B^{-1} C & I
\end{array}\right)\right|=1 .
\end{aligned}
$$

Assim,

$$
f^{\prime}=\sqrt{\frac{g\left(\gamma_{-1 / 2}\right)}{g^{\prime}\left(\gamma_{-1 / 2}^{\prime}\right)}} \sqrt{\frac{g\left(\gamma_{1 / 2}\right)}{g^{\prime}\left(\gamma_{1 / 2}^{\prime}\right)}} \frac{g^{\prime}\left(x^{\prime}\right)}{g(x)} f .
$$

Pelas definições (3.23) e (3.25) vemos que $\mathcal{M}$ se transforma como um escalar:

$$
\mathcal{M}(x, \xi)=\mathcal{M}^{\prime}\left(x^{\prime}, \xi^{\prime}\right)
$$

Se o espaço é plano, existe um sistema de coordenadas em que $g_{\mu \nu}$ é constante igual a $(2.17)$ e, portanto, $\Gamma_{\alpha \beta}^{\mu} \equiv 0$, o que implica $\gamma(x, \xi)=x+\xi$. Neste sistema de coordenadas, $g(x)=1 \mathrm{e}$

$$
\mathcal{M}(x, \xi)=f(x, \xi)=\left|\operatorname{det}\left(\begin{array}{cc}
I & -\frac{1}{2} I \\
I & \frac{1}{2} I
\end{array}\right)\right|=\left|\operatorname{det}\left(\begin{array}{cc}
2 I & 0 \\
I & \frac{1}{2} I
\end{array}\right)\right|=1 \text {. }
$$

Pela fórmula (3.27), $\mathcal{M}(x, \xi)=1$ em qualquer sistema de coordenadas.

Vamos considerar a expansão em potências de $\xi$ e $\xi^{\prime}$ de $\mathcal{M}(x, \xi)$ e $\mathcal{M}^{\prime}\left(x^{\prime}, \xi^{\prime}\right)$, usando a lei de transformação $\xi^{\prime \mu}=\frac{\partial x^{\prime \mu}}{\partial x^{\nu}} \xi^{\nu}$ :

$$
\begin{aligned}
\mathcal{M}(x, \xi) & =\sum_{n=0}^{\infty} \mathcal{M}_{\mu_{1} \cdots \mu_{n}}(x) \xi^{\mu_{1}} \ldots \xi^{\mu_{n}} \\
\mathcal{M}^{\prime}\left(x^{\prime}, \xi^{\prime}\right) & =\sum_{n=0}^{\infty} \mathcal{M}_{\mu_{1} \cdots \mu_{n}}^{\prime}\left(x^{\prime}\right) \xi^{\prime \mu_{1}} \ldots \xi^{\prime \mu_{n}} \\
& =\sum_{n=0}^{\infty} \mathcal{M}_{\mu_{1} \cdots \mu_{n}}^{\prime}\left(x^{\prime}\right) \frac{\partial x^{\prime \mu_{1}}}{\partial x^{\nu_{1}}} \cdots \frac{\partial x^{\prime \mu_{n}}}{\partial x^{\nu_{n}}} \xi^{\mu_{1}} \ldots \xi^{\mu_{n}}
\end{aligned}
$$

o que implica, pela igualdade (3.27)

$$
\mathcal{M}_{\mu_{1} \cdots \mu_{n}}(x)=\mathcal{M}_{\nu_{1} \cdots \nu_{n}}^{\prime}\left(x^{\prime}\right) \frac{\partial x^{\prime \nu_{1}}}{\partial x^{\mu_{1}}} \cdots \frac{\partial x^{\prime \nu_{n}}}{\partial x^{\mu_{n}}}
$$

ou seja, os coeficientes $\mathcal{M}_{\mu_{1} \cdots \mu_{n}}(x)$ são tensores. Isto permite calcular os primeiros termos da expansão em um sistema de coordenadas mais simples. Para tanto, o 
melhor sistema de coordenadas é o sistema de coordenadas normais (ver apêndice C.2). Se $x$ são coordenadas normais, então, pela fórmula (C.27),

$$
\gamma(0, \xi)=\xi \Leftrightarrow \gamma_{a}(0, \xi)=a \xi
$$

Esta propriedade implica que

$$
\frac{\partial \gamma_{a}^{\mu}}{\partial \xi^{\nu}}(0, \xi)=a \delta_{\nu}^{\mu}
$$

embora $\frac{\partial \gamma_{a}^{\mu}}{\partial x^{\nu}}(0, \xi) \neq \delta_{\nu}^{\mu}$ em geral, já que a fórmula (3.29) só vale no ponto $x=0$. Assim, nestas coordenadas,

$$
\begin{aligned}
& f(0, \xi)=\left|\operatorname{det}\left(\begin{array}{cc}
\frac{\partial \gamma_{-1 / 2}}{\partial x}(0, \xi) & \frac{\partial \gamma_{-1 / 2}}{\partial \xi}(0, \xi) \\
\frac{\partial \gamma_{1 / 2}}{\partial x}(0, \xi) & \frac{\partial \gamma_{1 / 2}}{\partial \xi}(0, \xi)
\end{array}\right)\right| \\
& =\left|\operatorname{det}\left(\begin{array}{cc}
\frac{\partial \gamma_{-1 / 2}}{\partial x}(0, \xi) & -\frac{1}{2} I \\
\frac{\partial \gamma_{1 / 2}}{\partial x}(0, \xi) & \frac{1}{2} I
\end{array}\right)\right| \\
& =\left|\operatorname{det}\left(\begin{array}{cc}
\frac{\partial \gamma_{-1 / 2}}{\partial x}(0, \xi)+\frac{\partial \gamma_{1 / 2}}{\partial x}(0, \xi) & 0 \\
\frac{\partial \gamma_{1 / 2}}{\partial x}(0, \xi) & \frac{1}{2} I
\end{array}\right)\right| \\
& =\frac{1}{2^{D}}\left|\operatorname{det}\left(\frac{\partial \gamma_{-1 / 2}}{\partial x}(0, \xi)+\frac{\partial \gamma_{1 / 2}}{\partial x}(0, \xi)\right)\right| \\
& =\left|\operatorname{det}\left(\frac{1}{2}\left(\frac{\partial \gamma_{-1 / 2}}{\partial x}(0, \xi)+\frac{\partial \gamma_{1 / 2}}{\partial x}(0, \xi)\right)\right)\right| \\
& =\left|\operatorname{det}\left(\frac{\partial}{\partial x}\left(\frac{\gamma\left(x,-\frac{1}{2} \xi\right)+\gamma\left(x, \frac{1}{2} \xi\right)}{2}\right)\right)\right|_{x=0} \text {. }
\end{aligned}
$$

Usando a expansão (C.25), temos

$$
\frac{\gamma^{\mu}\left(x,-\frac{1}{2} \xi\right)+\gamma^{\mu}\left(x, \frac{1}{2} \xi\right)}{2}=x^{\mu}-\frac{1}{8} \Gamma_{\alpha \beta}^{\mu}(x) \xi^{\alpha} \xi^{\beta}+O\left(\xi^{4}\right),
$$

e, portanto,

$$
\frac{\partial}{\partial x^{\nu}}\left(\frac{\gamma^{\mu}\left(x,-\frac{1}{2} \xi\right)+\gamma^{\mu}\left(x, \frac{1}{2} \xi\right)}{2}\right)=\delta_{\nu}^{\mu}-\frac{1}{8} \frac{\partial \Gamma_{\alpha \beta}^{\mu}}{\partial x^{\nu}}(x) \xi^{\alpha} \xi^{\beta}+O\left(\xi^{4}\right)
$$

Pela fórmula $\operatorname{det}(I+A)=1+\operatorname{tr} A+O\left(A^{2}\right)$, temos

$$
f(0, \xi)=1-\frac{1}{8} \frac{\partial \Gamma_{\alpha \beta}^{\mu}}{\partial x^{\mu}}(0) \xi^{\alpha} \xi^{\beta}+O\left(\xi^{4}\right) .
$$


Por (3.29), temos

$$
\begin{aligned}
\frac{\sqrt{g\left(\gamma_{a}(0, \xi)\right)}}{\sqrt{g(0)}} & =\frac{\sqrt{g(a \xi)}}{\sqrt{g(x)}} \\
& =1+\left.a \xi^{\alpha} \frac{1}{\sqrt{g}} \frac{\partial \sqrt{g}}{\partial x^{\alpha}}\right|_{x=0}+\left.\frac{a^{2}}{2} \xi^{\alpha} \xi^{\beta} \frac{1}{\sqrt{g}} \frac{\partial^{2} \sqrt{g}}{\partial x^{\alpha} \partial x^{\beta}}\right|_{x=0}+O\left(\xi^{3}\right) .
\end{aligned}
$$

Levando em conta a fórmula (B.2) e que em coordenadas normais a conexão de Levi-Civita é nula

$$
\begin{aligned}
\frac{\sqrt{g\left(\gamma_{a}(0, \xi)\right)}}{\sqrt{g(x)}} & =1+\frac{a^{2}}{2} \xi^{\alpha} \xi^{\beta} \frac{1}{\sqrt{g}} \frac{\partial}{\partial x^{\alpha}}\left(\sqrt{g} \frac{1}{\sqrt{g}} \frac{\partial \sqrt{g}}{\partial x^{\beta}}\right)_{x=0}+O\left(\xi^{3}\right) \\
& =1+\frac{a^{2}}{2} \xi^{\alpha} \xi^{\beta} \frac{\partial \Gamma_{\beta}}{\partial x^{\alpha}}(0)+O\left(\xi^{3}\right) .
\end{aligned}
$$

Assim, vale a expansão ${ }^{2}$

$$
\begin{aligned}
\frac{\sqrt{g\left(\gamma_{-1 / 2}(0, \xi)\right) g\left(\gamma_{1 / 2}(0, \xi)\right)}}{g(x)} & =\left(1+\frac{1}{8} \xi^{\alpha} \xi^{\beta} \frac{\partial \Gamma_{\beta}}{\partial x^{\alpha}}(0)\right)^{2}+O\left(\xi^{4}\right) \\
& =1+\frac{1}{4} \xi^{\alpha} \xi^{\beta} \frac{\partial \Gamma_{\beta}}{\partial x^{\alpha}}(0)+O\left(\xi^{4}\right) .
\end{aligned}
$$

Juntando (3.30) e (3.31), temos

$$
\begin{aligned}
\mathcal{M}(0, \xi) & =\left(1+\frac{1}{4} \xi^{\alpha} \xi^{\beta} \frac{\partial \Gamma_{\beta}}{\partial x^{\alpha}}(0)\right)\left(1-\frac{1}{8} \frac{\partial \Gamma_{\alpha \beta}^{\mu}}{\partial x^{\mu}}(0) \xi^{\alpha} \xi^{\beta}\right)+O\left(\xi^{4}\right) \\
& =1-\left(\frac{1}{8} \frac{\partial \Gamma_{\alpha \beta}^{\mu}}{\partial x^{\mu}}(0)-\frac{1}{4} \frac{\partial \Gamma_{\beta}}{\partial x^{\alpha}}(0)\right) \xi^{\alpha} \xi^{\beta}+O\left(\xi^{4}\right) .
\end{aligned}
$$

Pela fórmula (C.30), temos

$$
\mathcal{M}(0, \xi)=1-\frac{1}{6} R_{\alpha \beta}(0) \xi^{\alpha} \xi^{\beta}+O\left(\xi^{4}\right)
$$

onde $R_{\alpha \beta}$ é o tensor de Ricci (B.7). Dado que os coeficientes da expansão de $\mathcal{M}$ são tensores, ou seja, se transformam pela lei (3.28), a fórmula acima vale em qualquer referencial. O ponto 0 em coordenadas normais corresponde a qualquer ponto do espaço, logo em qualquer ponto $x$ e em qualquer referencial

$$
\mathcal{M}(x, \xi)=1-\frac{1}{6} R_{\alpha \beta}(x) \xi^{\alpha} \xi^{\beta}+O\left(\xi^{4}\right) .
$$

${ }^{2} \mathrm{~A}$ precisão é $O\left(\xi^{4}\right)$ porque a função $\frac{\sqrt{g\left(\gamma_{-1 / 2}(x, \xi)\right) g\left(\gamma_{1 / 2}(x, \xi)\right)}}{g(x)}$ é par em $\xi$. 


\subsection{Forma geral de $Q_{\omega}$ em espaços curvos}

A fórmula (3.11), junto com a observação da seção anterior de que em um espaço plano $\mathcal{M}(x, \xi)=1$, mostra que em um espaço plano

$$
\left\langle\left. y\right|_{\omega^{1}} Q_{\omega}(F) \mid z\right\rangle_{\omega^{1}}=\int \widetilde{\left(\frac{F}{\sqrt{g}}\right)}\left(T_{\theta}(\bar{x}, \bar{\xi})\right) \omega(\theta) d \theta .
$$

Assim, podemos considerar uma generalização de $Q_{\omega}$ a um espaço curvo qualquer correspondência $Q_{\omega, \mathcal{F}}$ cujo elemento de matriz seja dado por

$$
\left\langle\left. y\right|_{\omega^{1}} Q_{\omega, \mathcal{F}}(F) \mid z\right\rangle_{\omega^{1}}=\frac{\mathcal{F}(\bar{x}, \bar{\xi})}{\mathcal{M}(\bar{x}, \bar{\xi})} \int \widetilde{\left(\frac{F}{\sqrt{g}}\right)}\left(T_{\theta}(\bar{x}, \bar{\xi})\right) \omega(\theta) d \theta
$$

onde a função $\mathcal{F}(x, \xi)$ obedece a

1. $\mathcal{F}(x, \xi)=1$ se $R_{\mu \nu \alpha \beta}=0$ (ou seja, se o espaço é plano)

2. $\mathcal{F}$ é um escalar quando definido em diversos sistemas de coordenadas, isto é, $\mathcal{F}(x, \xi)=\mathcal{F}\left(x^{\prime}, \xi^{\prime}\right)$ (equação 3.27 ) pelas transformações 3.24

3. $\mathcal{F}(x, 0)=1$

4. $\mathcal{F}(x, \xi)$ é real e par em $\xi(\mathcal{F}(x, \xi)=\mathcal{F}(x,-\xi))$.

A propriedade 1 implica que o elemento de matriz (3.33) é idêntico ao elemento de matriz de $Q_{\omega}(F)$ se o espaço é plano e, portanto, $Q_{\omega, \mathcal{F}}$ pode ser considerada uma generalização de $Q_{\omega}$. A propriedade 2 é necessária para garantir a covariância de $Q_{\omega, \mathcal{F}}$. As propriedades 3 e 4 serão necessárias para demonstrar propriedades básicas de $Q_{\omega, \mathcal{F}}(F)$ e o limite clássico.

O operador $Q_{\omega, \mathcal{F}}(F)$ pode ser expresso levando em conta a normalização (2.64) como

$$
Q_{\omega, \mathcal{F}}(F)=\int\left\langle\left. y\right|_{\omega^{1}} Q_{\omega, \mathcal{F}}(F) \mid z\right\rangle_{\omega^{1}}|y\rangle_{\omega^{1}}\left\langle\left. z\right|_{\omega^{1}} \sqrt{g(y)} d y \sqrt{g(z)} d z\right.
$$

A definição de $Q_{\omega, \mathcal{F}}$ contém a definição de $Q_{\omega, 1}$. De fato, $Q_{\omega, 1}=Q_{\omega, \mathcal{F}}$ quando $\mathcal{F}$ é a função constante $\mathcal{F}(x, \xi)=1$.

A generalização da quantização de Weyl se define usando $\omega(\theta)=\delta(\theta)$, da mesma maneira que para a dedução de equação (3.12):

$$
\left\langle\left. y\right|_{0} Q^{\mathrm{W}, \mathcal{F}}(F) \mid z\right\rangle_{0}=\frac{\mathcal{F}(\bar{x}, \bar{\xi})}{\mathcal{M}(\bar{x}, \bar{\xi})} \widetilde{\left(\frac{F}{\sqrt{g}}\right)}(\bar{x}, \bar{\xi})=\frac{\mathcal{F}(\bar{x}, \bar{\xi})}{\mathcal{M}(\bar{x}, \bar{\xi})} \frac{\tilde{F}(\bar{x}, \bar{\xi})}{\sqrt{g(\bar{x})}} .
$$


Dado um operador $\hat{F}$, definimos o seu $\omega, \mathcal{F}$-símbolo como sendo a função $F_{\omega, \mathcal{F}}(x, p)$ tal que $\hat{F}=Q_{\omega, \mathcal{F}}\left(F_{\omega, \mathcal{F}}\right)$ e o seu segundo símbolo de Weyl como sendo a função $F^{\mathrm{W}, \mathcal{F}}(x, p)$ tal que $\hat{F}=Q^{\mathrm{W}, \mathcal{F}}\left(F^{\mathrm{W}, \mathcal{F}}\right)$, da mesma maneira que na seção 3.4. Uma comparação entre as fórmulas (3.33), (3.35) e (3.11), (3.12) mostra que a relação entre $F_{\omega, \mathcal{F}}$ e $F^{\mathrm{W}, \mathcal{F}}$ é a mesma que entre $F_{\omega, 1}$ e $F^{\mathrm{W}, 1}$, isto é,

$$
\begin{aligned}
F^{\mathrm{W}, \mathcal{F}}(x, p) & =\Lambda_{\omega^{1}}\left(x,-i \hbar \frac{\partial}{\partial p}\right) \sum_{n=0}^{\infty} \frac{\omega^{n}}{n !}(-\hbar \Delta)^{n} F_{\omega, \mathcal{F}}(x, p) \\
& =\Lambda_{\omega^{1}}\left(x,-i \hbar \frac{\partial}{\partial p}\right) \Omega(-\hbar \Delta) F_{\omega, \mathcal{F}}(x, p) .
\end{aligned}
$$

As equações (3.12) e (3.35) mostram que existe a relação $\widetilde{F^{\mathrm{W}, 1}}(x, \xi)=\mathcal{F}(x, \xi) \widetilde{F^{\mathrm{W}, \mathcal{F}}}(x, \xi)$, ou seja,

$$
F^{\mathrm{W}, 1}(x, \xi)=\mathcal{F}\left(x,-i \hbar \frac{\partial}{\partial p}\right) F^{\mathrm{W}, \mathcal{F}}(x, \xi)
$$

O primeiro símbolo de Weyl pode então ser expresso em termos do símbolo $F_{\omega, \mathcal{F}}$ como

$$
F^{\mathrm{W}, 1}(x, \xi)=\mathcal{F}\left(x,-i \hbar \frac{\partial}{\partial p}\right) \Lambda_{\omega^{1}}\left(x,-i \hbar \frac{\partial}{\partial p}\right) \Omega(-\hbar \Delta) F_{\omega, \mathcal{F}}(x, p)
$$

Pela igualdade (3.22), podemos expressar $F_{\omega, 1}$ através de $F_{\omega, \mathcal{F}}$ :

$$
\begin{aligned}
F_{\omega, 1}(x, p)=[\Omega(-\hbar \Delta)]^{-1} & {\left[\Lambda_{\omega^{1}}\left(x,-i \hbar \frac{\partial}{\partial p}\right)\right]^{-1} \times } \\
& \times \mathcal{F}\left(x,-i \hbar \frac{\partial}{\partial p}\right) \Lambda_{\omega^{1}}\left(x,-i \hbar \frac{\partial}{\partial p}\right) \Omega(-\hbar \Delta) F_{\omega, \mathcal{F}}(x, p) .
\end{aligned}
$$

O operador $\Lambda_{\omega^{1}}\left(x,-i \hbar \frac{\partial}{\partial p}\right)$ comuta com $\mathcal{F}\left(x,-i \hbar \frac{\partial}{\partial p}\right)$; logo temos a expressão

$$
F_{\omega, 1}(x, p)=[\Omega(-\hbar \Delta)]^{-1} \mathcal{F}\left(x,-i \hbar \frac{\partial}{\partial p}\right) \Omega(-\hbar \Delta) F_{\omega, \mathcal{F}}(x, p)
$$

A igualdade $Q_{\omega, \mathcal{F}}\left(F_{\omega, \mathcal{F}}\right)=Q_{\omega, 1}\left(F_{\omega, 1}\right)$ permite então expressar concretamente $Q_{\omega, \mathcal{F}}$ como

$$
Q_{\omega, \mathcal{F}}(F)=Q_{\omega, 1}\left([\Omega(-\hbar \Delta)]^{-1} \mathcal{F}\left(x,-i \hbar \frac{\partial}{\partial p}\right) \Omega(-\hbar \Delta) F\right)
$$

Esta expressão permite demonstrar as propriedades básicas de $Q_{\omega, \mathcal{F}}$. O operador $\Omega(-\hbar \Delta)$ é real, pois $\Delta$ é real e as equações $(2.29)$ e (2.25) implicam que a função $\Omega(k)$ é real, e $\mathcal{F}\left(x,-i \hbar \frac{\partial}{\partial p}\right)$ também é real, pois $\mathcal{F}(x, \xi)$ é par em $\xi$. Assim, se $F$ é 
real, $[\Omega(-\hbar \Delta)]^{-1} \mathcal{F}\left(x,-i \hbar \frac{\partial}{\partial p}\right) \Omega(-\hbar \Delta) F$ também é, o que implica, pela equação (3.38) e pela hermiticidade de $Q_{\omega, 1}$, que $Q_{\omega, \mathcal{F}}(F)$ é um operador auto-adjunto. Assim, $Q_{\omega, \mathcal{F}}$ é uma quantização auto-adjunta.

Seja $F=F(x)$ uma função apenas de $x$. Temos $\frac{\partial}{\partial p} F=0$ e, pela definição (3.18), $\Delta F=0$. Pela condição $(2.27)$, temos $\Omega(0)=1$. Pela propriedade $\mathcal{F}(x, 0)=1$,

$$
[\Omega(-\hbar \Delta)]^{-1} \mathcal{F}\left(x,-i \hbar \frac{\partial}{\partial p}\right) \Omega(-\hbar \Delta) F=[\Omega(0)]^{-1} \mathcal{F}(x, 0) \Omega(0) F=F .
$$

Portanto, pela equação $(3.38), Q_{\omega, \mathcal{F}}(F)=Q_{\omega, 1}(F)=F(\hat{x})$. Isto implica em particular que $Q_{\omega, \mathcal{F}}(1)=\hat{I}$ e $Q_{\omega, \mathcal{F}}\left(x^{\mu}\right)=\hat{x}^{\mu}$.

Consideremos um polinômio $T^{\mu}(x) p_{\mu}$ de grau 1 em $p$. Pela definição (3.18), temos que $\Omega(-\hbar \Delta)\left(T^{\mu}(x) p_{\mu}\right)$ é também um polinômio de grau 1 em $p$. A estrutura $\mathcal{F}(x, \xi)=1+O\left(\xi^{2}\right)$ implica então que

$$
\begin{aligned}
{[\Omega(-\hbar \Delta)]^{-1} \mathcal{F}\left(x,-i \hbar \frac{\partial}{\partial p}\right) \Omega(-\hbar \Delta)\left(T^{\mu}(x) p_{\mu}\right) } & \\
& =[\Omega(-\hbar \Delta)]^{-1} \Omega(-\hbar \Delta)\left(T^{\mu}(x) p_{\mu}\right)=T^{\mu}(x) p_{\mu}
\end{aligned}
$$

Assim, pela equação (3.38),

$$
Q_{\omega, \mathcal{F}}\left(T^{\mu}(x) p_{\mu}\right)=Q_{\omega, 1}\left(T^{\mu}(x) p_{\mu}\right)
$$

em particular,

$$
Q_{\omega, \mathcal{F}}\left(p_{\mu}\right)=Q_{\omega, 1}\left(p_{\mu}\right)=\hat{p}_{\mu}
$$

\subsection{Covariância de $Q_{\omega, \mathcal{F}}$}

Pela definição da quantização $Q_{\omega, \mathcal{F}}(F)(3.33)$ e do fluxo geodésico (C.13), temos

$$
\left\langle y\left|Q_{\omega, \mathcal{F}}(F)\right| z\right\rangle=\frac{\mathcal{F}(\bar{x}, \bar{\xi})}{\mathcal{M}(\bar{x}, \bar{\xi})} \int \widetilde{\left(\frac{F}{\sqrt{g}}\right)}\left(\gamma(\bar{x}, \theta \bar{\xi}), \frac{1}{\theta} \beta(\bar{x}, \theta \bar{\xi})\right) \omega(\theta) d \theta
$$

onde $\bar{x}$ e $\bar{\xi}$ são o ponto médio e o vetor tangente à geodésica mínima que une os pontos $y$ e $z$. Pela lei de transformação (2.43), a equação acima fica

$$
\left\langle y\left|Q_{\omega, \mathcal{F}}(F)\right| z\right\rangle=\frac{\mathcal{F}(\bar{x}, \bar{\xi})}{\mathcal{M}(\bar{x}, \bar{\xi})} \int \widetilde{\left(\frac{F^{\prime}}{\sqrt{g^{\prime}}}\right)}\left(\varphi(\gamma(\bar{x}, \theta \bar{\xi})), \frac{\partial x^{\prime}}{\partial x}(\gamma(\bar{x}, \theta \bar{\xi})) \frac{1}{\theta} \beta(\bar{x}, \theta \bar{\xi})\right) \omega(\theta) d \theta .
$$


Pelas leis de transformação (C.17), (C.18) da função exponencial, e pela lei de transformação escalar 3.27 à qual $\mathcal{F}$ também obedece,

$$
\begin{aligned}
\left\langle y\left|Q_{\omega, \mathcal{F}}(F)\right| z\right\rangle & =\frac{\mathcal{F}^{\prime}\left(\bar{x}^{\prime}, \bar{\xi}^{\prime}\right)}{\mathcal{M}^{\prime}\left(\bar{x}^{\prime}, \bar{\xi}^{\prime}\right)} \int \widetilde{\left(\frac{F^{\prime}}{\sqrt{g^{\prime}}}\right)}\left(\gamma^{\prime}\left(\bar{x}^{\prime}, \theta \bar{\xi}^{\prime}\right), \frac{1}{\theta} \beta^{\prime}\left(\bar{x}^{\prime}, \theta \bar{\xi}^{\prime}\right)\right) \omega(\theta) d \theta \\
& =\frac{\mathcal{F}^{\prime}\left(\bar{x}^{\prime}, \bar{\xi}^{\prime}\right)}{\mathcal{M}^{\prime}\left(\bar{x}^{\prime}, \bar{\xi}^{\prime}\right)} \int \widehat{\left(\frac{F^{\prime}}{\sqrt{g^{\prime}}}\right)}\left(T_{\theta}^{\prime}\left(\bar{x}^{\prime}, \bar{\xi}^{\prime}\right)\right) \omega(\theta) d \theta
\end{aligned}
$$

$\operatorname{com} \bar{x}^{\prime}=\varphi(\bar{x})$ e $\bar{\xi}^{\prime}=\frac{\partial x^{\prime}}{\partial x}(\bar{x}) \xi$. Pela lei de transformação (C.17),

$$
\begin{aligned}
\gamma^{\prime}\left(\bar{x}^{\prime}, \frac{-1}{2} \bar{\xi}^{\prime}\right) & =\varphi\left(\gamma\left(\bar{x}, \frac{-1}{2} \bar{\xi}\right)\right)=\varphi(y)=y^{\prime} \\
\gamma^{\prime}\left(\bar{x}^{\prime}, \frac{1}{2} \bar{\xi}^{\prime}\right) & =\varphi\left(\gamma\left(\bar{x}, \frac{1}{2} \bar{\xi}\right)\right)=\varphi(z)=z^{\prime}
\end{aligned}
$$

e, portanto, $\bar{x}^{\prime}$ e $\bar{\xi}^{\prime}$ são o ponto médio e o vetor tangente à geodésica que une $y^{\prime}$ a $z^{\prime}$, no sistema de coordenadas $x^{\prime}=\varphi(x)$. Assim, o último membro da equação (3.40) é o elemento de matriz (3.33) nas coordenadas $x^{\prime}$. Temos, portanto, a igualdade

$$
\left\langle y\left|Q_{\omega, \mathcal{F}}(F)\right| z\right\rangle=\left\langle\left. y^{\prime}\right|^{\prime} Q_{\omega, \mathcal{F}}^{\prime}\left(F^{\prime}\right) \mid z^{\prime}\right\rangle^{\prime}
$$

Precisamos agora determinar a forma das equações (1.8) e (1.9) para a quantização $Q_{\omega, \mathcal{F}}$, que definem a transformação linear $U: \mathcal{R} \rightarrow \mathcal{R}^{\prime}$. A propriedade $Q_{\omega, \mathcal{F}}(F(x))=F(\hat{x})$ determinada na seção anterior mostra que (1.8) tem a mesma forma (3.3). Pela equação (3.39), vemos que continua valendo a forma (3.4) para (1.9). Isto mostra que, assim como na seção (3.2), se os vetores $|x\rangle \in \mathcal{R}$ obedecem a (2.64) e (2.65), então os vetores $\left|x^{\prime}\right\rangle^{\prime}=|\varphi(x)\rangle^{\prime}=U|x\rangle \in \mathcal{R}^{\prime}$ também as obedecerão, no sistema de coordenadas $x^{\prime}=\varphi(x)$.

Podemos agora calcular $U Q_{\omega, \mathcal{F}}(F) U^{\dagger}$ usando a expressão (3.34), usando a relação $U|x\rangle=|\varphi(x)\rangle^{\prime}$ :

$$
\begin{aligned}
U Q_{\omega, \mathcal{F}}(F) U^{\dagger} & =\int\left\langle y\left|Q_{\omega, \mathcal{F}}(F)\right| z\right\rangle|\varphi(y)\rangle^{\prime}\left\langle\left.\varphi(z)\right|^{\prime} \sqrt{g(y)} d y \sqrt{g(z)} d z\right. \\
& =\int\left\langle\left. y^{\prime}\right|^{\prime} Q_{\omega, \mathcal{F}}^{\prime}\left(F^{\prime}\right) \mid z^{\prime}\right\rangle^{\prime}\left|y^{\prime}\right\rangle^{\prime}\left\langle\left. z^{\prime}\right|^{\prime} \sqrt{g^{\prime}\left(y^{\prime}\right)} d y^{\prime} \sqrt{g^{\prime}\left(z^{\prime}\right)} d z^{\prime}\right. \\
& =Q_{\omega, \mathcal{F}}^{\prime}\left(F^{\prime}\right),
\end{aligned}
$$

onde fizemos a mudança de variáveis $y^{\prime}=\varphi(y)$ e $z^{\prime}=\varphi\left(z^{\prime}\right)$ na integral, levando em conta a transformação do elemento de volume (B.4). Esta equação mostra que $Q_{\omega, \mathcal{F}}$ é uma quantização covariante. 


\subsection{Produto de símbolos e limite clássico}

Pela definição (3.35), o elemento de matriz de um operador $\hat{F}=Q^{\mathrm{W}, \mathcal{M}}(F)$ se relaciona com o seu $\mathcal{M}$-símbolo de Weyl $F$ por

$$
\langle y|\hat{F}| z\rangle=\widetilde{F_{g}}(\bar{x}(y, z), \bar{\xi}(y, z))
$$

onde definimos $F_{g}=F / \sqrt{g}$. O produto $*_{\mathrm{W}, \mathcal{M}}$ de símbolos por esta quantização é tal que $Q^{\mathrm{W}, \mathcal{M}}(F * \mathrm{w}, \mathcal{M} G)=\hat{F} \hat{G}$, logo

$$
\langle y|\hat{F} \hat{G}| z\rangle=\left(\left(F *_{\mathrm{W}, \mathcal{M}} G\right)_{g}\right)^{\sim}(\bar{x}(y, z), \bar{\xi}(y, z)) .
$$

Pela normalização (2.64), a completeza dos estados $|x\rangle$ implica em

$$
\langle y|\hat{F} \hat{G}| z\rangle=\int\langle y|\hat{F}| w\rangle\langle w|\hat{G}| z\rangle \sqrt{g(w)} d w
$$

Assim, podemos expressar o produto de símbolos $*_{\mathrm{W}, \mathcal{M}}$ por

$$
\begin{aligned}
& \left(\left(F *_{\mathrm{W}, \mathcal{M}} G\right)_{\sqrt{g}}\right)^{\sim}(\bar{x}(y, z), \bar{\xi}(y, z))= \\
& \qquad \widetilde{F_{g}}(\bar{x}(y, w), \bar{\xi}(y, w)) \widetilde{G_{g}}(\bar{x}(w, z), \bar{\xi}(w, z)) \sqrt{g(w)} d w
\end{aligned}
$$

Queremos expressar o produto ${ }^{*} \mathrm{~W}, \mathcal{M}$ até a ordem de $\hbar$. Sejam $x=\bar{x}(y, z)$ e $\xi=\bar{\xi}(y, z)$. Temos $y=\gamma\left(x, \frac{-1}{2} \xi\right)$ e $z=\gamma\left(x, \frac{1}{2} \xi\right)$. Pela expansão (C.25),

$$
\begin{aligned}
c^{\mu} & =\frac{y^{\mu}+z^{\mu}}{2}=x^{\mu}-\frac{1}{8} \Gamma_{\mu_{1} \mu_{2}}^{\mu}(x) \xi^{\mu_{1}} \xi^{\mu_{2}}+O\left(\xi^{4}\right), \\
l^{\mu} & =z^{\mu}-y^{\mu}=\xi^{\mu}+\frac{1}{48} \Gamma_{\mu_{1} \mu_{2} \mu_{3}}^{\mu}(x) \xi^{\mu_{1}} \xi^{\mu_{2}} \xi^{\mu_{3}}+O\left(\xi^{5}\right) .
\end{aligned}
$$

Usando (3.42), para uma função qualquer $A(x, p)$, temos

$$
\begin{aligned}
\tilde{A}(x, \xi) & =\tilde{A}(c, \xi)+\frac{1}{8} \Gamma_{\mu_{1} \mu_{2}}^{\mu}(x) \xi^{\mu_{1}} \xi^{\mu_{2}} \frac{\widetilde{\partial A}}{\partial x^{\mu}}(c, \xi)+O\left(\xi^{4}\right) \\
& =\tilde{A}(c, \xi)-\frac{\hbar^{2}}{8}\left(\Gamma_{\mu_{1} \mu_{2}}^{\mu}(x) \frac{\partial^{3} A}{\partial p_{\mu_{1}} \partial p_{\mu_{2}} \partial x^{\mu}}\right)^{\sim}(c, \xi)+O\left(\hbar^{4}\right)
\end{aligned}
$$

A relação (3.43) entre $l^{\mu}$ e $\xi^{\mu}$ pode ser invertida como

$$
\xi^{\mu}=l^{\mu}-\frac{1}{48} \Gamma_{\mu_{1} \mu_{2} \mu_{3}}^{\mu}(x) l^{\mu_{1}} l^{\mu_{2}} l^{\mu_{3}}+O\left(l^{5}\right),
$$


o que implica

$$
\begin{aligned}
\tilde{A}(c, \xi) & =\tilde{A}(c, l)-\frac{1}{48} \Gamma_{\mu_{1} \mu_{2} \mu_{3}}^{\mu}(x) l^{\mu_{1}} l^{\mu_{2}} l^{\mu_{3}} \frac{\partial \tilde{A}}{\partial \xi^{\mu}}(c, l)+O\left(l^{5}\right) \\
& =\tilde{A}(c, l)+\frac{i}{\hbar} \frac{1}{48} \Gamma_{\mu_{1} \mu_{2} \mu_{3}}^{\mu}(x) l^{\mu_{1}} l^{\mu_{2}} l^{\mu_{3}}\left(p_{\mu} A\right)^{\sim}(c, l)+O\left(l^{5}\right) \\
& =\tilde{A}(c, l)-\frac{\hbar^{2}}{48}\left(\Gamma_{\mu_{1} \mu_{2} \mu_{3}}^{\mu}(x) \frac{\partial^{3}\left(p_{\mu} A\right)}{\partial p_{\mu_{1}} \partial p_{\mu_{2}} \partial p_{\mu_{3}}}\right)^{\sim}(c, l)+O\left(\hbar^{4}\right) .
\end{aligned}
$$

Temos, assim, o resultado

$$
\tilde{A}(\bar{x}(y, z), \bar{\xi}(y, z))=\tilde{A}\left(\frac{y+z}{2}, z-y\right)+O\left(\hbar^{2}\right)
$$

Com as definições $x=\frac{y+z}{2}$ e $\xi=z-y$, podemos agora escrever a equação (3.41) como

$$
\begin{aligned}
\left(\left(F *_{\mathrm{W}, \mathcal{M}} G\right)_{\sqrt{g}}\right)^{\sim}(x, \xi)= & \int \widetilde{F_{g}}\left(\frac{y+w}{2}, w-y\right) \widetilde{G_{g}}\left(\frac{w+z}{2}, z-w\right) \sqrt{g(w)} d w+O\left(\hbar^{2}\right) \\
= & \int \widetilde{F_{g}}\left(x-\frac{z-w}{2}, w-y\right) \widetilde{G_{g}}\left(x+\frac{w-y}{2}, z-w\right) \sqrt{g(w)} d w \\
& +O\left(\hbar^{2}\right) .
\end{aligned}
$$

Chamando temporariamente $\eta=w-y, \zeta=z-w$, o integrando é igual a

$$
\begin{aligned}
& \widetilde{F_{g}}\left(x-\frac{\zeta}{2}, \eta\right) \widetilde{G_{g}}\left(x+\frac{\eta}{2}, \zeta\right)= \\
& \quad\left(\widetilde{F_{g}}(x, \eta)-\frac{\zeta^{\mu}}{2} \frac{\partial \widetilde{F_{g}}}{\partial x^{\mu}}(x, \eta)+O\left(\zeta^{2}\right)\right)\left(\widetilde{G_{g}}(x, \zeta)+\frac{\eta^{\mu}}{2} \frac{\partial \widetilde{G_{g}}}{\partial x^{\mu}}(x, \zeta)+O\left(\eta^{2}\right)\right) .
\end{aligned}
$$

As igualdades

$$
\zeta^{\mu} \widetilde{G_{g}}(x, \zeta)=-i \hbar \frac{\widetilde{\partial G_{g}}}{\partial p_{\mu}}(x, \zeta), \eta^{\mu} \widetilde{F_{g}}(x, \eta)=-i \hbar \frac{\widetilde{\partial F_{g}}}{\partial p_{\mu}}(x, \eta)
$$

implicam que

$$
\begin{aligned}
\widetilde{F_{g}}\left(x-\frac{\zeta}{2}, \eta\right) \widetilde{G_{g}}\left(x+\frac{\eta}{2}, \zeta\right)=\widetilde{F_{g}}(x, \eta) \widetilde{G_{g}}(x, \zeta) \\
+\frac{i \hbar}{2}\left(\frac{\widetilde{\partial F_{g}}}{\partial x^{\mu}}(x, \eta) \frac{\widetilde{\partial G_{g}}}{\partial p_{\mu}}(x, \zeta)-\frac{\widetilde{\partial F_{g}}}{\partial p_{\mu}}(x, \eta) \frac{\partial \widetilde{G_{g}}}{\partial x^{\mu}}(x, \zeta)\right)+O\left(\hbar^{2}\right) .
\end{aligned}
$$


Pela definição $F_{g}=F / \sqrt{g}$ e pela fórmula (B.2), temos

$$
\frac{\partial F_{g}}{\partial x^{\mu}}=\frac{\partial}{\partial x^{\mu}}\left(\frac{1}{\sqrt{g}} F\right)=\frac{1}{\sqrt{g}}\left(\frac{\partial F}{\partial x^{\mu}}-\Gamma_{\mu} F\right),
$$

e, portanto,

$$
\begin{aligned}
& \widetilde{F_{g}}\left(x-\frac{\zeta}{2}, \eta\right) \widetilde{G_{g}}\left(x+\frac{\eta}{2}, \zeta\right)=\frac{1}{g(x)} \widetilde{F}(x, \eta) \widetilde{G}(x, \zeta)+\frac{i \hbar}{2 g(x)} \times \\
& \times\left(\left(\frac{\partial F}{\partial x^{\mu}}-\Gamma_{\mu} F\right)^{\sim}(x, \eta) \frac{\widetilde{\partial G}}{\partial p_{\mu}}(x, \zeta)-\frac{\widetilde{\partial F}}{\partial p_{\mu}}(x, \eta)\left(\frac{\partial G}{\partial x^{\mu}}-\Gamma_{\mu} G\right)^{\sim}(x, \zeta)\right)+O\left(\hbar^{2}\right) \\
& =\frac{1}{g(x)} \widetilde{F}(x, \eta) \widetilde{G}(x, \zeta) \\
& +\frac{i \hbar}{2 g(x)}\left(\frac{\widetilde{\partial F}}{\partial x^{\mu}}(x, \eta) \frac{\widetilde{\partial G}}{\partial p_{\mu}}(x, \zeta)-\frac{\widetilde{\partial F}}{\partial p_{\mu}}(x, \eta) \frac{\widetilde{\partial G}}{\partial x^{\mu}}(x, \zeta)\right) \\
& +\frac{i \hbar}{2 g(x)} \Gamma_{\mu}(x)\left(\frac{\partial F}{\partial p_{\mu}}(x, \eta) \tilde{G}(x, \zeta)-\tilde{F}(x, \eta) \frac{\widetilde{\partial G}}{\partial p_{\mu}}(x, \zeta)\right)+O\left(\hbar^{2}\right)
\end{aligned}
$$

Assim, a integral (3.44) se decompõe como uma soma de 3 integrais

$$
\begin{gathered}
\left((F * \mathrm{~W}, \mathcal{M} G)_{\sqrt{g}}\right)^{\sim}(x, \xi)=I_{1}+I_{2}+I_{3}+O\left(\hbar^{2}\right) \\
I_{1}=\frac{1}{g(x)} \int \widetilde{F}(x, \eta) \widetilde{G}(x, \zeta) \sqrt{g(w)} d w \\
I_{2}=\frac{i \hbar}{2 g(x)} \int\left(\frac{\partial F}{\partial x^{\mu}}(x, \eta) \frac{\widetilde{\partial G}}{\partial p_{\mu}}(x, \zeta)-\frac{\widetilde{\partial F}}{\partial p_{\mu}}(x, \eta) \frac{\widetilde{\partial G}}{\partial x^{\mu}}(x, \zeta)\right) \sqrt{g(w)} d w \\
I_{3}=\frac{i \hbar}{2 g(x)} \Gamma_{\mu}(x) \int\left(\frac{\widetilde{\partial F}}{\partial p_{\mu}}(x, \eta) \tilde{G}(x, \zeta)-\tilde{F}(x, \eta) \frac{\widetilde{\partial G}}{\partial p_{\mu}}(x, \zeta)\right) \sqrt{g(w)} d w
\end{gathered}
$$

Nestas integrais, $F$ e suas derivadas são calculadas em $(x, \eta)$ e $G$ e suas derivadas são calculadas em $(x, \zeta)$. Vemos assim que as três integrais $I_{1}, I_{2}, I_{1}$, são convoluções da mesma forma

$$
\int \widetilde{F}(x, \eta) \widetilde{G}(x, \zeta) \sqrt{g(w)} d w=\int \widetilde{F}(x, w-y) \widetilde{G}(x, z-w) \sqrt{g(w)} d w
$$

Estamos lidando apenas com funções polinomiais de $(x, p)$. Assim,

$$
\widetilde{F}(x, w-y)=\left.F\left(x, i \hbar \frac{\partial}{\partial k}\right) \delta(w-y+k)\right|_{k=0}
$$


e, portanto,

$$
\begin{aligned}
& \int \widetilde{F}(x, \eta) \widetilde{G}(x, \zeta) \sqrt{g(w)} d w \\
& =\left.F\left(x, i \hbar \frac{\partial}{\partial k_{1}}\right) G\left(x, i \hbar \frac{\partial}{\partial k_{2}}\right) \int \delta\left(w-y+k_{1}\right) \delta\left(z-w+k_{2}\right) \sqrt{g(w)} d w\right|_{k_{1}=k_{2}=0} \\
& =\left.F\left(x, i \hbar \frac{\partial}{\partial k_{1}}\right) G\left(x, i \hbar \frac{\partial}{\partial k_{2}}\right) \delta\left(\xi+k_{1}+k_{2}\right) \sqrt{g\left(z+k_{2}\right)}\right|_{k_{1}=k_{2}=0} .
\end{aligned}
$$

Por causa da função $\delta\left(\xi+k_{1}+k_{2}\right), k_{2}=-\xi-k_{1}, \operatorname{logo}$

$$
\begin{aligned}
\delta\left(\xi+k_{1}+k_{2}\right) \sqrt{g\left(z+k_{2}\right)} & =\delta\left(\xi+k_{1}+k_{2}\right) \sqrt{g\left(z+\frac{k_{2}}{2}-\frac{\xi}{2}-\frac{k_{1}}{2}\right)} \\
& =\delta\left(\xi+k_{1}+k_{2}\right) \sqrt{g\left(x+\frac{k_{2}-k_{1}}{2}\right)} .
\end{aligned}
$$

As regras de comutação que seguem de (B.2)

$$
\begin{aligned}
& \frac{\partial}{\partial k_{2}^{\mu}} \sqrt{g\left(x+\frac{k_{2}-k_{1}}{2}\right)}=\sqrt{g\left(x+\frac{k_{2}-k_{1}}{2}\right)}\left(\frac{\partial}{\partial k_{2}^{\mu}}+\frac{1}{2} \Gamma_{\mu}\left(x+\frac{k_{2}-k_{1}}{2}\right)\right) \\
& \frac{\partial}{\partial k_{1}^{\mu}} \sqrt{g\left(x+\frac{k_{2}-k_{1}}{2}\right)}=\sqrt{g\left(x+\frac{k_{2}-k_{1}}{2}\right)}\left(\frac{\partial}{\partial k_{1}^{\mu}}-\frac{1}{2} \Gamma_{\mu}\left(x+\frac{k_{2}-k_{1}}{2}\right)\right)
\end{aligned}
$$

implicam na forma

$$
\begin{aligned}
& \int \widetilde{F}(x, \eta) \widetilde{G}(x, \zeta) \sqrt{g(w)} d w \\
& =\left.F\left(x, i \hbar \frac{\partial}{\partial k_{1}}\right) G\left(x, i \hbar \frac{\partial}{\partial k_{2}}\right) \sqrt{g\left(x+\frac{k_{2}-k_{1}}{2}\right)} \delta\left(\xi+k_{1}+k_{2}\right)\right|_{k_{1}=k_{2}=0} \\
& =\sqrt{g(x)} F\left(x, i \hbar \frac{\partial}{\partial k_{1}}-\frac{i \hbar}{2} \Gamma_{\mu}\left(x+\frac{k_{2}-k_{1}}{2}\right)\right) \times \\
& \quad \times\left. G\left(x, i \hbar \frac{\partial}{\partial k_{2}}+\frac{i \hbar}{2} \Gamma_{\mu}\left(x+\frac{k_{2}-k_{1}}{2}\right)\right) \delta\left(\xi+k_{1}+k_{2}\right)\right|_{k_{1}=k_{2}=0} .
\end{aligned}
$$

Os operadores $F\left(x, i \hbar \frac{\partial}{\partial k_{1}}-\frac{i \hbar}{2} \Gamma_{\mu}\left(x+\frac{k_{2}-k_{1}}{2}\right)\right)$ podem ser expandidos até ordem de 
$\hbar$ como

$$
\begin{aligned}
& F\left(x, i \hbar \frac{\partial}{\partial k_{1}}-\right.\left.\frac{i \hbar}{2} \Gamma_{\mu}\left(x+\frac{k_{2}-k_{1}}{2}\right)\right)= \\
& F\left(x, i \hbar \frac{\partial}{\partial k_{1}}\right)-\frac{i \hbar}{2} \Gamma_{\mu}\left(x+\frac{k_{2}-k_{1}}{2}\right) \frac{\partial F}{\partial p_{\mu}}\left(x, i \hbar \frac{\partial}{\partial k_{1}}\right)+O\left(\hbar^{2}\right) . \\
& G\left(x, i \hbar \frac{\partial}{\partial k_{2}}+\frac{i \hbar}{2} \Gamma_{\mu}\left(x+\frac{k_{2}-k_{1}}{2}\right)\right)= \\
& G\left(x, i \hbar \frac{\partial}{\partial k_{2}}\right)+\frac{i \hbar}{2} \Gamma_{\mu}\left(x+\frac{k_{2}-k_{1}}{2}\right) \frac{\partial G}{\partial p_{\mu}}\left(x, i \hbar \frac{\partial}{\partial k_{2}}\right)+O\left(\hbar^{2}\right) .
\end{aligned}
$$

$\mathrm{Assim}^{3}$,

$$
\begin{aligned}
& \int \widetilde{F}(x, \eta) \widetilde{G}(x, \zeta) \sqrt{g(w)} d w= \\
& \sqrt{g(x)}\left\{F\left(x, i \hbar \frac{\partial}{\partial k_{1}}\right) G\left(x, i \hbar \frac{\partial}{\partial k_{2}}\right)+\frac{i \hbar}{2} \Gamma_{\mu}\left(x+\frac{k_{2}-k_{1}}{2}\right) \times\right. \\
& \left.\times\left[F\left(x, i \hbar \frac{\partial}{\partial k_{1}}\right) \frac{\partial G}{\partial p_{\mu}}\left(x, i \hbar \frac{\partial}{\partial k_{2}}\right)-\frac{\partial F}{\partial p_{\mu}}\left(x, i \hbar \frac{\partial}{\partial k_{1}}\right) G\left(x, i \hbar \frac{\partial}{\partial k_{2}}\right)\right]\right\} \times \\
& \times\left.\delta\left(\xi+k_{1}+k_{2}\right)\right|_{k_{1}=k_{2}=0}+O\left(\hbar^{2}\right) \\
& =\sqrt{g(x)}\left\{F\left(x, i \hbar \frac{\partial}{\partial \xi}\right) G\left(x, i \hbar \frac{\partial}{\partial \xi}\right)+\frac{i \hbar}{2} \Gamma_{\mu}(x) \times\right. \\
& \left.\times\left[F\left(x, i \hbar \frac{\partial}{\partial \xi}\right) \frac{\partial G}{\partial p_{\mu}}\left(x, i \hbar \frac{\partial}{\partial \xi}\right)-\frac{\partial F}{\partial p_{\mu}}\left(x, i \hbar \frac{\partial}{\partial \xi}\right) G\left(x, i \hbar \frac{\partial}{\partial \xi}\right)\right]\right\} \delta(\xi)+O\left(\hbar^{2}\right) \\
& =\sqrt{g(x)} \widetilde{F G}(x, \xi)+\sqrt{g(x)} \frac{i \hbar}{2} \Gamma_{\mu}(x)\left(F \frac{\partial G}{\partial p_{\mu}}-\frac{\partial F}{\partial p_{\mu}} G\right)^{\sim}(x, \xi)+O\left(\hbar^{2}\right) \text {. }
\end{aligned}
$$

Aplicando esta fórmula à integral $I_{1}$ até a ordem de $\hbar$, temos

$$
I_{1}=\frac{1}{\sqrt{g(x)}} \widetilde{F G}(x, \xi)+\frac{i \hbar}{2 \sqrt{g(x)}} \Gamma_{\mu}(x)\left(F \frac{\partial G}{\partial p_{\mu}}-\frac{\partial F}{\partial p_{\mu}} G\right)^{\sim}(x, \xi)+O\left(\hbar^{2}\right) .
$$

\footnotetext{
${ }^{3}$ Deve-se notar que

$F\left(x, i \hbar \frac{\partial}{\partial k_{1}}\right)\left(\frac{i \hbar}{2} \Gamma_{\mu}\left(x+\frac{k_{2}-k_{1}}{2}\right)\right)=\left(\frac{i \hbar}{2} \Gamma_{\mu}\left(x+\frac{k_{2}-k_{1}}{2}\right)\right) F\left(x, i \hbar \frac{\partial}{\partial k_{1}}\right)+O\left(\hbar^{2}\right)$
} 
Precisamos aplicar (3.45) apenas até a ordem $\hbar^{0}$ às integrais $I_{2}$ e $I_{3}$ :

$$
\begin{aligned}
& I_{2}=\frac{i \hbar}{2 \sqrt{g(x)}} \widehat{\{F, G\}}(x, \xi)+O\left(\hbar^{2}\right) \\
& I_{3}=\frac{i \hbar}{2 \sqrt{g(x)}} \Gamma_{\mu}(x)\left(\frac{\partial F}{\partial p_{\mu}} G-F \frac{\partial G}{\partial p_{\mu}}\right)^{\sim}(x, \xi)+O\left(\hbar^{2}\right)
\end{aligned}
$$

Finalmente, podemos expressar a integral (3.44) como

$$
\frac{1}{\sqrt{g(x)}}\left(F *_{\mathrm{W}, \mathcal{M}} G\right)^{\sim}(x, \xi)=\frac{1}{\sqrt{g(x)}} \widetilde{F G}(x, \xi)+\frac{i \hbar}{2 \sqrt{g(x)}} \widetilde{\{F, G\}}(x, \xi)+O\left(\hbar^{2}\right)
$$

ou, eliminando as transformações de Fourier,

$$
\left(F *_{\mathrm{W}, \mathcal{M}} G\right)(x, p)=F G(x, p)+\frac{i \hbar}{2}\{F, G\}(x, p)+O\left(\hbar^{2}\right) .
$$

Esta equação mostra que o produto de símbolos $*_{\mathrm{W}, \mathcal{M}}$ obedece às duas condições (1.3) e (1.4)

$$
\begin{gathered}
\lim _{\hbar \rightarrow 0} F *_{\mathrm{W}, \mathcal{M}} G=F G \\
\lim _{\hbar \rightarrow 0} \frac{1}{i \hbar}\left(F *_{\mathrm{W}, \mathcal{M}} G-G *_{\mathrm{W}, \mathcal{M}} F\right)=\{F, G\}
\end{gathered}
$$

e, portanto, a quantização $Q^{\mathrm{W}, \mathcal{M}}$ tem limite clássico.

Pela relação (3.37), temos

$$
F^{\mathrm{W}, 1}(x, \xi)=\mathcal{F}\left(x,-i \hbar \frac{\partial}{\partial p}\right) F^{\mathrm{W}, \mathcal{F}}(x, \xi)=\mathcal{M}\left(x,-i \hbar \frac{\partial}{\partial p}\right) F^{\mathrm{W}, \mathcal{M}}(x, \xi)
$$

e, portanto,

$$
\begin{gathered}
F^{\mathrm{W}, \mathcal{F}}(x, \xi)=\mathcal{G}\left(x,-i \hbar \frac{\partial}{\partial p}\right) F^{\mathrm{W}, \mathcal{M}}(x, \xi), \\
\mathcal{G}(x, \xi)=\frac{\mathcal{M}(x, \xi)}{\mathcal{F}(x, \xi)}=1+\frac{1}{2} \mathcal{G}_{\alpha \beta}(x) \xi^{\alpha} \xi^{\beta}+O\left(\xi^{4}\right),
\end{gathered}
$$

com $\mathcal{G}_{\alpha \beta}(x)=\mathcal{G}_{\beta \alpha}(x)$. A estrutura da expansão de $\mathcal{G}(x, \xi)$ decorre do fato de que tanto $\mathcal{M}$ quanto $\mathcal{F}$ terem esta mesma estrutura. Esta relação permite expressar o produto de símbolos $*_{\mathrm{W}, \mathcal{F}}$ segundo a quantização $Q^{\mathrm{W}, \mathcal{F}}$. Sejam $F^{\mathrm{W}, \mathcal{F}}, F^{\mathrm{W}, \mathcal{M}}, G^{\mathrm{W}, \mathcal{F}}$ e $G^{\mathrm{W}, \mathcal{M}}$ os $\mathcal{F}$ - e os $\mathcal{M}$ - símbolos dos operadores $\hat{F}$ e $\hat{G}$ respectivamente, então

$$
F^{\mathrm{W}, \mathcal{F}} *_{\mathrm{W}, \mathcal{F}} G^{\mathrm{W}, \mathcal{F}}=\mathcal{G}\left(F^{\mathrm{W}, \mathcal{M}} *_{\mathrm{W}, \mathcal{M}} G^{\mathrm{W}, \mathcal{M}}\right)
$$


onde $\mathcal{G}=\mathcal{G}\left(x,-i \hbar \frac{\partial}{\partial p}\right)$. A relação inversa $F^{\mathrm{W}, \mathcal{M}}=\mathcal{G}^{-1} F^{\mathrm{W}, \mathcal{F}}$ permite então expressar $*_{\mathrm{W}, \mathcal{F}}$ em termos de $*_{\mathrm{W}, \mathcal{M}}$ como

$$
F *_{\mathrm{W}, \mathcal{F}} G=\mathcal{G}\left(\left(\mathcal{G}^{-1} F\right) *_{\mathrm{W}, \mathcal{M}}\left(\mathcal{G}^{-1} G\right)\right)
$$

Pela expansão de $\mathcal{G}(x, \xi)$, temos

$$
\begin{aligned}
F *_{\mathrm{W}, \mathcal{F}} G= & F *_{\mathrm{W}, \mathcal{M}} G-\frac{\hbar^{2}}{2}\left[\mathcal{G}_{\alpha \beta} \frac{\partial^{2}}{\partial p_{\alpha} \partial p_{\beta}}\left(F *_{\mathrm{W}, \mathcal{M}} G\right)\right. \\
& \left.-\left(\mathcal{G}_{\alpha \beta} \frac{\partial^{2} F}{\partial p_{\alpha} \partial p_{\beta}}\right) *_{\mathrm{W}, \mathcal{M}} G-F *_{\mathrm{w}, \mathcal{M}}\left(\mathcal{G}_{\alpha \beta} \frac{\partial^{2} G}{\partial p_{\alpha} \partial p_{\beta}}\right)\right]+O\left(\hbar^{4}\right) .
\end{aligned}
$$

Substituindo nesta expressão a aproximação (3.46),

$$
F *_{\mathrm{W}, \mathcal{F}} G=F *_{\mathrm{W}, \mathcal{M}} G-\hbar^{2} \mathcal{G}_{\alpha \beta} \frac{\partial F}{\partial p_{\alpha}} \frac{\partial G}{\partial p_{\beta}}+O\left(\hbar^{3}\right)
$$

Esta igualdade implica que $F *_{\mathrm{W}, \mathcal{F}} G=F *_{\mathrm{W}, \mathcal{M}} G+O\left(\hbar^{2}\right)$ e, portanto, vale também $\operatorname{para} *_{\mathrm{W}, \mathcal{F}}$

$$
\left(F *_{\mathrm{W}, \mathcal{F}} G\right)(x, p)=F G(x, p)+\frac{i \hbar}{2}\{F, G\}(x, p)+O\left(\hbar^{2}\right)
$$

Esta expansão mostra que o produto de símbolos $*_{\mathrm{W}, \mathcal{F}}$ também obedece às condições (3.47) e (3.48) do limite clássico.

A fórmula (3.36) pode ser escrita como

$$
\begin{gathered}
F^{\mathrm{W}, \mathcal{F}}(x, p)=\mathcal{O} F_{\omega, \mathcal{F}}(x, p), \\
\mathcal{O}=\Lambda_{\omega^{1}}\left(x,-i \hbar \frac{\partial}{\partial p}\right) \Omega(-\hbar \Delta) .
\end{gathered}
$$

Pela fórmula (3.21), a função $\Omega$ tem a expansão $\Omega(k)=1+\omega^{1} k+O\left(k^{2}\right)$. As fórmulas (3.18) e (3.14) implicam nas expansões

$$
\begin{aligned}
O & =1-\hbar \omega^{1}\left(\frac{\partial^{2}}{\partial x^{\mu} \partial p_{\mu}}+p_{\mu} \Gamma_{\alpha \beta}^{\mu}(x) \frac{\partial^{2}}{\partial p_{\alpha} \partial p_{\beta}}\right)+O\left(\hbar^{2}\right) \\
\mathcal{O}^{-1} & =1+\hbar \omega^{1}\left(\frac{\partial^{2}}{\partial x^{\mu} \partial p_{\mu}}+p_{\mu} \Gamma_{\alpha \beta}^{\mu}(x) \frac{\partial^{2}}{\partial p_{\alpha} \partial p_{\beta}}\right)+O\left(\hbar^{2}\right) .
\end{aligned}
$$

Podemos agora relacionar os produtos $*_{\mathrm{W}, \mathcal{F}}$ e $*_{\omega, \mathcal{F}}$ da mesma maneira que foi feito para $*_{\mathrm{W}, 1}$ e $*_{\mathrm{W}, \mathcal{F}}$ :

$$
F *_{\omega, \mathcal{F}} G=\mathcal{O}^{-1}\left((\mathcal{O} F) *_{\mathrm{W}, \mathcal{F}}(\mathcal{O} G)\right)
$$


Usando as expressões para $\mathcal{O}$ e $\mathcal{O}^{-1}$

$$
\begin{array}{r}
F *_{\omega, \mathcal{F}} G=F *_{\mathrm{W}, \mathcal{F}} G+\hbar \omega^{1}\left[\left(\frac{\partial^{2}}{\partial x^{\mu} \partial p_{\mu}}+p_{\mu} \Gamma_{\alpha \beta}^{\mu} \frac{\partial^{2}}{\partial p_{\alpha} \partial p_{\beta}}\right)\left(F *_{\mathrm{W}, \mathcal{F}} G\right)\right. \\
\left.-\left(\frac{\partial^{2} F}{\partial x^{\mu} \partial p_{\mu}}+p_{\mu} \Gamma_{\alpha \beta}^{\mu} \frac{\partial^{2} F}{\partial p_{\alpha} \partial p_{\beta}}\right) *_{\mathrm{W}, \mathcal{F}} G+F *_{\mathrm{W}, \mathcal{F}}\left(\frac{\partial^{2} G}{\partial x^{\mu} \partial p_{\mu}}+p_{\mu} \Gamma_{\alpha \beta}^{\mu} \frac{\partial^{2} G}{\partial p_{\alpha} \partial p_{\beta}}\right)\right] \\
+O\left(\hbar^{2}\right)
\end{array}
$$

Dentro dos colchetes, podemos substituir as aproximações $F *_{\mathrm{W}, \mathcal{F}} G=F G+O(\hbar)$,

$$
\begin{aligned}
F *_{\omega, \mathcal{F}} G= & F G+\frac{i \hbar}{2}\{F, G\}+\hbar \omega^{1}\left[\left(\frac{\partial^{2}}{\partial x^{\mu} \partial p_{\mu}}+p_{\mu} \Gamma_{\alpha \beta}^{\mu} \frac{\partial^{2}}{\partial p_{\alpha} \partial p_{\beta}}\right)(F G)\right. \\
& \left.-\left(\frac{\partial^{2} F}{\partial x^{\mu} \partial p_{\mu}}+p_{\mu} \Gamma_{\alpha \beta}^{\mu} \frac{\partial^{2} F}{\partial p_{\alpha} \partial p_{\beta}}\right) G+F\left(\frac{\partial^{2} G}{\partial x^{\mu} \partial p_{\mu}}+p_{\mu} \Gamma_{\alpha \beta}^{\mu} \frac{\partial^{2} G}{\partial p_{\alpha} \partial p_{\beta}}\right)\right] .
\end{aligned}
$$

Simplificando as derivadas, obtemos finalmente

$$
\begin{aligned}
F *_{\omega, \mathcal{F}} G= & F G+\frac{i \hbar}{2}\{F, G\} \\
& +\hbar \omega^{1}\left[\frac{\partial F}{\partial x^{\mu}} \frac{\partial G}{\partial p_{\mu}}+\frac{\partial F}{\partial p_{\mu}} \frac{\partial G}{\partial x^{\mu}}+p_{\mu} \Gamma_{\alpha \beta}^{\mu}\left(\frac{\partial F}{\partial p_{\alpha}} \frac{\partial G}{\partial p_{\beta}}+\frac{\partial F}{\partial p_{\beta}} \frac{\partial G}{\partial p_{\alpha}}\right)\right] \\
& +O\left(\hbar^{2}\right) .
\end{aligned}
$$

A quantidade entre colchetes é simétrica pela permutação de $F$ e $G$. Assim, o produto geral $*_{\omega, \mathcal{F}}$ obedece às condições (1.3) e (1.4), ou seja, a quantização geral $Q_{\omega, \mathcal{F}}$ tem limite clássico.

\subsection{Quantização da partícula livre em um espaço curvo}

A partícula livre em um espaço curvo com métrica $g_{\mu \nu}(x)$ tem hamiltoniana

$$
H(x, p)=\frac{1}{2 m} g^{\mu \nu}(x) p_{\mu} p_{\nu}
$$

O resultado (2.75) depende da expressão (2.71), que vale para a quantização $Q_{\omega, 1}$ como foi demonstrado na seção 3.1. Assim, na representação $\psi(x)=\left\langle\left. x\right|_{\omega^{1}} \psi\right\rangle$ para o espaço de Hilbert $\mathcal{R}$,

$$
\left(Q_{\omega, 1}(H)\right)_{x}=-\frac{\hbar^{2}}{2 m} \frac{1}{\sqrt{g}} \frac{\partial}{\partial x^{\mu}} \sqrt{g} g^{\mu \nu} \frac{\partial}{\partial x^{\nu}} .
$$


A quantização segundo $Q_{\omega, \mathcal{F}}$ pode ser calculada através da fórmula (3.38). Precisamos calcular a ação do operador $\Delta(3.18)$ sobre $H$ :

$$
\begin{aligned}
\Delta H & =\frac{1}{2 m}\left(\frac{\partial^{2}}{\partial x^{\mu} \partial p_{\mu}}+\Gamma_{\alpha} \frac{\partial}{\partial p_{\alpha}}+p_{\mu} \Gamma_{\alpha \beta}^{\mu} \frac{\partial^{2}}{\partial p_{\alpha} \partial p_{\beta}}\right) g^{\rho \sigma} p_{\rho} p_{\sigma} \\
& =\frac{p_{\rho}}{m}\left(\frac{\partial g^{\rho \mu}}{\partial x^{\mu}}+g^{\rho \sigma} \Gamma_{\sigma}+\Gamma_{\alpha \beta}^{\rho} g^{\alpha \beta}\right)=0
\end{aligned}
$$

Pode ser verificado que a expressão entre parênteses acima é zero usando a expressão (B.1) para a conexão de Levi-Civita. Assim,

$$
\Omega(-\hbar \Delta) H=\Omega(0) H=H .
$$

A expansão $\mathcal{F}(x, \xi)=1+\frac{1}{2} \mathcal{F}_{\alpha \beta}(x) \xi^{\alpha} \xi^{\beta}+O\left(\xi^{4}\right)$ postulada na seção 3.6 implica que

$$
\begin{aligned}
\mathcal{F}\left(x,-i \hbar \frac{\partial}{\partial p}\right) H & =\frac{1}{2 m}\left(1-\frac{\hbar^{2}}{2} \mathcal{F}_{\alpha \beta}(x) \frac{\partial^{2}}{\partial p_{\alpha} \partial p_{\beta}}\right) g^{\mu \nu}(x) p_{\mu} p_{\nu} \\
& =\frac{1}{2 m} g^{\mu \nu}(x) p_{\mu} p_{\nu}-\frac{\hbar^{2}}{2 m} \mathcal{F}_{\alpha \beta}(x) g^{\alpha \beta}(x) \\
& =\frac{1}{2 m} g^{\mu \nu}(x) p_{\mu} p_{\nu}+\frac{\hbar^{2}}{6 m} R(x),
\end{aligned}
$$

onde $R(x)$ é o escalar de curvatura.

Observando que $\Delta\left(\mathcal{F}_{\alpha \beta}(x) g^{\alpha \beta}(x)\right)=0$, pois $\mathcal{F}_{\alpha \beta}(x) g^{\alpha \beta}(x)$ não depende de $p$, podemos calcular $Q_{\omega, \mathcal{F}}(F)$ através de (3.38):

$$
\begin{aligned}
Q_{\omega, \mathcal{F}}(H) & =Q_{\omega, 1}\left([\Omega(-\hbar \Delta)]^{-1} \mathcal{F}\left(x,-i \hbar \frac{\partial}{\partial p}\right) \Omega(-\hbar \Delta) H\right) \\
& =Q_{\omega, 1}\left([\Omega(-\hbar \Delta)]^{-1} \mathcal{F}\left(x,-i \hbar \frac{\partial}{\partial p}\right) H\right) \\
& =Q_{\omega, 1}\left([\Omega(-\hbar \Delta)]^{-1}\left(\frac{1}{2 m} g^{\mu \nu}(x) p_{\mu} p_{\nu}-\frac{\hbar^{2}}{2 m} \mathcal{F}_{\alpha \beta}(x) g^{\alpha \beta}(x)\right)\right) \\
& =Q_{\omega, 1}\left(\frac{1}{2 m} g^{\mu \nu}(x) p_{\mu} p_{\nu}-\frac{\hbar^{2}}{2 m} \mathcal{F}_{\alpha \beta}(x) g^{\alpha \beta}(x)\right)
\end{aligned}
$$

Levando em conta a propriedade $Q_{\omega, 1}(F(x))=F(\hat{x})$,

$$
\left(Q_{\omega, \mathcal{F}}(H)\right)_{x}=-\frac{\hbar^{2}}{2 m} \frac{1}{\sqrt{g}} \frac{\partial}{\partial x^{\mu}} \sqrt{g} g^{\mu \nu} \frac{\partial}{\partial x^{\nu}}-\frac{\hbar^{2}}{2 m} \mathcal{F}_{\alpha \beta}(x) g^{\alpha \beta}(x)
$$

Se escolhermos $\mathcal{F}=\mathcal{M}$, pela expansão (3.32), obtemos a forma tradicional do 
potencial quântico

$$
\left(Q_{\omega, \mathcal{F}}(H)\right)_{x}=-\frac{\hbar^{2}}{2 m} \frac{1}{\sqrt{g}} \frac{\partial}{\partial x^{\mu}} \sqrt{g} g^{\mu \nu} \frac{\partial}{\partial x^{\nu}}+\frac{\hbar^{2}}{6 m} R(x)
$$

onde $R(x)=R_{\alpha \beta}(x) g^{\alpha \beta}(x)$ é o escalar de curvatura. No entanto, funções $\mathcal{F}$ podem ser escolhidas para potenciais quânticos mais gerais. Por exemplo, escolhendo uma função $f(R)$ tal que $\lim _{R \rightarrow 0} f(R) / R<\infty$ e arbitrária em outros aspectos, podemos definir

$$
\mathcal{F}(x, \xi)=1+\frac{f(R(x))}{R(x)} R_{\alpha \beta}(x)+\cdots
$$

Para esta função, temos

$$
\left(Q_{\omega, \mathcal{F}}(H)\right)_{x}=-\frac{\hbar^{2}}{2 m} \frac{1}{\sqrt{g}} \frac{\partial}{\partial x^{\mu}} \sqrt{g} g^{\mu \nu} \frac{\partial}{\partial x^{\nu}}-\frac{\hbar^{2}}{m} f(R(x)) .
$$




\section{Apêndice A}

\section{Propriedades do operador $e^{i(\eta \hat{x}+\hat{p} \xi)}$}

Para qualquer número complexo $\alpha$, temos que o comutador $[i(\eta \hat{x}+\alpha \hat{p} \xi), i(1-\alpha) \hat{p} \xi]=$ $-i \hbar(1-\alpha) \eta \xi$ é um número e, neste caso particular, a fórmula de Baker-CampbellHausdorff se expressa como $e^{\hat{A}+\hat{B}}=e^{-\frac{1}{2}[\hat{A}, \hat{B}]} e^{\hat{A}} e^{\hat{B}}$, permitindo separar a exponencial de operadores $\hat{x}^{\mu}$ e $\hat{p}_{\mu}$ :

$$
\begin{aligned}
e^{i(\eta \hat{x}+\hat{p} \xi)} & =e^{i(\eta \hat{x}+\alpha \hat{p} \xi+(1-\alpha) \hat{p} \xi)} \\
& =e^{i \hbar \frac{1-\alpha}{2} \eta \xi} e^{i(\eta \hat{x}+\alpha \hat{p} \xi)} e^{i(1-\alpha) \hat{p} \xi}
\end{aligned}
$$

Usando a relação de comutação $[i \alpha \hat{p} \xi, i \eta \hat{x}]=i \hbar \alpha \eta \xi$, temos

$$
e^{i(\eta \hat{x}+\alpha \hat{p} \xi)}=e^{-i \hbar \frac{\alpha}{2} \eta \xi} e^{i \alpha \hat{p} \xi} e^{i \eta \hat{x}}
$$

e portanto

$$
e^{i(\eta \hat{x}+\hat{p} \xi)}=e^{-i \hbar\left(\alpha-\frac{1}{2}\right) \eta \xi} e^{i \alpha \hat{p} \xi} e^{i \eta \hat{x}} e^{i(1-\alpha) \hat{p} \xi}
$$

Tomando $\alpha=1$, temos

$$
e^{i(\eta \hat{x}+\hat{p} \xi)}=e^{-\frac{i \hbar}{2} \eta \xi} e^{i \hat{p} \xi} e^{i \eta \hat{x}}
$$

o que permite calcular o elemento de matriz

$$
\begin{aligned}
\left\langle x\left|e^{i(\eta \hat{x}+\hat{p} \xi)}\right| y\right\rangle & =e^{i \eta\left(y-\frac{\hbar}{2} \xi\right)}\left\langle x\left|e^{i \hat{p} \xi}\right| y\right\rangle \\
& =e^{i \eta\left(y-\frac{\hbar}{2} \xi\right)} \delta(x+\hbar \xi-y) \\
& =e^{i \eta \frac{x+y}{2}} \delta(x+\hbar \xi-y) \\
& =\hbar^{-D} e^{i \eta \frac{x+y}{2}} \delta\left(\xi-\frac{y-x}{\hbar}\right)
\end{aligned}
$$

segundo o conjunto completo $\{|x\rangle\}$ padrão, normalizado como $\langle x \mid y\rangle=\delta(x-y)$ tal 
que $\left\langle x\left|\hat{p}_{\mu}\right| y\right\rangle=-i \hbar \frac{\partial}{\partial x^{\mu}} \delta(x-y)$. Dado um operador $\hat{A}$ em $\mathcal{R}$, podemos definir os coeficientes

$$
c(\eta, \xi)=\left(\frac{\hbar}{2 \pi}\right)^{D} \int\left\langle x-\frac{\hbar}{2} \xi|\hat{A}| x+\frac{\hbar}{2} \xi\right\rangle e^{-i \eta x} d x .
$$

Podemos então demonstrar que

$$
\hat{A}=\int c(\eta, \xi) e^{i(\eta \hat{x}+\hat{p} \xi)} d \eta d \xi
$$

tomando o elemento de matriz do segundo membro:

$$
\begin{aligned}
\left\langle x\left|\int c(\eta, \xi) e^{i(\eta \hat{x}+\hat{p} \xi)} d \eta d \xi\right| y\right\rangle & =\hbar^{-D} \int c(\eta, \xi) e^{i \eta \frac{x+y}{2}} \delta\left(\xi-\frac{y-x}{\hbar}\right) d \eta d \xi \\
& =\hbar^{-D} \int c\left(\eta, \frac{y-x}{\hbar}\right) e^{i \eta \frac{x+y}{2}} d \eta \\
& =\left(\frac{1}{2 \pi}\right)^{D} \int\left\langle z-\frac{1}{2}(y-x)|\hat{A}| z+\frac{1}{2}(y-x)\right\rangle e^{i \eta\left(\frac{x+y}{2}-z\right)} d z d \eta \\
& =\int\left\langle z-\frac{1}{2}(y-x)|\hat{A}| z+\frac{1}{2}(y-x)\right\rangle \delta\left(\frac{x+y}{2}-z\right) d z \\
& =\langle x|\hat{A}| y\rangle .
\end{aligned}
$$

Podemos também calcular as relações de comutação de $\hat{x}^{\mu}$ e $\hat{p}_{\mu} \operatorname{com} e^{i(\eta \hat{x}+\hat{p} \xi)}$ usando (A.2):

$$
\begin{aligned}
{\left[\hat{x}^{\mu}, e^{i(\eta \hat{x}+\hat{p} \xi)}\right]=e^{-\frac{i \hbar}{2} \eta \xi}\left[\hat{x}^{\mu}, e^{i \hat{p} \xi}\right] e^{i \eta \hat{x}}=-\hbar \xi^{\mu} e^{i(\eta \hat{x}+\hat{p} \xi)} } \\
{\left[\hat{p}_{\mu}, e^{i(\eta \hat{x}+\hat{p} \xi)}\right]=e^{-\frac{i \hbar}{2} \eta \xi} e^{i \hat{p} \xi}\left[\hat{p}_{\mu}, e^{i \eta \hat{x}}\right]=\hbar \eta_{\mu} e^{i(\eta \hat{x}+\hat{p} \xi)} }
\end{aligned}
$$




\section{Apêndice B}

\section{Definições e fórmulas da geometria riemanniana}

Consideremos um espaço de riemanniano (ou pseudo riemanniano) em $D$ dimensões com coordenadas locais $x$ e tensor métrico $g_{\mu \nu}(x), g_{\mu \nu}(x)=g_{\nu \mu}(x)$. O inverso do tensor métrico $g^{\mu \nu}(x)$, tal que

$$
g^{\mu \nu}(x) g_{\nu \rho}(x)=\delta_{\rho}^{\mu}
$$

é um tensor contravariante. A conexão de Levi-Civita é dada por

$$
\Gamma_{\alpha \beta}^{\mu}(x)=\frac{1}{2} g^{\mu \rho}\left(\frac{\partial g_{\rho \alpha}}{\partial x^{\beta}}+\frac{\partial g_{\rho \beta}}{\partial x^{\alpha}}-\frac{\partial g_{\alpha \beta}}{\partial x^{\rho}}\right)
$$

A contração $\Gamma_{\alpha \mu}^{\mu}(x) \equiv \Gamma_{\alpha}(x)$ tem a propriedade de ser um gradiente:

$$
\Gamma_{\alpha}(x)=\frac{1}{\sqrt{g}} \frac{\partial \sqrt{g}}{\partial x^{\alpha}}=\frac{\partial \ln (\sqrt{g})}{\partial x^{\alpha}},
$$

onde

$$
g(x)=\left|\operatorname{det}\left(g_{\mu \nu}(x)\right)\right| .
$$

(A maior parte dos livros define $g(x)=\operatorname{det}\left(g_{\mu \nu}(x)\right)$. Nós nunca precisaremos do sinal de $\operatorname{det}\left(g_{\mu \nu}(x)\right)$.) Sejam

$$
x^{\prime \mu}=\varphi^{\mu}(x)
$$

novas coordenadas, onde $\varphi: \mathbb{R}^{D} \rightarrow \mathbb{R}^{D}$ é um difeomorfismo. Como é usual, denotamos as derivadas parciais da função $\varphi(x)$ e da função inversa $\varphi^{-1}\left(x^{\prime}\right)$ como $\frac{\partial x^{\prime \alpha}}{\partial x^{\mu}}(x)=\frac{\partial \varphi^{\alpha}}{\partial x^{\mu}}(x)$ e $\frac{\partial x^{\mu}}{\partial x^{\prime \alpha}}\left(x^{\prime}\right)=\frac{\partial\left(\varphi^{-1}\right)^{\mu}}{\partial x^{\prime \alpha}}\left(x^{\prime}\right)$. Num abuso de linguagem, podemos também expressar $\frac{\partial x^{\prime \alpha}}{\partial x^{\mu}}$ como função de $x^{\prime}$ através de $\frac{\partial x^{\prime \alpha}}{\partial x^{\mu}}\left(x^{\prime}\right)=\frac{\partial \varphi^{\alpha}}{\partial x^{\mu}}\left(\varphi^{-1}\left(x^{\prime}\right)\right)$ e, da 
mesma forma, $\frac{\partial x^{\mu}}{\partial x^{\prime \alpha}}(x)=\frac{\partial\left(\varphi^{-1}\right)^{\mu}}{\partial x^{\prime \alpha}}(\varphi(x))$. Nestas coordenadas, o tensor métrico se expressa como

$$
g_{\alpha \beta}^{\prime}=g_{\mu \nu} \frac{\partial x^{\mu}}{\partial x^{\prime \alpha}} \frac{\partial x^{\nu}}{\partial x^{\prime \beta}} .
$$

Tomando o determinante e a raiz quadrada em ambos os lados,

$$
\sqrt{g^{\prime}}=\sqrt{g}\left|\operatorname{det}\left(\frac{\partial x}{\partial x^{\prime}}\right)\right|
$$

Por esta fórmula, vemos que o elemento de volume riemanniano $\sqrt{g(x)} d x$ é invariante, isto é:

$$
\sqrt{g} d x=\sqrt{g}\left|\operatorname{det}\left(\frac{\partial x}{\partial x^{\prime}}\right)\right| d x^{\prime}=\sqrt{g^{\prime}} d x^{\prime}
$$

A conexão de Levi-Civita tem a lei de transformação não tensorial

$$
\Gamma_{\alpha \beta}^{\prime \mu}=\Gamma_{\gamma \delta}^{\nu} \frac{\partial x^{\prime \mu}}{\partial x^{\nu}} \frac{\partial x^{\gamma}}{\partial x^{\prime \alpha}} \frac{\partial x^{\delta}}{\partial x^{\prime \beta}}+\frac{\partial^{2} x^{\nu}}{\partial x^{\prime \alpha} \partial x^{\prime \beta}} \frac{\partial x^{\prime \mu}}{\partial x^{\nu}}
$$

O critério para a existência de um sistema de coordenadas retilíneo, isto é, o critério para que o espaço seja plano, é que o tensor de curvatura de RiemannChristoffel

$$
R_{\mu \beta \nu}^{\alpha}=\Gamma_{\mu \nu, \beta}^{\alpha}-\Gamma_{\mu \nu, \beta}^{\alpha}+\Gamma_{\rho \beta}^{\alpha} \Gamma_{\mu \nu}^{\rho}-\Gamma_{\rho \nu}^{\alpha} \Gamma_{\mu \beta}^{\rho}
$$

seja nulo. Com este tensor, podemos definir o tensor de Ricci

$$
R_{\mu \nu}=R_{\mu \alpha \nu}^{\alpha}=\Gamma_{\mu \nu, \rho}^{\rho}-\Gamma_{\mu, \nu}+\Gamma_{\rho} \Gamma_{\mu \nu}^{\rho}-\Gamma_{\rho \nu}^{\alpha} \Gamma_{\mu \alpha}^{\rho}
$$

e o escalar de curvatura

$$
R=g^{\mu \nu} R_{\mu \nu}
$$

Se o espaço de Riemann é plano e $x$ são coordenadas ortonormais, isto é, $g_{\mu \nu}=$ $\operatorname{diag}(1,1, \ldots, 1,-1,-1, \ldots,-1)$ constante, temos $\Gamma_{\alpha \beta}^{\mu}=0$. Em novas coordenadas gerais $x^{\prime}$, teremos o elemento de volume

$$
d x=\sqrt{g^{\prime}} d x^{\prime}
$$

onde $d x=d x^{1} d x^{2} \ldots d x^{D}$ e $d x^{\prime}=d x^{\prime 1} d x^{\prime 2} \ldots d x^{\prime D}$, e a conexão de Levi-Civita

$$
\Gamma_{\alpha \beta}^{\prime \mu}=\frac{\partial^{2} x^{\nu}}{\partial x^{\prime \alpha} \partial x^{\prime \beta}} \frac{\partial x^{\prime \mu}}{\partial x^{\nu}}
$$

A conexão de Levi-Civita permite definir as derivadas covariantes:

1. de um escalar: $\nabla_{\mu} F(x)=\frac{\partial F}{\partial x^{\mu}}$ 
2. de um vetor ou tensor:

$$
\begin{aligned}
\nabla_{\mu} A^{\nu} & =\frac{\partial A^{\nu}}{\partial x^{\mu}}+\Gamma_{\mu \rho}^{\nu} A^{\rho}=\left(\delta_{\rho}^{\nu} \frac{\partial}{\partial x^{\mu}}+\Gamma_{\mu \rho}^{\nu}\right) A^{\rho} \\
\nabla_{\mu} A_{\nu} & =\frac{\partial A_{\nu}}{\partial x^{\mu}}-\Gamma_{\mu \nu}^{\rho} A_{\rho}=\left(\delta_{\nu}^{\rho} \frac{\partial}{\partial x^{\mu}}-\Gamma_{\mu \nu}^{\rho}\right) A_{\rho} \\
\nabla_{\mu} T_{\alpha \beta} & =\frac{\partial T_{\alpha \beta}}{\partial x^{\mu}}-\Gamma_{\mu \alpha}^{\rho} T_{\rho \beta}-\Gamma_{\mu \beta}^{\rho} T_{\alpha \rho}=\left(\delta_{\alpha}^{\rho} \delta_{\beta}^{\sigma} \frac{\partial}{\partial x^{\mu}}-\delta_{\beta}^{\sigma} \Gamma_{\mu \alpha}^{\rho}-\delta_{\alpha}^{\rho} \Gamma_{\mu \beta}^{\sigma}\right) T_{\rho \sigma}, \text { etc... }
\end{aligned}
$$

A derivada covariante de qualquer tensor é um tensor, incluindo os índices da própria derivada. A derivada covariante do tensor métrico é sempre nula:

$$
\nabla_{\mu} g_{\alpha \beta}=\nabla_{\mu} g^{\alpha \beta}=0
$$

A derivada covariante obecede à regra de Leibnitz para a derivada de um produto de tensores:

$$
\nabla_{\mu}\left(S_{\ldots}^{\cdots} T_{\ldots}^{\cdots}\right)=\left(\nabla_{\mu} S_{\ldots}^{\cdots}\right) T_{\ldots}^{\cdots}+S_{\ldots}^{\cdots}\left(\nabla_{\mu} T_{\ldots}^{\cdots}\right)
$$

A fórmula (B.2) permite expressar o divergente covariante de um vetor como

$$
\nabla_{\mu} A^{\mu}=\frac{1}{\sqrt{g}} \frac{\partial}{\partial x^{\mu}}\left(\sqrt{g} A^{\mu}\right) .
$$

Por esta fórmula, vemos que é verdadeiro o teorema de Gauss quando se usa o elemento de volume invariante na integração:

$$
\int_{R} \nabla_{\mu} A^{\mu} \sqrt{g} d x=\int_{\partial R} A^{\mu} n_{\mu} \sqrt{g} d S
$$

onde $\partial R$ é a borda do volume de integração $R, n^{\mu}$ é ortogonal a $\partial R$ e $d S$ é o elemento de área em $\partial R$. 


\section{Apêndice $\mathrm{C}$}

\section{A função exponencial}

\section{C.1 Definição e propriedades da função exponen- cial}

Desenvolveremos aqui algumas propriedades da função exponencial num espaço de Riemann. Em coordenadas locais, as curvas $x^{\mu}(\tau)=C^{\mu}(\tau)$ que obedecem à equação

$$
\frac{d^{2} C^{\mu}}{d \tau^{2}}=-\Gamma_{\alpha \beta}^{\mu}(C(\tau)) \frac{d C^{\alpha}}{d \tau} \frac{d C^{\beta}}{d \tau}
$$

chamam-se geodésicas, onde $\Gamma_{\alpha \beta}^{\mu}$ é a conexão de Levi-Civita (B.1). Elas são os mínimos da ação

$$
S[C]=\int \sqrt{g_{\mu \nu}(x) \dot{C}^{\mu} \dot{C}^{\nu}} d \tau
$$

submetidos à condição (de calibre) de velocidade constante

$$
\frac{d}{d \tau}\left[\sqrt{g_{\mu \nu}(C(\tau)) \dot{C}^{\mu}(\tau) \dot{C}^{\nu}(\tau)}\right]=0
$$

Dados um ponto de coordenadas $x^{\mu}$ e um vetor, neste ponto, de coordenadas $\xi^{\mu}$, as condições iniciais

$$
\left\{\begin{array}{l}
C^{\mu}(0)=x^{\mu} \\
\frac{d C^{\mu}}{d \tau}(0)=\xi^{\mu}
\end{array}\right.
$$

definem as funções

$$
\begin{aligned}
& \gamma(x, \xi)=C(1) \\
& \beta(x, \xi)=\dot{C}(1) .
\end{aligned}
$$




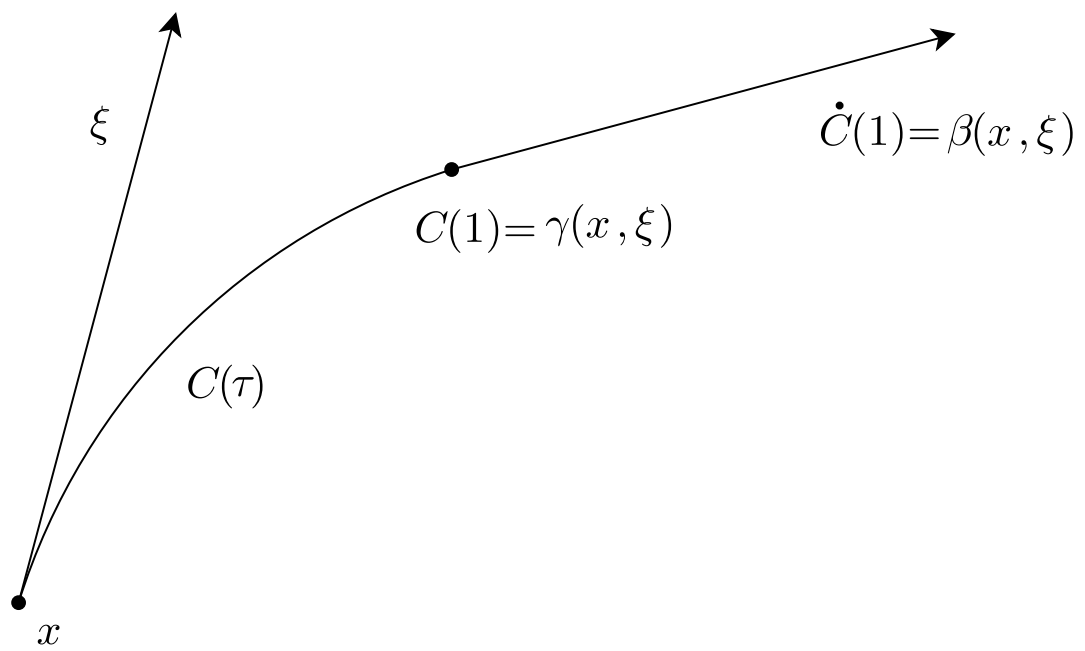

Figura C.1: Função exponencial

A função $\gamma$ é conhecida como função exponencial. Geometricamente, o ponto $\gamma(x, \xi)$ é a translação do ponto $x$ sobre uma geodésica de comprimento $\sqrt{g_{\mu \nu}(x) \xi^{\mu} \xi^{\nu}}$ inicialmente tangente a $\xi$, e $\beta(x, \xi)$ é o transporte paralelo de $\xi$ ao longo desta geodésica, no ponto final $\gamma(x, \xi)$ (ver a figura C.1). A condição (C.3) implica que $\dot{C}(1)=\beta(x, \xi)$ e $\dot{C}(0)=\xi$ têm o mesmo tamanho, ou seja,

$$
g_{\mu \nu}(x) \xi^{\mu} \xi^{\nu}=g_{\mu \nu}(\gamma(x, \xi)) \beta^{\mu}(x, \xi) \beta^{\nu}(x, \xi)
$$

A função constante $C(\tau)=x$ é solução da equação (C.1). Esta solução tem condições iniciais $C(0)=x, \dot{C}(0)=0$, portanto

$$
\gamma(x, 0)=x
$$

Seja $C(\tau)$ uma solução de (C.1) com as condições iniciais (C.4). Pode-se verificar, por substituição direta, que $K(\tau)=C(\lambda \tau)$ é também solução de (C.1) com condições iniciais $K(0)=x$ e $\dot{K}(0)=\lambda \xi$. Assim, podemos recuperar a função $C(\tau)$ através da função $\gamma(x, \xi)$ :

$$
C(\lambda)=K(1)=\gamma(x, \lambda \xi)
$$

Como conseqüência, vale a relação entre $\gamma(x, \xi)$ e $\beta(x, \xi)$

$$
\beta^{\mu}(x, \xi)=\left.\frac{d}{d \tau} \gamma^{\mu}(x, \tau \xi)\right|_{\tau=1}=\frac{\partial \gamma^{\mu}}{\partial \xi^{\alpha}}(x, \xi) \xi^{\alpha}
$$

Consideremos a equação diferencial (C.1) no espaço de fase não canônico $(x, \xi) \in$ 
$\mathbb{R}^{2 D}$. Chamamos de fluxo geodésico a propagação $(C(\tau), \dot{C}(\tau))=T_{\tau}(x, \xi)$ das condições iniciais (C.4). As coordenadas do fluxo geodésico obedecem a uma equação diferencial de primeira ordem:

$$
\begin{aligned}
T_{\tau}(x, \xi) & =\left(x_{\tau}(x, \xi), \xi_{\tau}(x, \xi)\right) \\
\frac{d}{d \tau} x_{\tau}^{\mu} & =\xi_{\tau}^{\mu} \\
\frac{d}{d \tau} \xi_{\tau}^{\mu} & =-\Gamma_{\alpha \beta}^{\mu}\left(x_{\tau}\right) \xi_{\tau}^{\alpha} \xi_{\tau}^{\beta}
\end{aligned}
$$

A equação (C.9) dá a forma explícita de $x_{\tau}(x, \xi)=\gamma(x, \tau \xi)$. Pelas equações (C.11) e (C.10), obtemos $\xi_{\tau}^{\mu}(x, \xi)=\frac{1}{\tau} \beta(x, \tau \xi)$. Assim, o fluxo geodésico $T_{\tau}: \mathbb{R}^{2 D} \rightarrow \mathbb{R}^{2 D}$ se expressa como

$$
T_{\tau}(x, \xi)=\left(\gamma(x, \tau \xi), \frac{1}{\tau} \beta(x, \tau \xi)\right) .
$$

O fluxo geodésico, como o fluxo de qualquer equação diferencial, é um difeomorfismo que obedece às propriedades de grupo comutativo

$$
\begin{aligned}
T_{\tau}\left(T_{\lambda}(x, \xi)\right) & =T_{\tau+\lambda}(x, \xi) \\
T_{\tau}^{-1} & =T_{-\tau},
\end{aligned}
$$

onde $T_{\tau}^{-1}(x, \xi)$ é a função inversa de $T_{\tau}(x, \xi)$. Fazendo $\lambda=1$ em (C.14) e tomando apenas a componente de posição, obtemos a lei de composição entre $\gamma$ e $\beta$

$$
\gamma(x,(1+\tau) \xi)=\gamma(\gamma(x, \xi), \tau \beta(x, \xi)) \text {. }
$$

As equações (C.2) e (C.3), conjuntamente equivalentes a (C.1), são covariantes sob uma transformação geral $x^{\prime}=\varphi(x)$, onde $\varphi$ é um difeomorfismo qualquer. Assim, se $C(\tau)$ é solução de (C.1) em coordenadas $x$, com condições iniciais (C.4), então $C^{\prime}(\tau)=\varphi(C(\tau))$ é solução de (C.1) nas novas coordenadas $x^{\prime}$, isto é, usando $\Gamma_{\alpha \beta}^{\prime \mu}$ (B.5), com condições iniciais $C^{\prime \mu}(0)=x^{\prime \mu}=\varphi^{\mu}(x)$ e $\dot{C}^{\prime \mu}(0)=\xi^{\prime \mu}=\frac{\partial x^{\prime \mu}}{\partial x^{\alpha}}(x) \xi^{\alpha}$. Portanto, a função exponencial $\gamma^{\prime}\left(x^{\prime}, \xi^{\prime}\right)$ definida no referencial $x^{\prime}$ por meio da conexão $\Gamma_{\alpha \beta}^{\prime \mu}$ se relacionará com $\gamma(x, \xi)$ por $\gamma^{\prime}\left(x^{\prime}, \xi^{\prime}\right)=C^{\prime}(1)=\varphi(C(1))=\varphi(\gamma(x, \xi))$, ou seja,

$$
\varphi(\gamma(x, \xi))=\gamma^{\prime}\left(\varphi(x), \frac{\partial x^{\prime}}{\partial x}(x) \xi\right)
$$

As velocidades da geodésica nas coordenadas $x$ e $x^{\prime}$ se relacionam por

$$
\dot{C}^{\prime \mu}(\tau)=\frac{\partial \varphi^{\mu}}{\partial x^{\alpha}}(C(\tau)) \frac{d C^{\alpha}}{\partial \tau}(\tau)
$$


Calculando estas derivadas em $\tau=1$, temos

$$
\beta^{\prime \mu}\left(x^{\prime}, \xi^{\prime}\right)=\beta^{\prime \mu}\left(\varphi(x), \frac{\partial x^{\prime}}{\partial x}(x) \xi\right)=\frac{\partial x^{\prime \mu}}{\partial x^{\alpha}}(\gamma(x, \xi)) \beta^{\alpha}(x, \xi)
$$

$\beta^{\alpha}(x, \xi)$ não é um campo vetorial, pois os coeficientes de transformação $\frac{\partial x^{\prime \mu}}{\partial x^{\alpha}}$ são calculados no ponto $\gamma(x, \xi)$, e não em $x$. As leis de transformação (C.17) e (C.18) mostram que o fluxo geodésico (C.13) $T_{\tau}(x, \xi)=\left(x_{\tau}(x, \xi), \xi_{\tau}(x, \xi)\right)$ se transforma como

$$
\left(x_{\tau}^{\prime}, \xi_{\tau}^{\prime}\right)=\left(\varphi\left(x_{\tau}\right), \frac{\partial x^{\prime}}{\partial x}\left(x_{\tau}\right) \xi_{\tau}\right)
$$

Notemos que se o espaço é plano e as coordenadas $x$ são euclidianas (ou pseudoeuclidianas), $\Gamma_{\alpha \beta}^{\mu}=0$ e portanto as equações (C.1) têm a forma $\frac{d^{2} C^{\mu}}{d \tau^{2}}=0$. As soluções desta equação com condições iniciais (C.4) são $C(\tau)=x+\tau \xi$, e portanto $\gamma(x, \xi)=x+\xi$ e $\beta(x, \xi)=\xi$. A função exponencial em coordenadas $x^{\prime}$ quaisquer é dada então por

$$
\varphi(x+\xi)=\gamma^{\prime}\left(\varphi(x), \frac{\partial x^{\prime}}{\partial x}(x) \xi\right)
$$

É simples obter a função $\gamma(x, \xi)$ como uma série de potências de $\xi$. De fato, podese obter a série de potências de $F(\gamma(x, \xi)$ ), para qualquer função (infinitamente diferenciável) $F(x)$. Pela regra da cadeia,

$$
\frac{d}{d \tau} F(C(\tau))=\dot{C}^{\mu}(\tau) \partial_{\mu} F(C(\tau))
$$

A derivada segunda pode ser calculada usando a equação diferencial (C.1) para reduzir $\ddot{C}$ a $\dot{C}$ :

$$
\begin{aligned}
\frac{d^{2}}{d \tau^{2}} F(C) & =\ddot{C}^{\mu} \partial_{\mu} F(C)+\dot{C}^{\mu} \dot{C}^{\nu} \partial_{\mu} \partial_{\nu} F(C) \\
& =\dot{C}^{\mu} \dot{C}^{\nu} \nabla_{\mu} \nabla_{\nu} F(C)
\end{aligned}
$$

onde $\nabla_{\mu}$ é a derivada covariante, $\nabla_{\mu} \nabla_{\nu} F=\left(\delta_{\nu}^{\rho} \partial_{\mu}-\Gamma_{\mu \nu}^{\rho}\right) \partial_{\rho} F$. É simples então verificar por indução sobre $n$ que

$$
\frac{d^{n}}{d \tau^{n}} F(C)=\dot{C}^{\mu_{1}} \ldots \dot{C}^{\mu_{n}} \nabla_{\mu_{1}} \ldots \nabla_{\mu_{n}} F(C)
$$


Usando a condição inicial (C.4),

$$
\begin{aligned}
\left.\frac{d^{n}}{d \tau^{n}} F(C(\tau))\right|_{\tau=0} & =\xi^{\mu_{1}} \ldots \xi^{\mu_{n}} \nabla_{\mu_{1}} \ldots \nabla_{\mu_{n}} F(x) \\
& =\xi^{\mu_{1}} \ldots \xi^{\mu_{n}} \nabla_{\left(\mu_{1}\right.} \ldots \nabla_{\left.\mu_{n}\right)} F(x)
\end{aligned}
$$

onde os parênteses nos índices representam simetrização total. Esta expressão permite a expansão de $F(C(\tau))$ em série de potências de $\tau$. O cálculo desta série em $\tau=1$ fornece a fórmula de Taylor covariante

$$
F(\gamma(x, \xi))=\sum_{n=0}^{\infty} \frac{\xi^{\mu_{1}} \ldots \xi^{\mu_{n}}}{n !} \nabla_{\left(\mu_{1}\right.} \ldots \nabla_{\left.\mu_{n}\right)} F(x)
$$

que é a generalização da fórmula usual $F(x+\xi)=e^{\xi \frac{\partial}{\partial x}} F(x)$. Todas as derivadas em relação a $\xi$ podem ser calculadas:

$$
\left.\frac{\partial}{\partial \xi^{\mu_{1}}} \ldots \frac{\partial}{\partial \xi^{\mu_{n}}} F(\gamma(x, \xi))\right|_{\xi=0}=\nabla_{\left(\mu_{1}\right.} \ldots \nabla_{\left.\mu_{n}\right)} F(x)
$$

Vamos exibir os três primeiros operadores $\nabla_{\mu_{1}} \ldots \nabla_{\mu_{n}}$ :

$$
\begin{aligned}
\nabla_{\mu_{1}} & =\partial_{\mu_{1}} \\
\nabla_{\mu_{1}} \nabla_{\mu_{2}} & =\left(\partial_{\mu_{1}} \delta_{\mu_{2}}^{\nu_{2}}-\Gamma_{\mu_{1} \mu_{2}}^{\nu_{2}}\right) \partial_{\nu_{2}} \\
\nabla_{\mu_{1}} \nabla_{\mu_{2}} \nabla_{\mu_{3}} & =\left(\partial_{\mu_{1}} \delta_{\mu_{2}}^{\nu_{2}} \delta_{\mu_{3}}^{\nu_{3}}-\Gamma_{\mu_{1} \mu_{2}}^{\nu_{2}} \delta_{\mu_{3}}^{\nu_{3}}-\Gamma_{\mu_{1} \mu_{3}}^{\nu_{3}} \delta_{\mu_{2}}^{\nu_{2}}\right)\left(\partial_{\nu_{2}} \delta_{\nu_{3}}^{\rho_{3}}-\Gamma_{\nu_{2} \nu_{3}}^{\rho_{3}}\right) \partial_{\rho_{3}}
\end{aligned}
$$

(Para uso em (C.20) e (C.21), os operadores (C.22, C.23, C.24, ...) ainda precisam ser simetrizados nos índices $\mu$.)

Uma conseqüência de (C.20), fazendo $F(x)=x^{\mu}$, é a expansão

$$
\begin{aligned}
\gamma^{\mu}(x, \xi) & =x^{\mu}+\xi^{\mu}-\frac{1}{2} \Gamma_{\mu_{1} \mu_{2}}^{\mu}(x) \xi^{\mu_{1}} \xi^{\mu_{2}}+\frac{1}{6} \Gamma_{\mu_{1} \mu_{2} \mu_{3}}^{\mu}(x) \xi^{\mu_{1}} \xi^{\mu_{2}} \xi^{\mu_{3}}+\ldots \\
\Gamma_{\mu_{1} \mu_{2} \mu_{3}}^{\mu} & =2 \Gamma_{\left(\mu_{1} \mu_{2}\right.}^{\nu} \Gamma_{\left.\mu_{3}\right) \nu}^{\mu}-\Gamma_{\left(\mu_{1} \mu_{2}, \mu_{3}\right)}^{\mu}
\end{aligned}
$$

Usando a fórmula (C.10), temos também a expansão

$$
\beta^{\mu}(x, \xi)=\xi^{\mu}-\Gamma_{\mu_{1} \mu_{2}}^{\mu}(x) \xi^{\mu_{1}} \xi^{\mu_{2}}+\frac{1}{2} \Gamma_{\mu_{1} \mu_{2} \mu_{3}}^{\mu}(x) \xi^{\mu_{1}} \xi^{\mu_{2}} \xi^{\mu_{3}}+\ldots
$$




\section{C.2 Coordenadas normais e geodésica mínima}

A equação (C.25) implica

$$
\left.\frac{\partial \gamma^{\mu}}{\partial \xi^{\nu}}\right|_{\xi=0}=\delta_{\nu}^{\mu}
$$

Esta matriz é inversível, e portanto, pelo teorema da função inversa, a função $\xi \longmapsto$ $\gamma\left(x_{0}, \xi\right)$, é um difeomorfismo em uma vizinhança de $\xi=0$, para qualquer ponto $x_{0}$. As novas coordenadas $x^{\prime}$ definidas por $x=\varphi^{-1}\left(x^{\prime}\right)=\gamma\left(x_{0}, x^{\prime}\right)$ são chamadas de coordenadas normais em torno do ponto $x_{0}{ }^{1}$. A equação (C.8) mostra que às coordenadas $x=x_{0}$ correspondem as coordenadas $x^{\prime}=0$. A propriedade (C.26) implica que $\frac{\partial x^{\mu}}{\partial x^{\prime \nu}}(0)=\delta_{\nu}^{\mu} \mathrm{e}$, portanto, a passagem às coordenadas normais não altera os tensores no ponto $x=x_{0}, x^{\prime}=0$.

As curvas $C^{\prime \mu}(\tau)=\tau \xi^{\mu}$ no sistema de coordenadas normais $x^{\prime}$ são mapeadas, no sistema de coordenadas $x$, nas curvas $C^{\mu}(\tau)=\gamma^{\mu}\left(x_{0}, \tau \xi\right)$, que são as geodésicas que passam pelo ponto $x_{0}$, de acordo com a equação (C.9). Isto significa que, no sistema de coordenadas $x^{\prime}$, as geodésicas que passam por $x^{\prime}=0$ são as linhas retas $C^{\prime \mu}(\tau)=\tau \xi^{\mu}$. Assim, pela mesma equação (C.9), a função exponencial, no sistema de coordenadas $x^{\prime}$ tem a propriedade

$$
\gamma^{\prime}(0, \xi)=\xi
$$

Pelo termo quadrático da expansão (C.25), vemos que, nas coordenadas normais, a conexão no ponto 0 é nula,

$$
\Gamma_{\mu_{1} \mu_{2}}^{\prime \mu}(0)=0
$$

Pelo termo cúbico, temos

$$
\begin{aligned}
0 & =\Gamma_{\left(\mu_{1} \mu_{2}, \mu_{3}\right)}^{\prime \mu}(0) \\
& =\frac{1}{6}\left(\Gamma_{\mu_{1} \mu_{2}, \mu_{3}}^{\prime \mu}+\Gamma_{\mu_{1} \mu_{3}, \mu_{2}}^{\prime \mu}+\Gamma_{\mu_{2} \mu_{1}, \mu_{3}}^{\prime \mu}+\Gamma_{\mu_{2} \mu_{3}, \mu_{1}}^{\prime \mu}+\Gamma_{\mu_{3} \mu_{1}, \mu_{2}}^{\prime \mu}+\Gamma_{\mu_{3} \mu_{2}, \mu_{1}}^{\prime \mu}\right)_{x^{\prime}=0}
\end{aligned}
$$

Contraindo os índices $\mu$ e $\mu_{3}$ e usando a simetria da conexão no índices inferiores, obtemos a identidade

$$
\Gamma_{\mu_{1} \mu_{2}, \mu}^{\prime \mu}(0)+\Gamma_{\mu_{1}, \mu_{2}}^{\prime}(0)+\Gamma_{\mu_{2}, \mu_{1}}^{\prime}(0)=0
$$

Pela fórmula (B.2), temos $\Gamma_{\mu_{1}, \mu_{2}}^{\prime}=\Gamma_{\mu_{2}, \mu_{1}}^{\prime}$. Assim, obtemos a identidade válida em

\footnotetext{
${ }^{1}$ As coordenadas normais em torno de um mesmo ponto não são únicas, mas dependem do sistema de coordenadas inicial $x$ onde se definiu a função exponencial $\gamma(x, \xi)$. Diferentes coordenadas normais em torno de um mesmo ponto se relacionam linearmente.
} 


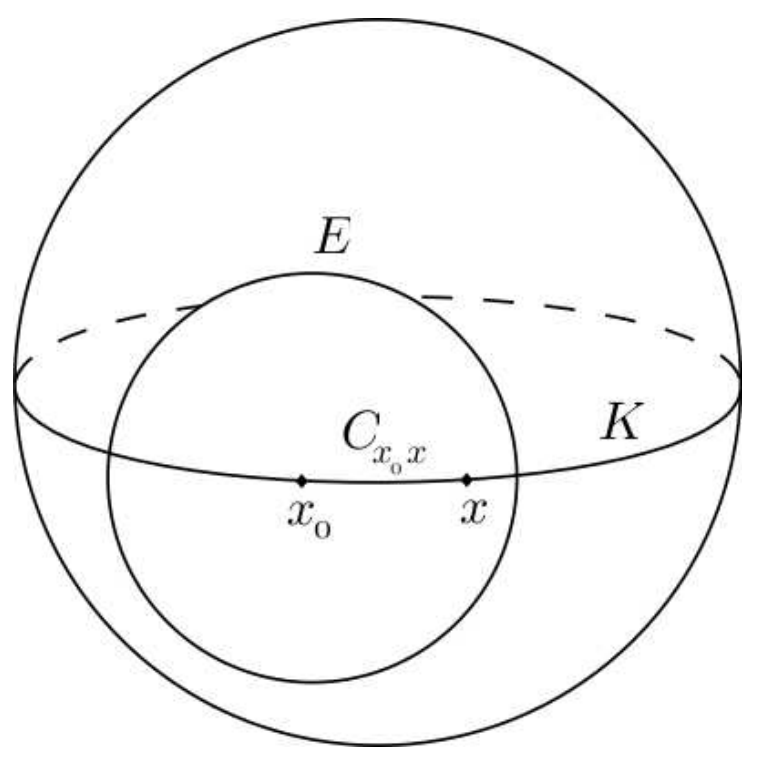

Figura C.2: Geodésica mínima $C_{x_{0} x}$ e não-mínima $K$ em uma esfera.

coordenadas normais

$$
\Gamma_{\mu_{1} \mu_{2}, \mu}^{\prime \mu}(0)+2 \Gamma_{\mu_{1}, \mu_{2}}^{\prime}(0)=0 .
$$

Usando as fórmulas (C.28) e (C.29), podemos exprimir o tensor de Ricci como

$$
R_{\mu \nu}^{\prime}(0)=\Gamma_{\mu \nu, \rho}^{\prime \rho}(0)-\Gamma_{\mu, \nu}^{\prime}(0)=-3 \Gamma_{\mu, \nu}^{\prime}(0)=\frac{3}{2} \Gamma_{\mu \nu, \rho}^{\rho}(0)
$$

Dados dois pontos $x_{0}$ e $x$, pode-se perguntar se existe uma geodésica $C(\tau)$ que os une, isto é, $C(0)=x_{0}, C(1)=x$. Esta geodésica certamente existe se $x$ está na região em que $\gamma\left(x_{0}, \xi\right)$ é um difeomorfismo, pois se $x=\gamma\left(x_{0}, \xi\right)$, então $C(\tau)=$ $\gamma\left(x_{0}, \tau \xi\right)$ é a geodésica que procuramos (ver a equação (C.9)). Em geral, existirá mais de uma geodésica unindo dois pontos quaisquer (em uma esfera, por exemplo, sempre existem duas geodésicas). Podemos, no entanto, definir uma geodésica de características únicas chamada geodésica mínima através do procedimento descrito a seguir.

Consideremos uma vizinhança de um ponto $x_{0}$ em que a função $\xi \longmapsto \gamma\left(x_{0}, \xi\right)$ seja um difeomorfismo, e definamos o difeomorfismo $\xi=\varphi(x)$ por $\varphi^{-1}(\xi)=\gamma\left(x_{0}, \xi\right)$ nesta região. Consideremos uma esfera $D$-dimensional $E^{\prime}=\left\{\xi \in \mathbb{R}^{D} \mid \xi^{2}=g_{\mu \nu}\left(x_{0}\right) \xi^{\mu} \xi^{\nu}<r^{2}\right\}$ inteiramente contida na vizinhança em que $\xi \longmapsto \gamma\left(x_{0}, \xi\right)$ é um difeomorfismo (esta esfera existe com $r>0$, já que a vizinhança de $x_{0}$ é um conjunto aberto) e definamos o conjunto $E=\varphi^{-1}\left(E^{\prime}\right)$, que também é aberto, pois $\varphi$ é um difeomorfismo. A função $\xi \longmapsto \gamma\left(x_{0}, \xi\right)$ é um difeomorfismo em $E^{\prime}$, logo para quanquer ponto $x \in E$ 
existe um único $\xi \in E^{\prime}$ tal que $x=\gamma\left(x_{0}, \xi\right)$. Definimos então a geodésica

$$
C_{x_{0} x}(\tau)=\gamma\left(x_{0}, \tau \xi\right)
$$

que une $x_{0}$ a $x$. Esta geodésica é chamada de geodésica mínima que une $x_{0}$ a $x$, e está definida apenas quando $x \in E$ como construído acima. Seu comprimento (C.2) é $S\left[C_{x_{0}, x}\right]=\sqrt{g_{\mu \nu}\left(x_{0}\right) \xi^{\mu} \xi^{\nu}}<r$. Consideremos, se houver, alguma outra geodésica $K(\tau)$ diferente de $C_{x_{0} x}$ que una $x_{0}$ a $x$, isto é, $K(0)=x_{0}$ e $K(1)=x$. Pela definição da função exponencial, $x=\gamma\left(x_{0}, \xi_{K}\right)$, com $\xi_{K}=\dot{K}(0)$. Como a função $\xi \longmapsto \gamma\left(x_{0}, \xi\right)$ é um difeomorfismo, e portanto biunívoca em $E^{\prime}$, vemos que $\xi_{K} \notin E^{\prime}$, $\operatorname{logo} S[K] \geq r$. Logo, a geodésica mínima é a geodésica de menor comprimento que une os dois pontos $x_{0}$ e $x$. O argumento

$$
\tau_{f}=\frac{r}{\sqrt{g_{\mu \nu}\left(x_{0}\right) \xi_{K}^{\mu} \xi_{K}^{\nu}}}
$$

é tal que $0<\tau_{f} \leq 1, \operatorname{logo} K\left(\tau_{f}\right)=\gamma\left(x_{0}, \tau_{f} \xi_{K}\right)$ é um ponto da geodésica. Temos

$$
\sqrt{g_{\mu \nu}\left(x_{0}\right)\left(\tau_{f} \xi_{K}\right)^{\mu}\left(\tau_{f} \xi_{K}\right)^{\nu}}=r
$$

$\operatorname{logo} \tau_{f} \xi_{K} \notin E^{\prime}$ e $\tau_{f} \xi_{K} \in \overline{E^{\prime}}$, onde $\bar{A}$ representa o fecho (no sentido topológico) do conjunto $A$. Como $\xi \longmapsto \gamma\left(x_{0}, \xi\right)$ é um difeomorfismo, $K\left(\tau_{f}\right)=\gamma\left(x_{0}, \tau_{f} \xi_{K}\right) \notin E$ e $K\left(\tau_{f}\right) \in \bar{E}$. Assim, A geodésica mínima é a única inteiramente contida em $E$. Finalmente, como $\varphi$ é um difeomorfismo, $\lim _{x \rightarrow x_{0}} \xi=0$, e, portanto

$$
\lim _{x \rightarrow x_{0}} S\left[C_{x_{0} x}\right]=0
$$

Como qualquer outra geodésica $K$ que una $x_{0}$ a $x$ obedece a $S[K] \geq r$, vemos que a geodésica mínima é a única que apresenta o comportamento limite acima. A situação geral deste teorema está reresentada na figura C.2 quando o espaço de Riemann é uma esfera.

\section{C.3 Determinante jacobiano do fluxo geodésico}

A equação diferencial (C.1) decorre da lagrangiana não singular

$$
L(x, \dot{x})=\frac{1}{2} g_{\mu \nu}(x) \dot{x}^{\mu} \dot{x}^{\nu}
$$


A passagem ao espaço de fase canônico se dá, portanto, com a transformação

$$
p_{\mu}=\frac{\partial L}{\partial \dot{x}^{\mu}}=g_{\mu \nu}(x) \dot{x}^{\nu} .
$$

Podemos definir uma função $G: \mathbb{R}^{2 D} \rightarrow \mathbb{R}^{2 D}$ que transforma um ponto $(x, \xi)$ do espaço de fase não canônico em um ponto $(x, p)$ do espaço de fase canônico por

$$
G(x, \xi)=\left(\left(x^{\mu}\right),\left(g_{\mu \nu}(x) \xi^{\nu}\right)\right)
$$

cuja inversa é

$$
G^{-1}(x, p)=\left(\left(x^{\mu}\right),\left(g^{\mu \nu}(x) p_{\nu}\right)\right)
$$

Desta maneira, uma trajetória $(C(\tau), P(\tau))$ no espaço de fase canônico é dada em função de uma trajetória $(C(\tau), \dot{C}(\tau))$ no espaço de fase não canônico por $(C(\tau), P(\tau))=G(C(\tau), \dot{C}(\tau))$. Usando o fluxo geodésico (C.13), $(C(\tau), P(\tau))=$ $G\left(T_{\tau}(x, \xi)\right)$, onde $x=C(0)$ e $\xi=\dot{C}(0)$ são as condições iniciais no espaço de fase não canônico. Estas condições iniciais podem, por sua vez, ser transportadas ao espaço de fase canônico por $(x, p)=G^{-1}(x, \xi)$, de modo que $p=\dot{P}(0)$ é a condição inicial da variável de momento. Assim, $(C(\tau), P(\tau))=G\left(T_{\tau}\left(G^{-1}(x, p)\right)\right)$, o que define o fluxo geodésico canônico

$$
T_{\tau}^{\mathrm{C}}(x, p)=G\left(T_{\tau}\left(G^{-1}(x, p)\right)\right)
$$

Pelo teorema de Liouville, o fluxo canônico tem determinante jacobiano igual a 1. Isto permite calcular o determinante jacobiano do fluxo geodésico por

$$
1=\frac{\partial\left(T_{\tau}^{\mathrm{C}}\right)}{\partial(x, p)}=\frac{\partial(G)}{\partial(x, \xi)}\left(T_{\tau}(x, \xi)\right) \frac{\partial\left(T_{\tau}\right)}{\partial(x, \xi)}(x, \xi) \frac{\partial\left(G^{-1}\right)}{\partial(x, p)}(x, p)
$$

Os determinantes jacobianos de $G$ e $G^{-1}$ são dados por

$$
\begin{gathered}
\frac{\partial(G)}{\partial(x, \xi)}(x, \xi)=\operatorname{det}\left(\begin{array}{cc}
\frac{\partial x^{\alpha}}{\partial x^{\beta}} & \frac{\partial x^{\alpha}}{\partial \xi^{\beta}} \\
\frac{\partial g_{\alpha \nu}}{\partial x^{\beta}} \xi^{\nu} & g_{\alpha \nu} \frac{\partial \xi^{\nu}}{\partial \xi^{\beta}}
\end{array}\right)=\operatorname{det}\left(\begin{array}{cc}
\delta_{\beta}^{\alpha} & 0 \\
\frac{\partial g_{\alpha \nu}}{\partial x^{\beta}} & g_{\alpha \beta}
\end{array}\right)=g(x) \\
\frac{\partial\left(G^{-1}\right)}{\partial(x, p)}(x, p)=\operatorname{det}\left(\begin{array}{cc}
\frac{\partial x^{\alpha}}{\partial x^{\beta}} & \frac{\partial x^{\alpha}}{\partial p_{\beta}} \\
\frac{\partial g^{\alpha \nu}}{\partial x^{\beta}} p_{\nu} & g^{\alpha \nu} \frac{\partial p_{\nu}}{\partial p_{\beta}}
\end{array}\right)=\operatorname{det}\left(\begin{array}{cc}
\delta_{\beta}^{\alpha} & 0 \\
\frac{\partial g^{\alpha \nu}}{\partial x^{\beta}} p_{\nu} & g^{\alpha \beta}
\end{array}\right)=g^{-1}(x) .
\end{gathered}
$$

Desta maneira, temos

$$
\frac{\partial\left(T_{\tau}\right)}{\partial(x, \xi)}(x, \xi)=\frac{g(x)}{g(\gamma(x, \tau \xi))} .
$$




\section{Apêndice D}

\section{Uma fórmula para a derivada do produto}

Sejam $f$ e $g$ duas funções quaisquer e $T^{\mu_{1} \cdots \mu_{n}}$ coeficientes totalmente simétricos nos índices $\mu_{1} \cdots \mu_{n}$. Vamos provar por indução a seguinte proposição útil ${ }^{1}$ :

$$
T^{\mu_{1} \cdots \mu_{n}} \partial_{\mu_{1}} \cdots \partial_{\mu_{n}} f g=T^{\mu_{1} \cdots \mu_{n}} \sum_{k=0}^{n}\left(\begin{array}{l}
n \\
k
\end{array}\right)\left(\partial_{\mu_{1}} \cdots \partial_{\mu_{k}} f\right)\left(\partial_{\mu_{k+1}} \cdots \partial_{\mu_{n}} g\right)
$$

onde

$$
\left(\begin{array}{l}
n \\
k
\end{array}\right)=\frac{n !}{k !(n-k) !}
$$

são os coeficientes binomiais. Se $n=1$, a proposição é claramente verdadeira: $T^{\mu} \partial_{\mu} f g=T^{\mu}\left(\left(\partial_{\mu} f\right) g+f\left(\partial_{\mu} g\right)\right)$. Suponhamos que ela seja verdadeira para $n$.

$$
\begin{aligned}
& T^{\mu_{1} \cdots \mu_{n} \mu_{n+1}} \partial_{\mu_{1}} \cdots \partial_{\mu_{n}} \partial_{\mu_{n+1}} f g \\
& =T^{\mu_{1} \cdots \mu_{n} \mu_{n+1}} \partial_{\mu_{1}} \cdots \partial_{\mu_{n}}\left[\left(\partial_{\mu_{n+1}} f\right) g+g\left(\partial_{\mu_{n+1}} f\right)\right] .
\end{aligned}
$$

${ }^{1}$ Esta fórmula é uma generalização multi-dimensional da fórmula de Leibnitz

$$
\frac{d^{n}}{d x^{n}}(f g)=\sum_{k=0}^{n}\left(\begin{array}{l}
n \\
k
\end{array}\right) \frac{d^{k} f}{d x^{k}} \frac{d^{n-k} g}{d x^{n-k}}
$$


$T^{\mu_{1} \cdots \mu_{n} \mu_{n+1}}$ é simétrico em todos os índices e portanto simétrico no subconjunto $\mu_{1} \cdots \mu_{n}$. Aplicando então a hipótese de indução, a quantidade acima torna-se

$$
\begin{gathered}
T^{\mu_{1} \cdots \mu_{n} \mu_{n+1}}\left(\sum_{k=0}^{n}\left(\begin{array}{l}
n \\
k
\end{array}\right)\left(\partial_{\mu_{1}} \cdots \partial_{\mu_{k}} \partial_{\mu_{n+1}} f\right)\left(\partial_{\mu_{k+1}} \cdots \partial_{\mu_{n}} g\right)\right. \\
\left.+\sum_{k=0}^{n}\left(\begin{array}{l}
n \\
k
\end{array}\right)\left(\partial_{\mu_{1}} \cdots \partial_{\mu_{k}} f\right)\left(\partial_{\mu_{k+1}} \cdots \partial_{\mu_{n}} \partial_{\mu_{n+1}} g\right)\right)
\end{gathered}
$$

Devido à simetria total de $T^{\mu_{1} \cdots \mu_{n} \mu_{n+1}}$, a primeira parcela acima se torna

$$
\begin{aligned}
& T^{\mu_{1} \cdots \mu_{n} \mu_{n+1}} \sum_{k=0}^{n}\left(\begin{array}{l}
n \\
k
\end{array}\right)\left(\partial_{\mu_{1}} \cdots \partial_{\mu_{k}} \partial_{\mu_{n+1}} f\right)\left(\partial_{\mu_{k+1}} \cdots \partial_{\mu_{n}} g\right) \\
& =T^{\mu_{1} \cdots \mu_{n} \mu_{n+1}} \sum_{k=0}^{n}\left(\begin{array}{l}
n \\
k
\end{array}\right)\left(\partial_{\mu_{1}} \cdots \partial_{\mu_{k}} \partial_{\mu_{k+1}} f\right)\left(\partial_{\mu_{k+2}} \cdots \partial_{\mu_{n+1}} g\right) \\
& =T^{\mu_{1} \cdots \mu_{n+1}} \sum_{k=1}^{n+1}\left(\begin{array}{c}
n \\
k-1
\end{array}\right)\left(\partial_{\mu_{1}} \cdots \partial_{\mu_{k}} f\right)\left(\partial_{\mu_{k+1}} \cdots \partial_{\mu_{n+1}} g\right) .
\end{aligned}
$$

Substituindo em (D.2),

$$
\begin{aligned}
& T^{\mu_{1} \cdots \mu_{n} \mu_{n+1}} \partial_{\mu_{1}} \cdots \partial_{\mu_{n}} \partial_{\mu_{n+1}} f g \\
& =T^{\mu_{1} \cdots \mu_{n} \mu_{n+1}}\left(\sum_{k=1}^{n}\left(\left(\begin{array}{c}
n \\
k
\end{array}\right)+\left(\begin{array}{c}
n \\
k-1
\end{array}\right)\right)\left(\partial_{\mu_{1}} \cdots \partial_{\mu_{k}} f\right)\left(\partial_{\mu_{k+1}} \cdots \partial_{\mu_{n+1}} g\right)\right. \\
& \left.\quad+\left(\begin{array}{c}
n \\
0
\end{array}\right)\left(\partial_{\mu_{1}} \cdots \partial_{\mu_{n+1}} f\right) g+\left(\begin{array}{l}
n \\
n
\end{array}\right) f\left(\partial_{\mu_{1}} \cdots \partial_{\mu_{n+1}} g\right)\right) .
\end{aligned}
$$

Usando a propriedade dos coeficientes binomiais $\left(\begin{array}{l}n \\ k\end{array}\right)+\left(\begin{array}{c}n \\ k-1\end{array}\right)=\left(\begin{array}{c}n+1 \\ k\end{array}\right),\left(\begin{array}{c}n+1 \\ 0\end{array}\right)=\left(\begin{array}{l}n \\ 0\end{array}\right)=$ $\left(\begin{array}{l}n+1 \\ n+1\end{array}\right)=\left(\begin{array}{l}n \\ n\end{array}\right)=1$ chegamos à fórmula (D.1), que queríamos demonstrar. 


\section{Referências Bibliográficas}

[1] Langouche, F., Roekaerts, D., Tirapegui, E., Functional Integration and Semiclassical Expansion (Reidel, Dordrecht, 1982)

[2] Emmrich C., Commun. Math. Phys. 151515 (1993)

[3] Plebanski J. F., Przanowski M., Tosiek J. Acta Phys. Polon. B27 1961 (1996)

[4] Plebanski, J. F., Przanowski, M., Turrubiates, F. J., Journal of Physics A33, Issue 4, pp. 795-804 (2000).

[5] J.L.M. Assirati, Generalização covariante da correspondência de Weyl e quantização da partícula no espaço curvo, Dissertação (Mestrado) IFUSP (2001)

[6] B.S. DeWitt, Phys. Rev. 85 (1952), 653

[7] B. S. DeWitt, Rev. Mod. Phys. 29 (1957), 377

[8] R. Penrose, W. Rindler, Spinors and space-time (1986)

[9] F. A. Berezin and M. A. Shubin, The Schrödinger Equation (Kluwer Academic Publishers, Dordrecht, 1991)

[10] Berezin, F. A., Theor. Math. Phys. 6, 141 (1971) 